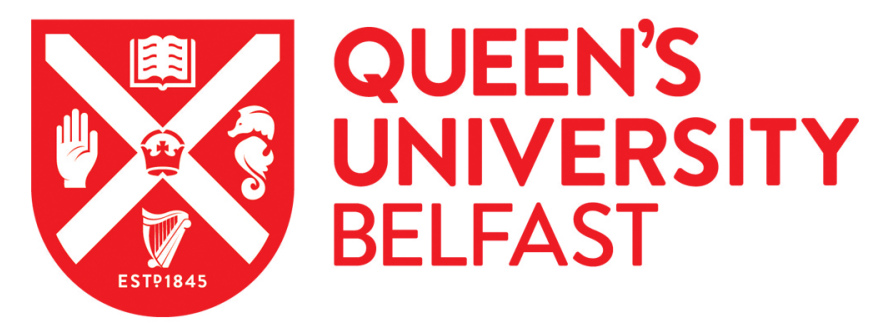

\title{
Cosmological Constraints from Measurements of Type la Supernovae Discovered During the First 1.5 Yr of the Pan-STARRS1 Survey
}

Rest, A., Scolnic, D., Foley, R. J., Huber, M. E., Chornock, R., Narayan, G., Tonry, J. L., Berger, E., Soderberg, A. M., Stubbs, C. W., Riess, A., Kirshner, R. P., Smartt, S. J., Schlafly, E., Rodney, S., Botticella, M. T., Brout, D., Challis, P., Czekala, I., ... Waters, C. (2014). Cosmological Constraints from Measurements of Type la Supernovae Discovered During the First 1.5 Yr of the Pan-STARRS1 Survey. Astrophysical Journal, 795(1), [44]. https://doi.org/10.1088/0004-637X/795/1/44

Published in:

Astrophysical Journal

Document Version:

Publisher's PDF, also known as Version of record

Queen's University Belfast - Research Portal:

Link to publication record in Queen's University Belfast Research Portal

Publisher rights

(C) 2014 The American Astronomical Society. All rights reserved.

General rights

Copyright for the publications made accessible via the Queen's University Belfast Research Portal is retained by the author(s) and / or other copyright owners and it is a condition of accessing these publications that users recognise and abide by the legal requirements associated with these rights.

Take down policy

The Research Portal is Queen's institutional repository that provides access to Queen's research output. Every effort has been made to ensure that content in the Research Portal does not infringe any person's rights, or applicable UK laws. If you discover content in the Research Portal that you believe breaches copyright or violates any law, please contact openaccess@qub.ac.uk. 


\title{
COSMOLOGICAL CONSTRAINTS FROM MEASUREMENTS OF TYPE Ia SUPERNOVAE DISCOVERED DURING THE FIRST 1.5 yr OF THE Pan-STARRS1 SURVEY
}

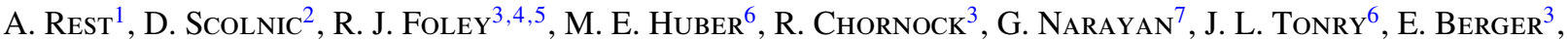 \\ A. M. Soderberg ${ }^{3}$, C. W. Stubbs ${ }^{3,7}$, A. Riess ${ }^{2}$, R. P. Kirshner ${ }^{3,7}$, S. J. SmartT ${ }^{8}$, E. Schlafly ${ }^{9}$, S. Rodney ${ }^{2,20}$, \\ M. T. Botticella ${ }^{10}$, D. Brout ${ }^{2}$, P. Challis ${ }^{3}$, I. CzeKala ${ }^{3}$, M. Drout ${ }^{3}$, M. J. Hudson ${ }^{11,12}$, R. KotaK ${ }^{8}$, C. LeibleR $^{13}$, \\ R. Lunnan ${ }^{3}$, G. H. Marion ${ }^{3}$, M. McCrum ${ }^{8}$, D. Milisavljevic ${ }^{3}$, A. Pastorello ${ }^{14}$, N. E. Sanders ${ }^{3}$, K. Smith ${ }^{8}$, E. Stafford ${ }^{2}$, \\ D. Thilker ${ }^{2}$, S. Valenti ${ }^{15,16}$, W. M. Wood-Vasey ${ }^{17}$, Z. Zheng ${ }^{2}$, W. S. BurgetT ${ }^{6}$, K. C. Chambers ${ }^{6}$, L. Denneau ${ }^{6}$, \\ P. W. Draper ${ }^{18}$, H. Flewelling ${ }^{6}$, K. W. Hodapp ${ }^{6}$, N. Kaiser ${ }^{6}$, R.-P. Kudritzki ${ }^{6}$, E. A. Magnier ${ }^{6}$, N. Metcalfe ${ }^{18}$, \\ P. A. Price ${ }^{19}$, W. Sweeney ${ }^{6}$, R. Wainscoat ${ }^{6}$, AND C. Waters ${ }^{6}$ \\ ${ }^{1}$ Space Telescope Science Institute, 3700 San Martin Drive, Baltimore, MD 21218, USA \\ 2 Department of Physics and Astronomy, Johns Hopkins University, 3400 North Charles Street, Baltimore, MD 21218, USA \\ ${ }^{3}$ Harvard-Smithsonian Center for Astrophysics, 60 Garden Street, Cambridge, MA 02138, USA \\ ${ }^{4}$ Astronomy Department, University of Illinois at Urbana-Champaign, 1002 West Green Street, Urbana, IL 61801, USA \\ ${ }^{5}$ Department of Physics, University of Illinois Urbana-Champaign, 1110 West Green Street, Urbana, IL 61801, USA \\ ${ }^{6}$ Institute for Astronomy, University of Hawaii, 2680 Woodlawn Drive, Honolulu, HI 96822, USA \\ ${ }^{7}$ Department of Physics, Harvard University, 17 Oxford Street, Cambridge, MA 02138, USA \\ ${ }^{8}$ Astrophysics Research Centre, School of Mathematics and Physics, Queens University Belfast, Belfast BT71NN, UK \\ ${ }^{9}$ Max Planck Institute for Astronomy, Königstuhl 17, D-69117 Heidelberg, Germany \\ ${ }^{10}$ INAF-Osservatorio Astronomico di Capodimonte, Salita Moiariello 16, I-80131 Napoli, Italy \\ ${ }^{11}$ University of Waterloo, 200 University Avenue West, Waterloo, ON N2L 3G1, Canada \\ ${ }^{12}$ Perimeter Institute for Theoretical Physics, 31 Caroline Street North, Waterloo, Ontario, N2L 2Y5, Canada \\ ${ }^{13}$ Department of Astronomy \& Astrophysics, University of California, Santa Cruz, CA 95060, USA \\ ${ }^{14}$ INAF-Osservatorio Astronomico di Padova, Vicolo dell'Osservatorio 5, I-35122 Padova, Italy \\ ${ }^{15}$ Las Cumbres Observatory Global Telescope Network, Inc., Santa Barbara, CA 93117, USA \\ ${ }^{16}$ Department of Physics, University of California Santa Barbara, Santa Barbara, CA 93106-9530, USA \\ ${ }_{17}$ PITT PACC, Department of Physics and Astronomy, University of Pittsburgh, Pittsburgh, PA 15260, USA \\ ${ }^{18}$ Department of Physics, University of Durham Science Laboratories, South Road, Durham DH1 3LE, UK \\ ${ }^{19}$ Department of Astrophysical Sciences, Princeton University, Princeton, NJ 08544, USA \\ Received 2013 October 17; accepted 2014 July 30; published 2014 October 13
}

\begin{abstract}
We present griz $_{\mathrm{P} 1}$ light curves of 146 spectroscopically confirmed Type Ia supernovae (SNe Ia; $0.03<z<0.65$ ) discovered during the first $1.5 \mathrm{yr}$ of the Pan-STARRS1 Medium Deep Survey. The Pan-STARRS1 natural photometric system is determined by a combination of on-site measurements of the instrument response function and observations of spectrophotometric standard stars. We find that the systematic uncertainties in the photometric system are currently $1.2 \%$ without accounting for the uncertainty in the Hubble Space Telescope Calspec definition of the AB system. A Hubble diagram is constructed with a subset of 113 out of $146 \mathrm{SNe}$ Ia that pass our light curve quality cuts. The cosmological fit to 310 SNe Ia (113 PS1 SNe Ia + 222 light curves from 197 low- $z \mathrm{SNe}$ Ia), using only supernovae ( $\mathrm{SNe}$ ) and assuming a constant dark energy equation of state and flatness, yields $w=-1.120_{-0.206}^{+0.360}(\text { Stat })_{-0.291}^{+0.269}$ (Sys). When combined with $\mathrm{BAO}+\mathrm{CMB}($ Planck $)+H_{0}$, the analysis yields $\Omega_{\mathrm{M}}=0.280_{-0.012}^{+0.013}$ and $w=-1.166_{-0.069}^{+0.072}$ including all identified systematics. The value of $w$ is inconsistent with the cosmological constant value of -1 at the $2.3 \sigma$ level. Tension endures after removing either the baryon acoustic oscillation (BAO) or the $H_{0}$ constraint, though it is strongest when including the $H_{0}$ constraint. If we include WMAP9 cosmic microwave background (CMB) constraints instead of those from Planck, we find $w=-1.124_{-0.065}^{+0.083}$, which diminishes the discord to $<2 \sigma$. We cannot conclude whether the tension with flat $\Lambda$ CDM is a feature of dark energy, new physics, or a combination of chance and systematic errors. The full Pan-STARRS1 SN sample with $\sim$ three times as many $\mathrm{SNe}$ should provide more conclusive results.
\end{abstract}

Key words: cosmological parameters - cosmology: observations - dark energy - supernovae: general

Online-only material: color figures, machine-readable table

\section{INTRODUCTION}

We have used the medium deep field (MDF) survey of the Pan-STARRS1 (PS1) Science program as the source for detecting thousands of transient events. These include supernovae (SNe) and other transients of unusual types (Botticella et al. 2010; Gezari et al. 2010, 2012; Chomiuk et al. 2011; Narayan et al. 2011; Berger et al. 2012, 2013; Lee et al. 2012; Sanders

\footnotetext{
${ }^{20}$ Hubble Postdoctoral Fellow.
}

et al. 2012, 2013; Chornock et al. 2013; Lunnan et al. 2013). In parallel, we are also exploiting the PS1 $3 \pi$ survey for detection of brighter transients at lower redshifts (Pastorello et al. 2010; Valenti et al. 2012; Inserra et al. 2013). Here, we describe 146 Type Ia supernovae ( $\mathrm{SNe}$ Ia) from the first year and a half of observations that we use to measure the history of cosmic expansion to help constrain the properties of dark energy. This report explains our observing strategy, photometric reductions, and cosmological analysis. The systematic errors in the photometry are discussed at length in this paper, while the companion 
paper by Scolnic et al. (2014b, hereafter S14) focuses on the systematic uncertainties in the cosmological analysis.

$\mathrm{SNe}$ Ia have been proven to be reliable standard candles at cosmological distances. The Supernova Cosmology Project and the High-Z Supernova Team discovered SNe Ia at redshifts from 0.3 to 1 that provided the first evidence for cosmic acceleration (Riess et al. 1998; Perlmutter et al. 1999). This result in combination with measurements of the baryon acoustic peak in the large-scale correlation function of galaxies (e.g., Blake et al. 2011; Anderson et al. 2012) and the power spectrum of fluctuations in the cosmic microwave background (CMB; e.g., Hinshaw et al. 2013; Planck Collaboration et al. 2013) indicates that we live in a flat, accelerating universe composed of baryons, dark matter, and dark energy.

SNe Ia are more-or-less homogeneous thermonuclear explosions of white dwarfs. The discovery by Phillips (1993) that the shape of the SN light curve is related to the SN Ia luminosity opened the door for their use as precise cosmic standard candles. In 1996, the Calán/Tololo group published light curves of 29 $\mathrm{SNe}$ in four colors (Hamuy et al. 1996a). This data set was large enough to develop reliable ways to use the SNe light curves to determine the intrinsic luminosity of SNe Ia and to measure the luminosity distance with an precision of $\sim 10 \%$ to each object (e.g., $\Delta m_{15}$; Hamuy et al. 1996b, 1996c; Phillips et al. 1999, and MLCS, Riess et al. 1996). Since then, new approaches and new algorithms have improved light curve fitting so that wellobserved SNe Ia have individual luminosity distances good to 5\% (e.g., MLCS2K2, Jha et al. 2007; Stretch, Goldhaber et al. 2001; SALT, Guy et al. 2007; SiFTO, Conley et al. 2008; and BayeSN, Mandel et al. 2009, 2011).

With more accurate methods to determine distances of SNe Ia, it is now possible to probe the nature of dark energy by constraining its equation of state, characterized by the parameter $w=P /\left(\rho c^{2}\right)$, where $P$ is its pressure and $\rho$ is its density. Evidence for cosmic acceleration and constraints on the dark energy are derived from the combination of low-redshift samples and high-redshift samples. In the last decade, many groups have worked on assembling large sets of low-redshift SNe (e.g., CfA1-CfA4, Riess et al. 1999; Jha et al. 2006; Hicken et al. 2009a, 2009b, 2012; CSP, Contreras et al. 2010; Folatelli et al. 2010; Stritzinger et al. 2011; LOSS, Ganeshalingam et al. 2013). In total, the currently published low- $z$ SNe sample comprises more than 500 SNe Ia. There have been three main surveys probing the higher redshift range, ESSENCE (Miknaitis et al. 2007; Wood-Vasey et al. 2007), SNLS (Conley et al. 2011; Sullivan et al. 2011), and SDSS (Frieman et al. 2008; Kessler et al. 2009a). These surveys have overlapping redshift ranges from $0.1 \lesssim z \lesssim 0.4$ for SDSS, $0.2 \lesssim z \lesssim 0.7$ for ESSENCE, and $0.3 \lesssim z \lesssim 1.1$ for SNLS. For a more complete review of SNe Ia cosmology from these surveys, see Kirshner (2010).

Increasing the number of $\mathrm{SNe}$ alone will not improve the limits on $w$ unless we decrease the systematic errors as well. The photometric calibration is currently the most significant source of systematic bias (Sullivan et al. 2011), since different sets of SNe with light curves from different telescope/detector systems are compared to each other. There are significant efforts underway to improve and homogenize the photometric calibration of previous (e.g., SNLS and SDSS; Betoule et al. 2013) and ongoing wide-field transient surveys like PS1 (Kaiser et al. 2010), the Palomar Transient Factory (PTF; Rau et al. 2009), and the Dark Energy Survey (Flaugher et al. 2012). Another technical issue is how the light curve fitters deal with the degeneracy between intrinsic luminosity, extinction, and intrinsic color. For example, Scolnic et al. (2014a) show that the distance residuals can be decreased by assuming an intrinsic SN Ia color dispersion as a prior, which also is consistent with a Milky Way-like reddening law.

In the future, the full PS1 SN sample will be uniquely suited to better constrain the nature of dark energy by covering a very wide redshift range $(0.03<z<0.65)$ with a single instrument. Although the PS1 sample presented in this paper does not have enough low-redshift $\mathrm{SNe}$ Ia for a stand-alone cosmological analysis, it is sufficient for us to investigate the best approach to combining data from separate photometric systems. In this paper, we use the $146 \mathrm{SNe}$ Ia from the first $1.5 \mathrm{yr}$ (2009 September to 2011 May) of PS1 to constrain the cosmological parameters along with a joint constraint by other cosmological probes. We emphasize the reduction of systematic uncertainties that affect the measurement of dark energy properties. The number of SNe Ia that pass all light-curve quality cuts (113) is almost half of the largest published individual high-redshift sample (248; Conley et al. 2011; Sullivan et al. 2011). Our sample allows us to identify where we have the largest systematic errors and to develop remedies for them. Future analysis of the full data set will build on our current analysis, and improve today's largest systematic uncertainties: the photometric calibration, and flaws in light curve fitting techniques.

This paper is organized as follows. In Section 2, we introduce the technical aspects of the PS1 system. We describe the transient alert system and the spectroscopic follow-up in Sections 3 and 4, respectively. The SNe Ia light curves are presented in Section 5, and the photometric calibration is presented in Section 6. We discuss how we determine distances from the SNe Ia light curves in Section 7. In Section 8, we determine the cosmological parameters by combining our sample of 113 high-quality SNe Ia with the low-redshift sample and constraints from other cosmological probes.

\section{Pan-STARRS1 SURVEY}

The PS1 system is a high-etendue wide-field imaging system designed for dedicated survey observations. The system is installed on the peak of Haleakala on the island of Maui in the Hawaiian island chain. We provide below a terse summary of the PS1 survey instrumentation. A more complete description of the PS1 system, both hardware and software, is provided by Kaiser et al. (2010).

The PS1 optical design (Hodapp et al. 2004) uses a $1.8 \mathrm{~m}$ diameter $f / 4.4$ primary mirror and a $0.9 \mathrm{~m}$ secondary. The telescope delivers images with low distortion over a field diameter of $3.3 \mathrm{deg}$. An individual CCD cell has $800 \times$ 800 pixels, with $10 \mu \mathrm{m}$ pixels that subtend 0.258 arcsec. 64 of these CCD cells are grouped into an $8 \times 8$ array. The focal plane consists of 60 of these independent arrays, for a total of 1.4 gigapixel. The detectors are back-illuminated CCDs manufactured by Lincoln Laboratory, which are read out using a StarGrasp CCD controller in $7 \mathrm{~s}$ for a full unbinned image. Initial performance assessments are presented in Onaka et al. (2008).

The PS1 observations are obtained through a set of five broadband filters, which we have designated as $g_{\mathrm{P} 1}, r_{\mathrm{P} 1}, i_{\mathrm{P} 1}$, $z_{\mathrm{P} 1}$, and $y_{\mathrm{P} 1}\left(\right.$ grizy $\left.y_{\mathrm{P} 1}\right)$. These filters are similar to the ones used in SDSS, with the most significant difference in the $g$ band. We use the instrumental response functions determined by Tonry et al. (2012b).

In addition to covering the entire sky at $\delta>-30 \mathrm{deg}$ in five bands $(3 \pi$ survey), the PS1 survey has obtained deeper, higher 
Table 1

Pan-STARRS1 MDFs

\begin{tabular}{lccccccc}
\hline \hline Field & R.A. & Decl. & $N_{\text {SN }}$ & \multicolumn{4}{c}{ FWHM in arcsec } \\
\cline { 5 - 8 } & $(\mathrm{J} 2000)$ & $(\mathrm{J} 2000)$ & & $g_{\mathrm{P} 1}$ & $r_{\mathrm{P} 1}$ & $i_{\mathrm{P} 1}$ & $z_{\mathrm{P} 1}$ \\
\hline MD01 & 035.875 & -04.250 & 15 & 1.25 & 1.15 & 1.05 & 1.03 \\
MD02 & 053.100 & -27.800 & 16 & 1.31 & 1.20 & 1.11 & 1.06 \\
MD03 & 130.592 & +44.317 & 20 & 1.18 & 1.09 & 1.06 & 1.03 \\
MD04 & 150.000 & +02.200 & 22 & 1.17 & 1.09 & 1.07 & 1.03 \\
MD05 & 161.917 & +58.083 & 13 & 1.24 & 1.17 & 1.06 & 0.99 \\
MD06 & 185.000 & +47.117 & 15 & 1.25 & 1.18 & 1.14 & 1.05 \\
MD07 & 213.704 & +53.083 & 15 & 1.23 & 1.13 & 1.14 & 1.08 \\
MD08 & 242.787 & +54.950 & 24 & 1.27 & 1.14 & 1.07 & 1.09 \\
MD09 & 334.188 & +00.283 & 9 & 1.26 & 1.15 & 1.02 & 1.02 \\
MD10 & 352.312 & -00.433 & 7 & 1.26 & 1.18 & 1.01 & 1.03 \\
& & & & & & &
\end{tabular}

cadence images in the grizy $y_{\mathrm{P} 1}$ bands of the MDF fields listed in Table 1. In this paper, we exclusively use SNe Ia detected in the MDFs.

The MDF exposure times in the five filters are listed in Table 2. Observations of three to five MDFs are taken each night and the filters are cycled through in the pattern: $g_{\mathrm{P} 1}$ and $r_{\mathrm{P} 1}$ in the same night (dark time), followed by $i_{\mathrm{P} 1}$ and $z_{\mathrm{P} 1}$ on the subsequent second and third night, respectively. Around full moon only $y_{\mathrm{P} 1}$ data are taken. Any one epoch consists of eight dithered exposures of $8 \times 113 \mathrm{~s}$ for $g_{\mathrm{P} 1}$ and $r_{\mathrm{P} 1}$ or $8 \times 240 \mathrm{~s}$ for the other three, giving nightly stacked images of 904 and $1920 \mathrm{~s}$ duration. For the current analysis, we do not use the $y_{\mathrm{P} 1}$ band. However, in the future, the $y_{\mathrm{P} 1}$ band data may prove useful for cosmological analysis of SNe Ia because measurements in this band are less affected by dust in the near infrared. Due to weather and the occasional technical downtime, the effective cadence varies significantly from season to season. On average, our cadence is 6 detections in 10 days, with a 5 day gap during bright time when the MDFs are exclusively observed in $y_{\mathrm{P} 1}$.

\section{TRANSIENT ALERT SYSTEM}

The depth of the MDF survey is ideal for detecting SNe Ia up to a redshift of $z \approx 0.7$, and the cadence provides well-sampled, multi-band light curves. In this section, we describe how the MDF data are reduced and the objects of interest are identified.

The PS1 image processing pipeline (IPP) system (Magnier 2006) performs flat fielding on each individual image, using white-light flat-field images of a dome screen, in combination with an illumination correction obtained by rastering sources across the field of view (FOV). After determining an initial astrometric solution (Magnier et al. 2008), the flat-fielded images were then warped onto the tangent plane of the sky, using a flux conserving algorithm.

For the MDFs, there are several (typically eight) dithered images per filter in a given night. This allows for the removal of defects like cosmic rays and satellite streaks before they are combined into a nightly stacked image using a varianceweighted scheme. The nightly MDF stacked images are processed through a frame-subtraction analysis using the photpipe pipeline that members of our team developed for the SuperMACHO and ESSENCE surveys (Rest et al. 2005; Garg et al. 2007; Miknaitis et al. 2007). This robust and well-tested system determines the appropriate spatially varying convolution kernel $^{21}$ needed to match an image with a template image, and then

\footnotetext{
21 http://www.astro.washington.edu/users/becker/hotpants.html
}

Table 2

MDF Cadence

\begin{tabular}{lccc}
\hline \hline Night & Filter & $\begin{array}{c}\text { Exposure Time } \\
(\mathrm{s})\end{array}$ & $\begin{array}{c}5 \sigma \text { Depth } \\
(\mathrm{AB} \mathrm{mag})\end{array}$ \\
\hline 1 & $g_{\mathrm{P} 1, r_{\mathrm{P} 1}}$ & $8 \times 113$ each & $23.1,23.3$ \\
2 & $i_{\mathrm{P} 1}$ & $8 \times 240$ & 23.2 \\
3 & $z_{\mathrm{P} 1}$ & $8 \times 240$ & 22.8 \\
Repeats & & $8 \times 240$ & 21.9 \\
FM \pm 3 & $y_{\mathrm{P} 1}$ & & \\
\hline
\end{tabular}

performs a subtraction of the two images. We then detect significant flux excursions in the difference images using a modified version of DoPHOT (Schechter et al. 1993).

The SNe Ia discussed in this work were discovered during the first $\sim 1.5 \mathrm{yr}$ of the survey. At the beginning of the survey, we had not yet obtained sufficient observations to construct deep template images for the $\mathrm{SNe}$ search. Instead, we typically obtained two epochs of observations in each filter with double the usual exposure time to use as templates. We subtracted these templates from later observations to search for transient objects. The use of two templates reduced false detections from imperfections in a single template. After this initial period, we generated deep reference images from a subset of images with good image quality and low sky background. These deep reference images were subsequently used as templates for the $\mathrm{SNe}$ search. The template images used for the search with photpipe are different from the deeper ones used for the final light curves created by transphot, as discussed in Section 5.1.

Once photometry is performed on the difference images, we apply a set of conditions before flagging an excursion in flux at a given position as a potential transient source. Typical conditions are as follows.

1. Positive detections with a signal-to-noise ratio $(\mathrm{S} / \mathrm{N}) \geqslant 4$ in at least three griz $_{\mathrm{P} 1}$ images within a time window of 10 days.

2. Detections in subtractions using at least two distinct templates.

3. No previous alert at that position.

The $\mathrm{S} / \mathrm{N}$ cut significantly reduces the number of false positives and artifacts. Similarly, requiring detections in subtractions versus two templates reduces spurious detections related to artifacts in a particular template. Our requirement of no previous alert at a particular position prevents variable sources from being continually flagged. The parameters related to the search were adjusted several times throughout the survey. There was a constant evolution of the parameters to optimize source detection as we gained experience with the data. However, there were also cases where we would modify the parameters at a given time for testing purposes or for immediate goals such as increasing the number of young detected objects just prior to a spectroscopic follow-up observing run.

An important aspect of our reduction scheme is to perform point-spread function (PSF) fitting photometry on all difference images at the location of any transient candidate, which we call "forced photometry." This is particularly helpful for discriminating between $\mathrm{SNe}$ and active galactic nuclei (AGNs), since many AGNs show low-level, long timescale variability in previous epochs that may not be above the detection limit in a single image, but clearly detectable over many epochs. It also can provide additional data when a $\mathrm{SN}$ is faint (particularly when it is rising) or if the image quality is poor (for instance when there are clouds). 

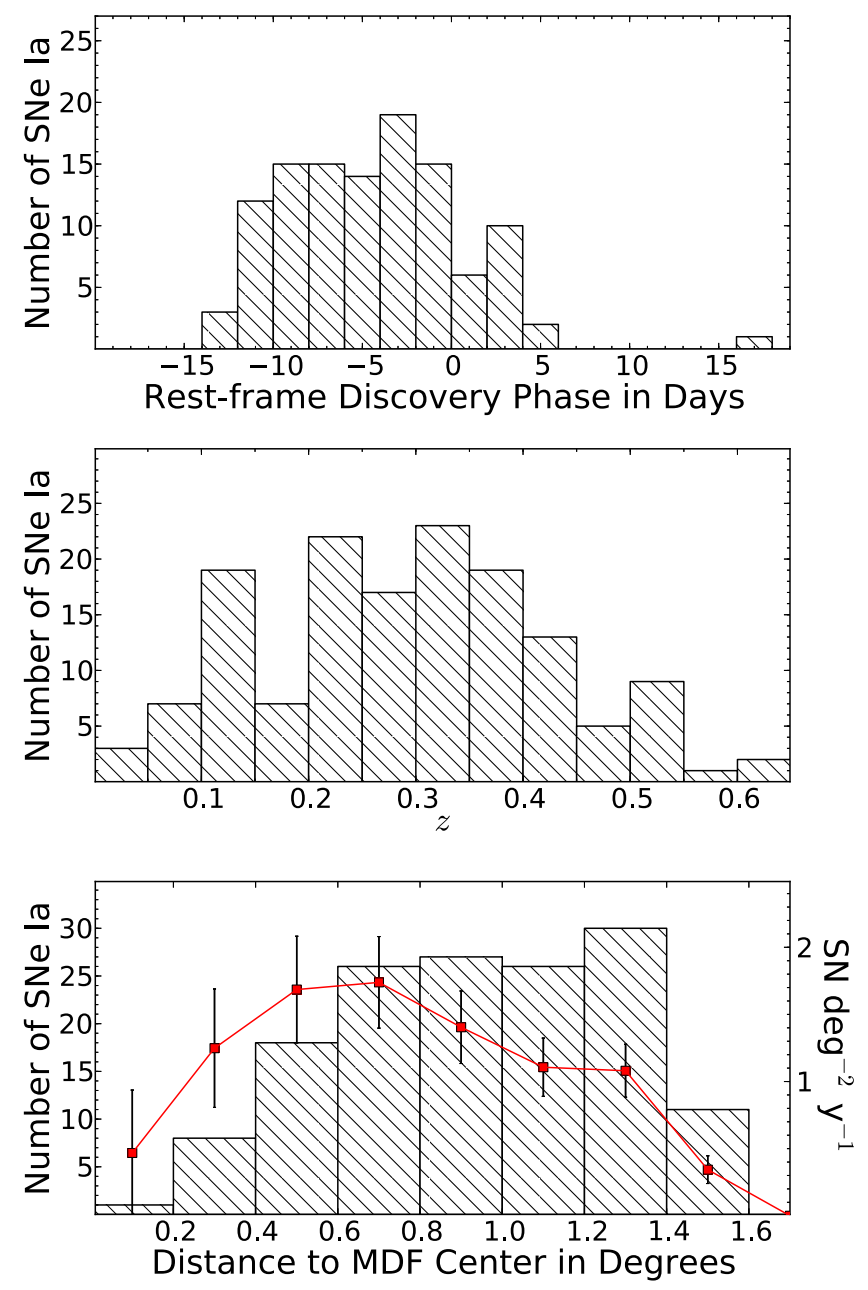

Figure 1. PS1 MDF survey characteristics for all spectroscopically confirmed PS1 SNe Ia. Upper panel: histogram of the number of SNe Ia with respect to the phase of discovery in the rest frame. Middle panel: histogram of the redshift distribution. Lower panel: histogram of SNe Ia as a function of distance to the MDF center. The observed SNe rate per constant area is shown with the red symbols and line. The rate is constant within the uncertainties to a distance of $\sim 1.3$ deg from center.

(A color version of this figure is available in the online journal.)

Each transient candidate that passes our stated conditions is sorted into one of five categories: transient, possible transient, variable, asteroid, and artifact. Transients are identified as sources with no variability in previous epochs, a "smooth" light curve, and either previous "in-season" non-detections or a clear offset from a host galaxy. Both because of possible confusion with AGNs and subtraction artifacts, transients that are visually offset from a galaxy nucleus are easier to identify than those that are coincident with a nucleus. In cases where the identification is ambiguous either due to low $\mathrm{S} / \mathrm{N}$ or possible variability in previous epochs, the object is classified as a possible transient.

We attempt to err on the side of inclusion to maximize the number of transients discovered. We refrain from classifying objects as "non-transient" until there is significant evidence that they are not transients (such as past variability, clear indication of the source being an artifact, or spectroscopy from an exterior catalog). We re-evaluate and possibly re-classify each candidate object as we acquire more points on its light curve.

As shown by the upper panel of Figure 1, we discovered almost all of our spectroscopically confirmed SNe Ia at phases between -12 and +4 days.
A parallel, independent search for transients is done at Queen's University Belfast based on difference images that are generated in Hawaii through the IPP system and this is described in McCrum et al. (2014). The correlation between the discoveries in photpipe and the IPP based "Transient Science Server" is excellent, with virtually all high significance transients detected in both systems. Some of the SNe in this sample were originally selected for spectroscopic classification from the IPP-based catalogs, but all were also detected through photpipe and all the photometry discussed in this paper is exclusively from the photpipe system.

\section{SPECTROSCOPIC PRIORITIZATION AND FOLLOW-UP SPECTROSCOPY}

Several spectroscopic programs rely on targets generated from the PS1/photpipe data stream. The primary source of classification spectra is a multipurpose CfA program at the MMT to observe PS1 transients (PI: Berger). Other spectra were obtained through Gemini programs to observe exotic transients and superluminous SNe (PIs: Berger, Smartt, and Chornock), a Gemini program to observe transients with faint hosts (PI: Tonry), a Magellan program to obtain high-S/N spectra of SNe Ia (PI: Foley), and Magellan and William Herschel programs to observe unusual transients and superluminous SN (PIs: Berger, Chornock, and Smartt). Ongoing programs that will create a larger sample of well-observed SNe Ia from PS1 include a program on Gemini (PI: Foley) and at Keck using NASA time (PI: Kirshner) to select targets for further study in the rest frame IR with the Hubble Space Telescope (HST). The target selection for each program varies significantly, but all begin with identifying potential targets from the PS1/photpipe data stream. We only have sufficient spectroscopic resources to obtain spectra of $\sim 10 \%$ of the PS1 transients, so potential targets must then pass several selection criteria, which again depend on the program. The most important criteria are position and brightness. Magellan and Gemini-South can only point to the five equatorial and southern MDFs. Observations at Magellan and the MMT are generally limited to targets with $r_{\mathrm{P} 1}<22 \mathrm{mag}$, the William Herschel Telescope (WHT) is limited to $r_{\mathrm{P} 1}<21.5 \mathrm{mag}$, while fainter targets can be observed at Gemini. Generally, an effort was made to observe transients close to their peak brightness. Several programs specifically target transients with very faint host galaxies, while others attempt to observe transients with host galaxies that have a particular photo- $z$. Although many transients are targeted specifically as potential SNe Ia, a significant number of SNe Ia in our sample were originally targeted as possible non-SNe Ia transients.

Spectroscopic observations of PS1 targets were obtained with a wide variety of instruments: the Blue Channel Spectrograph (Schmidt et al. 1989) and Hectospec (Fabricant et al. 2005) on the $6.5 \mathrm{~m}$ MMT, the Gemini Multi-Object Spectrographs (GMOS; Hook et al. 2004) on both Gemini North and South, the Low Dispersion Survey Spectrograph-3 $\left(\mathrm{LDSS}^{22}\right)$ and the Magellan Echellette (MagE; Marshall et al. 2008) on the $6.5 \mathrm{~m}$ Magellan Clay telescope, and the Inamori-Magellan Areal Camera and Spectrograph (IMACS; Dressler et al. 2011) on the $6.5 \mathrm{~m}$ Magellan Baade telescope, and the ISIS spectrograph on the WHT. Nod-and-shuffle techniques (Glazebrook \& BlandHawthorn 2001) were used with some GMOS (North and South) observations to improve sky subtraction in the red portion of the spectrum.

\footnotetext{
22 http://www.lco.cl/telescopes-information/magellan/instruments-1/ldss-3-1
} 


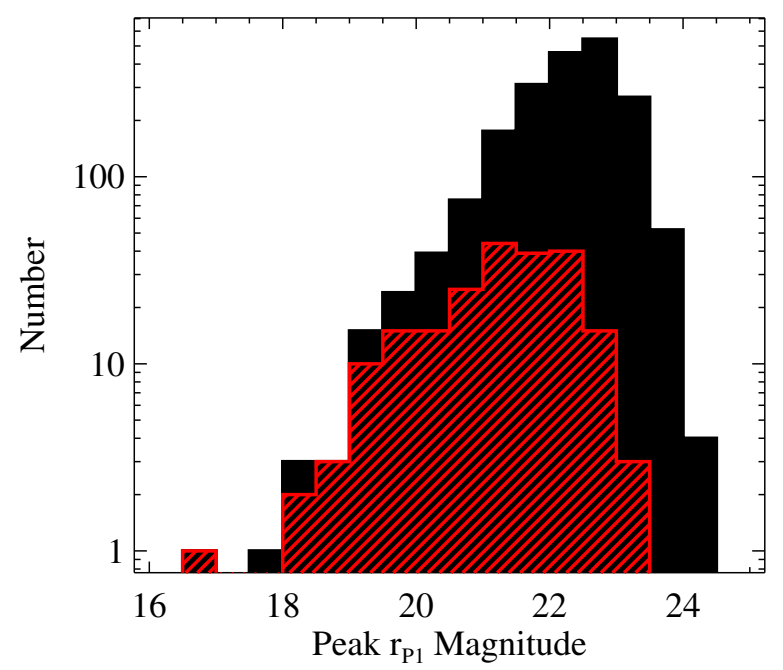

Figure 2. Histogram of $r_{\mathrm{P} 1}$ peak magnitudes of spectroscopically confirmed SNe Ia (red) and events classified as highly likely SNe Ia (black) based on light curve fitting with PSNID (Sako et al. 2011).

(A color version of this figure is available in the online journal.)

Standard CCD processing and spectrum extraction were accomplished with IRAF. ${ }^{23}$ The data were extracted using the optimal algorithm of Horne (1986). Low-order polynomial fits to calibration-lamp spectra were used to establish the wavelength scale. Small adjustments derived from night sky lines in the object frames were applied. For the MagE spectra, the sky was subtracted from the images using the method described by Kelson (2003). We employed our own IDL routines to flux calibrate the data and remove telluric lines using the wellexposed continua of the spectrophotometric standards (Wade \& Horne 1988; Foley et al. 2003, 2009a; Silverman et al. 2012a).

With fully calibrated spectra, we classify the PS1 objects by physical origin. SNe, having broad $\left(\sim 10,000 \mathrm{~km} \mathrm{~s}^{-1}\right)$ spectral features, are very distinct from AGNs, galaxies, stars, and other astrophysical objects. However, it can occasionally be difficult to distinguish among SN types. High-redshift SN spectra typically have low $\mathrm{S} / \mathrm{N}$ and considerable host-galaxy contamination, while spectra of the highest redshift SNe will lack the Si II $\lambda 6355$ feature. We have implemented the SNID algorithm (Blondin \& Tonry 2007) to aid in SN classification.

For SNID, as well as any SN spectral fitting routine, the output is dependent on the input parameters such as wavelength range. Because of the different approaches and the various input parameters, it is possible for different fitters to suggest different classifications. However, humans ultimately classify each object, and reasonable inputs to the fitters should yield similar results. Unless there is a confident classification, the SN is discarded.

In Table 3, we present a full list of our observations (date of observation, telescope/instrument, and exposure times) of all spectroscopically confirmed SNe Ia detected in the PS1 photometric data spanning from 2009 September to the end of 2011 May. We also include information about the nature of each object (redshift, phase, and light curve shape) in Table 4. The middle panel of Figure 1 shows the redshift distribution of the spec-

23 IRAF: the Image Reduction and Analysis Facility is distributed by the National Optical Astronomy Observatory, which is operated by the

Association of Universities for Research in Astronomy (AURA), Inc., under cooperative agreement with the National Science Foundation (NSF). troscopically confirmed SNe Ia. Since this period includes the ramp-up of PS1 operations, our detection efficiency increased over time due to improved reductions, deeper templates, and longer history to identify and differentiate between the transients and variables. In addition, access to telescopes, weather, and PS1 downtime influenced our spectroscopic follow-up efficiency. In particular, the annual monsoon rainstorms at the MMT in July along with the August telescope shutdown resulted in poor follow up for the summer fields (primarily MDFs 9 and 10). Our spectroscopic follow-up rate for SNe Ia is $\sim 1.5 \mathrm{deg}^{-2} \mathrm{yr}^{-1}$ and constant within the uncertainties out to a radius of $\sim 1.3 \mathrm{deg}$ in each field (see lower panel of Figure 1). In Figure 2, we show the peak magnitudes of spectroscopically confirmed SNe Ia as well as events having a high likelihood of being SNe Ia based on light curve only classification (Sako et al. 2011). Unsurprisingly, spectroscopic follow-up favored brighter transients. As Figure 2 indicates, our $5 \sigma$ detection limit for identifying transients provides good light curves for SNe Ia with $m<24$, while our spectroscopic sample consists principally of objects with $m<22$. The Malmquist bias introduced by this selection is discussed in Section 8.

\section{LIGHT CURVES WITH THE TRANSIENT PHOTOMETRY PIPELINE}

The goal of the transient alert system is to produce light curves of potential transients in a quick and robust way to distribute them for validation and follow-up spectroscopy. However, for measuring distance, the main focus is on minimizing the random and systematic uncertainties in the light curves. Therefore, we have set up a transient photometry pipeline (transphot) that is closely related to photpipe and uses most of its features, but differs in some significant ways, described below. The overall process can be summarized as follows. Templates from stacking multiple images at a given sky position are constructed to allow for accurate world coordinate system (WCS) registration and image subtraction to measure photometry. From the subtracted images of SNe, a SN centroid is determined and forced photometry is performed at that position on all images, using the PSF determined from neighboring stars for that epoch. Afterward, the errors of the SN measurements and the baseline flux is adjusted so that the reduced chi-squared of the measurements of the SNe that do not have SN light will be unity.

\subsection{Templates}

The templates are created through a custom calibration and stacking process. The eight dithers from a single night are reduced and combined into a "nightly stack" by IPP with a variance-weighted scheme (see Section 3).

The "deep stack" is constructed by combining nightly stacks (typically $\sim 30$ ), weighted by the product of the inverse variance and the inverse area of the PSF. This prescription is nearly optimal for point-source detection and photometry (Tonry et al. 2012a). The typical seeing values determined by DoPHOT for deep stacks that use all available epochs are shown in Table 1 (Tonry et al. 2012a). The typical $5 \sigma$ detection limit in these deep stacks is $\sim 25.2,25.4,25.4,25.0$ for griz $_{\mathrm{P} 1}$, respectively. To create the deep templates for a given $\mathrm{SN}$, we only use epochs with no SN flux in the images. This ensures the spatial consistency of the PSF, and also minimizes errors introduced by imperfect image subtraction kernels. In general, if there is no SN flux in the template, only the extended flux contribution from the galaxy is subtracted from the image. Such spatially extended 
Table 3

Pan-STARRS1 Spectroscopic Sample

\begin{tabular}{|c|c|c|c|}
\hline PS1 Name & Spectra MJD's & Telescopes & $z_{\text {hel }}$ \\
\hline PS1-0909006 & 55098 & Gem-N & $0.284(0.001)$ \\
\hline PS1-0909010 & 55105 & Gem-N & $0.270(0.010)$ \\
\hline PS1-0910012 & $55116,55118,55121,55142$ & Gem-S, NOT, Magellan, Gem-S & $0.079(0.001)$ \\
\hline PS1-0910016 & 55125 & Gem-N & $0.230(0.010)$ \\
\hline PS1-0910017 & 55125 & Gem-N & $0.320(0.010)$ \\
\hline PS1-0910018 & 55125 & Gem-N & $0.265(0.001)$ \\
\hline PS1-0910020 & 55124 & Gem-S & $0.242(0.001)$ \\
\hline PS1-0910021 & 55125 & Gem-S & $0.256(0.001)$ \\
\hline PS1-10c & 55213 & Gem-N & $0.152(0.001)$ \\
\hline PS1-10d & 55216 & Magellan & $0.231(0.001)$ \\
\hline PS1-10e & 55217 & Magellan & $0.245(0.001)$ \\
\hline PS1-10f & 55214 & Gem-S & $0.380(0.010)$ \\
\hline PS $1-10 \mathrm{~g}$ & 55217 & Magellan & $0.137(0.001)$ \\
\hline PS1-10h & 55215,55527 & Magellan, MMT & $0.250(0.010)$ \\
\hline PS1-10i & 55217 & Magellan & $0.150(0.001)$ \\
\hline PS1-10j & $55216,55249,55293$ & Gem-N, MMT, MMT & $0.436(0.001)$ \\
\hline PS1-10k & 55216 & Magellan & $0.340(0.001)$ \\
\hline PS1-101 & 55216 & Magellan & $0.370(0.001)$ \\
\hline PS1-10m & 55216,55297 & Gem-S, MMT & $0.618(0.001)$ \\
\hline PS1-10n & 55217 & Magellan & $0.460(0.001)$ \\
\hline PS1-10o & 55216 & Magellan & $0.220(0.001)$ \\
\hline PS1-10p & 55217 & Magellan & $0.310(0.001)$ \\
\hline PS1-10r & 55251 & MMT & $0.118(0.001)$ \\
\hline PS1-10s & 55221 & Gem-S & $0.101(0.001)$ \\
\hline PS1-10u & 55217 & NOT & $0.183(0.001)$ \\
\hline PS1-10v & 55247 & WHT & $0.098(0.001)$ \\
\hline PS1-10w & 55244 & FLWO & $0.031(0.001)$ \\
\hline PS1-10z & 55250 & WHT & $0.140(0.001)$ \\
\hline PS1-10af & 55233,55268 & NOT, Gem-N & $0.244(0.001)$ \\
\hline PS1-10ag & 55263 & NOT & $0.081(0.001)$ \\
\hline PS1-10aj & 55233 & Gem-N & $0.195(0.001)$ \\
\hline PS1-10ht & 55290 & MMT & $0.260(0.010)$ \\
\hline PS1-10hu & 55288 & WHT & $0.126(0.001)$ \\
\hline PS1-10ia & 55295 & Keck & $0.409(0.001)$ \\
\hline PS1-10if & 55288,55297 & WHT, MMT & $0.150(0.001)$ \\
\hline PS1-10ig & 55290 & MMT & $0.260(0.001)$ \\
\hline PS1-10ik & 55293 & MMT & $0.230(0.001)$ \\
\hline PS1-10im & 55293 & MMT & $0.510(0.001)$ \\
\hline PS1-10io & 55319 & WHT & $0.220(0.001)$ \\
\hline PS1-10ir & 55355 & WHT & $0.250(0.001)$ \\
\hline PS1-10iv & 55330 & Gem-N & $0.369(0.001)$ \\
\hline PS1-10iw & 55331 & Gem-N & $0.447(0.001)$ \\
\hline PS1-10ix & 55330 & Gem-N & $0.381(0.001)$ \\
\hline PS1-10iy & 55331 & Gem-N & $0.443(0.001)$ \\
\hline PS1-10ji & 55363 & MMT & $0.231(0.001)$ \\
\hline PS1-10jk & 55363 & MMT & $0.430(0.001)$ \\
\hline PS1-10j1 & 55363 & MMT & $0.530(0.001)$ \\
\hline PS1-10jo & 55364 & MMT & $0.241(0.001)$ \\
\hline PS1-10jp & 55364 & MMT & $0.387(0.001)$ \\
\hline PS1-10jq & 55364 & MMT & $0.459(0.001)$ \\
\hline PS1-10jt & 55364 & MMT & $0.336(0.001)$ \\
\hline PS1-10ju & 55364 & MMT & $0.323(0.001)$ \\
\hline PS1-10jv & 55364 & MMT & $0.360(0.001)$ \\
\hline PS1-10jw & 55364 & MMT & $0.360(0.001)$ \\
\hline PS1-10jz & 55365 & MMT & $0.550(0.001)$ \\
\hline PS1-10kc & 55365 & MMT & $0.347(0.001)$ \\
\hline PS1-10kd & 55365 & MMT & $0.331(0.001)$ \\
\hline PS1-10kf & 55365 & MMT & $0.450(0.001)$ \\
\hline PS1-10kg & 55365 & MMT & $0.420(0.010)$ \\
\hline PS1-10ki & 55365 & MMT & $0.379(0.001)$ \\
\hline PS1-10kj & 55365 & MMT & $0.350(0.010)$ \\
\hline PS1-10kl & 55365 & MMT & $0.443(0.001)$ \\
\hline PS1-10kv & 55384 & Gem-N & $0.530(0.001)$ \\
\hline PS1-10nq & 55351,55355 & UH88, WHT & $0.035(0.001)$ \\
\hline PS1-10nu & 55422 & Magellan & $0.065(0.001)$ \\
\hline PS1-10acx & 55288 & MMT & $0.350(0.001)$ \\
\hline
\end{tabular}


Table 3

(Continued)

\begin{tabular}{|c|c|c|c|}
\hline PS1 Name & Spectra MJD's & Telescopes & $z_{\text {hel }}$ \\
\hline PS1-10aeq & 55475 & $\mathrm{APO}$ & $0.066(0.001)$ \\
\hline PS1-10afu & 55473 & MMT & $0.338(0.001)$ \\
\hline PS1-10agw & 55471 & MMT & $0.330(0.010)$ \\
\hline PS1-10ahk & 55479 & MMT & $0.431(0.001)$ \\
\hline PS1-10ahl & 55471 & MMT & $0.635(0.001)$ \\
\hline PS1-10axm & 55485 & Gem-N & $0.510(0.010)$ \\
\hline PS1-10axx & 55487 & MMT & $0.027(0.001)$ \\
\hline PS1-10ayb & 55514 & MMT & $0.140(0.001)$ \\
\hline PS1-10bhe & 55487 & MMT & $0.145(0.001)$ \\
\hline PS1-10bji & 55514 & MMT & $0.148(0.001)$ \\
\hline PS1-10bjn & 55528 & MMT & $0.290(0.010)$ \\
\hline PS1-10bjz & 55540 & MMT & $0.310(0.010)$ \\
\hline PS1-10bka & 55507 & Gem-N & $0.247(0.001)$ \\
\hline PS1-10bkf & 55539 & MMT & $0.172(0.001)$ \\
\hline PS1-10bki & 55539 & MMT & $0.430(0.001)$ \\
\hline PS1-10blh & 55539 & MMT & $0.319(0.001)$ \\
\hline PS1-10blp & 55515,55540 & MMT, MMT & $0.217(0.001)$ \\
\hline PS1-10bls & 55540 & MMT & $0.276(0.001)$ \\
\hline PS1-10bmb & 55539 & MMT & $0.360(0.010)$ \\
\hline PS1-10bmk & 55539 & MMT & $0.103(0.001)$ \\
\hline PS1-10byj & 55547 & Gem-N & $0.511(0.001)$ \\
\hline PS1-10byn & 55540 & MMT & $0.179(0.001)$ \\
\hline PS1-10byo & 55558 & MMT & $0.290(0.001)$ \\
\hline PS1-10byq & 55539 & MMT & $0.210(0.010)$ \\
\hline PS1-10byr & 55540 & MMT & $0.240(0.010)$ \\
\hline PS1-10bys & 55540 & MMT & $0.203(0.001)$ \\
\hline PS1-10bzo & 55558 & MMT & $0.380(0.001)$ \\
\hline PS1-10bzp & 55559 & MMT & $0.540(0.001)$ \\
\hline PS1-10bzt & 55558 & MMT & $0.420(0.001)$ \\
\hline PS1-10bzu & 55558 & MMT & $0.350(0.001)$ \\
\hline PS1-10bzy & 55558 & MMT & $0.200(0.001)$ \\
\hline PS1-10cad & 55558 & MMT & $0.270(0.010)$ \\
\hline PS1-10cay & 55558 & MMT & $0.250(0.010)$ \\
\hline PS1-10cbb & 55559 & MMT & $0.220(0.010)$ \\
\hline PS1-10cbu & 55570 & MMT & $0.400(0.001)$ \\
\hline PS1-11e & 55570 & MMT & $0.320(0.001)$ \\
\hline PS1-11p & 55573 & Magellan & $0.480(0.001)$ \\
\hline PS1-11s & 55573 & Magellan & $0.400(0.001)$ \\
\hline PS1-11t & 55573 & Magellan & $0.450(0.001)$ \\
\hline PS1-11w & 55571 & MMT & $0.174(0.001)$ \\
\hline PS1-11aj & 55571 & MMT & $0.106(0.001)$ \\
\hline PS1-11ao & 55570 & MMT & $0.336(0.001)$ \\
\hline PS1-11at & 55570 & MMT & $0.320(0.001)$ \\
\hline PS1-11bg & 55590 & Magellan & $0.329(0.001)$ \\
\hline PS1-11bh & 55573 & Magellan & $0.350(0.001)$ \\
\hline PS1-11bk & 55592 & Magellan & $0.160(0.001)$ \\
\hline PS1-11br & 55586 & Gem-S & $0.300(0.010)$ \\
\hline PS $1-11 \mathrm{cn}$ & 55592 & Gem-N & $0.250(0.010)$ \\
\hline PS1-11co & 55591 & Gem-S & $0.230(0.010)$ \\
\hline PS1-11fi & 55614 & MMT & $0.082(0.001)$ \\
\hline PS1-11gh & 55626 & Magellan & $0.220(0.010)$ \\
\hline PS1-11gr & 55617 & APO & $0.105(0.001)$ \\
\hline PS1-11iv & 55615 & MMT & $0.293(0.001)$ \\
\hline PS1-11jo & 55615 & MMT & $0.330(0.001)$ \\
\hline PS1-11kk & 55615 & MMT & $0.300(0.010)$ \\
\hline PS1-11mq & 55656,55687 & MMT, MMT & $0.210(0.001)$ \\
\hline PS1-11mz & 55651 & $\mathrm{APO}$ & $0.101(0.001)$ \\
\hline PS1-11sk & 55687 & MMT & $0.270(0.001)$ \\
\hline PS1-11um & 55687 & MMT & $0.064(0.001)$ \\
\hline PS1-11uo & 55687 & MMT & $0.310(0.001)$ \\
\hline PS1-11uw & 55687 & MMT & $0.300(0.001)$ \\
\hline PS1-11vb & 55687 & MMT & $0.230(0.010)$ \\
\hline PS1-11wv & 55684 & APO & $0.132(0.001)$ \\
\hline PS1-11xc & 55716 & MMT & $0.328(0.001)$ \\
\hline PS1-11xw & 55723 & MMT & $0.270(0.001)$ \\
\hline PS1-11yj & 55717 & MMT & $0.107(0.001)$ \\
\hline
\end{tabular}


Table 3

(Continued)

\begin{tabular}{lccc}
\hline \hline PS1 Name & Spectra MJD's & Telescopes & $z_{\text {hel }}$ \\
\hline PS1-11yr & 55723 & Gem-N & $0.530(0.001)$ \\
PS1-11yu & 55716 & MMT & $0.360(0.010)$ \\
PS1-11zd & 55716 & MMT & $0.100(0.001)$ \\
PS1-11zg & 55721 & MMT & $0.370(0.001)$ \\
PS1-11zu & 55717 & MMT & $0.360(0.001)$ \\
PS1-11zv & 55717 & MMT & $0.350(0.001)$ \\
PS1-11zw & 55717 & MMT & $0.423(0.001)$ \\
PS1-11aaw & 55717 & MMT & $0.510(0.010)$ \\
PS1-11abm & 55745 & Gem-N & $0.320(0.001)$ \\
PS1-11aea & 55744 & Gem-N & $0.300(0.001)$ \\
PS1-11aij & Gem-N & $0.510(0.001)$ \\
PS1-11ajs & 55769 & MMT & $0.230(0.010)$ \\
PS1-11ala & 55808 & MMT & $0.370(0.010)$ \\
PS1-11alv & 55808 & MMT & $0.145(0.001)$ \\
PS1-11ams & 55807 & Magellan & $0.140(0.001)$ \\
\hline
\end{tabular}

Notes. Spectroscopic information for all spectroscopically classified Pan-STARRS1 SN Ia from 2009 September to 2011 May.

sources are less susceptible to imperfections in the kernel than point sources, minimizing the difference image residuals at the position of the SN. The main disadvantage of templates that only use epochs that contain no SN flux is the template's lower $\mathrm{S} / \mathrm{N}$ than templates that use all epochs. However, since even in these templates the $\mathrm{S} / \mathrm{N}$ is still significantly higher than the $\mathrm{S} / \mathrm{N}$ in the single epochs (nightly stacks), the $\mathrm{S} / \mathrm{N}$ in the difference image is only marginally degraded.

\subsection{SN Centroids}

Determining the correct position of the $\mathrm{SNe}$ is important for two reasons. (1) For many epochs, the flux of the $\mathrm{SNe}$ is below the detection limit. (2) Even if the flux of the $\mathrm{SNe}$ is above the detection limit, its measurement is biased toward higher fluxes due to Poisson fluctuations in the sky background (see Section 5.3.2 for a detailed discussion).

In order to achieve good centroiding, an accurate WCS solution for each image is needed. Additionally, the uncertainties in the position vary significantly due to their strong dependence on the $\mathrm{S} / \mathrm{N}$ of the flux and the seeing of the image. Therefore it is important to have an accurate understanding of the uncertainties in order to calculate an unbiased weighted average centroid. In Appendix A, we determine the accuracy of our WCS solution. The astrometric uncertainty $\sigma_{a}$ of a given detection has a floor mostly due to pixelization $\left(\sigma_{a 1}\right)$, and in addition a random error $\sigma_{a 2}$, which is scaled by the square of the ratio of the FWHM of the image and the $\mathrm{S} / \mathrm{N}$ of the detection:

$$
\sigma_{a}^{2}=\sigma_{a 1}^{2}+\sigma_{a 2}^{2}\left(\frac{\mathrm{FWHM}}{\mathrm{S} / \mathrm{N}}\right)^{2}
$$

As described in Appendix A, for PS1 we conservatively use $\sigma_{a 1}=40$ mas and $\sigma_{a 2}=1.5$ to calculate the astrometric uncertainty of a single detection. The detections are grouped into transient objects, for which the $3 \sigma$ clipped and weighted average centroids are calculated. The accuracy of the centroid has a small effect on the accuracy of the photometry (see Figure 5), and we discuss how we correct for this effect as described in Section 5.3.2 and Appendix D.

\subsection{Light Curves}

The modeling and fitting of the PSF is one of the main potential sources of systematic biases in the photometry. For the alert system, we use a customized version of DoPHOT, which is quick, robust, and produces adequate photometry for alerts. However, this DoPHOT version uses an analytic PSF that does not capture the non-Gaussian PSF tails in the PS1 images, which was especially important during the first year while the optical system was being perfected. This can introduce biases at the level of a few percent between faint and bright stars. For more precise photometry, we employ DAOPHOT (Stetson 1987) as implemented in IDL, which fits an empirical correction in addition to the Gaussian model.

\subsubsection{Linearity}

Figure 3 shows the magnitude difference between the deep and nightly stacks, $\Delta m=m_{\text {deep }}-m_{\text {nightly, versus the deep }}$ stack magnitudes (black dots) for the various PS1 filters. For magnitudes fainter than 17.5, the agreement is excellent, and the average residual is on the order of $1 \mathrm{mmag}$ and in general within the uncertainties (with the exception of the faintest magnitudes, where the expected Malmquist bias can be seen). A small systematic bias can be seen for the very brightest magnitude bins. In particular, for the $i_{\mathrm{P} 1}$ filter, the average $\Delta m=0.017 \pm 0.0013 \mathrm{mag}$ for the bin with $i_{\mathrm{p} 1 \text {,deep }} \approx 17 \mathrm{mag}$ is significantly above zero. This is most likely the result of imperfect stacking algorithms of stars close to or at the saturation limit, and we therefore exclude all stars brighter than $m=17.5 \mathrm{mag}$ for determining the zero point of an image or the difference image kernel. No detections from our SNe light curves fall into this magnitude range, therefore we set the systematic bias due to non-linearity of the photometry to 1 mmag (see Table 5).

We also tested the linearity of the PS1 detectors by comparing it to an outside catalog. We convert $r_{\text {SDSS }}$ from stripe 82 into PS1 natural system magnitudes, and calculate the difference, shown in Figure 4. For $r_{\text {SDSS }}<19$, the average differences are smaller than $1 \mathrm{mmag}$, which is an upper constraint on the linearity over this magnitude range. For larger magnitudes, we are susceptible to a Malmquist bias in the SDSS magnitudes. 
Table 4

Properties of the Pan-STARRS1 Spectroscopic Sample

\begin{tabular}{|c|c|c|c|c|c|}
\hline PS1 Name & R.A. (J2000) & Decl. (J2000) & MDF & $t_{\mathrm{disc}}$ & Cut \\
\hline PS1-0909006 & $22: 15: 48.000$ & 01:11:05.39 & 09 & 55083 & . \\
\hline PS1-0909010 & $02: 28: 28.368$ & $-04: 04: 44.08$ & 01 & 55089 & $\left|x_{1}\right|>3.0$ \\
\hline PS1-0910012 & $03: 29: 53.232$ & $-28: 05: 12.12$ & 02 & 55096 & Iax \\
\hline PS1-0910016 & $02: 21: 13.752$ & $-03: 54: 35.82$ & 01 & 55104 & $\left|x_{1}\right|>3.0$ \\
\hline PS1-0910017 & 02:21:06.600 & $-05: 01: 23.84$ & 01 & 55104 & $\cdots$ \\
\hline PS1-0910018 & $02: 22: 40.080$ & $-04: 01: 38.32$ & 01 & 55104 & $\cdots$ \\
\hline PS1-0910020 & $03: 38: 23.400$ & $-28: 15: 11.88$ & 02 & 55104 & LC incomplete \\
\hline PS1-0910021 & $03: 34: 28.800$ & $-27: 54: 30.24$ & 02 & 55104 & LC incomplete \\
\hline PS1-10c & 08:37:18.937 & $44: 20: 01.44$ & 03 & 55207 & $\cdots$ \\
\hline PS1-10d & $03: 33: 27.948$ & $-28: 22: 17.48$ & 02 & 55207 & $\cdots$ \\
\hline PS1-10e & 09:58:59.758 & 03:09:27.11 & 04 & 55208 & $\cdots$ \\
\hline PS1-10f & 09:59:54.221 & $02: 24: 42.84$ & 04 & 55208 & Close to center \\
\hline PS1-10g & $02: 23: 30.711$ & $-04: 38: 10.76$ & 01 & 55206 & $\cdots$ \\
\hline PS1-10h & $02: 24: 15.945$ & $-03: 11: 11.53$ & 01 & 55206 & $\cdots$ \\
\hline PS1-10i & $02: 20: 22.959$ & $-04: 40: 21.03$ & 01 & 55206 & $\cdots$ \\
\hline PS1-10j & $10: 49: 14.220$ & 58:28:02.04 & 05 & 55212 & $\cdots$ \\
\hline PS1-10k & 10:00:27.616 & $02: 41: 38.52$ & 04 & 55212 & $\cdots$ \\
\hline PS1-101 & 10:04:55.536 & $02: 35: 27.31$ & 04 & 55212 & $\cdots$ \\
\hline PS1-10m & 10:02:09.393 & 01:53:57.01 & 04 & 55212 & $\cdots$ \\
\hline PS1-10n & 10:03:14.385 & 03:08:39.21 & 04 & 55212 & $\cdots$ \\
\hline PS1-10o & 10:02:08.822 & $02: 43: 06.83$ & 04 & 55214 & $\cdots$ \\
\hline PS1-10p & 09:59:16.802 & $01: 36: 48.55$ & 04 & 55216 & $\cdots$ \\
\hline PS1-10r & $10: 44: 38.225$ & $57: 48: 40.04$ & 05 & 55212 & $\cdots$ \\
\hline PS1-10s & 09:55:57.231 & $02: 15: 48.47$ & 04 & 55212 & LC incomplete \\
\hline PS1-10u & $02: 19: 46.554$ & $-03: 12: 10.47$ & 01 & 55215 & Fit not converged \\
\hline PS1-10v & 10:03:26.078 & 01:01:45.91 & 04 & 55240 & $\cdots$ \\
\hline PS1-10w & $10: 42: 41.887$ & 58:50:37.77 & 05 & 55240 & $\cdots$ \\
\hline PS1-10z & $08: 36: 12.605$ & $44: 00: 25.52$ & 03 & 55240 & $\cdots$ \\
\hline PS1-10af & 10:48:07.061 & $56: 51: 00.54$ & 05 & 55212 & $\cdots$ \\
\hline PS1-10ag & $10: 57: 41.331$ & $57: 36: 48.36$ & 05 & 55251 & LC incomplete \\
\hline PS1-10aj & 08:46:19.241 & $44: 04: 22.97$ & 03 & 55214 & $\cdots$ \\
\hline PS1-10ht & 08:46:50.687 & $45: 29: 31.33$ & 03 & 55275 & LC incomplete \\
\hline PS1-10hu & $12: 25: 20.676$ & $46: 00: 54.75$ & 06 & 55275 & $\cdots$ \\
\hline PS1-10ia & $14: 11: 31.058$ & $53: 34: 46.48$ & 07 & 55275 & $\cdots$ \\
\hline PS1-10if & 10:00:21.196 & $01: 25: 05.25$ & 04 & 55275 & $\cdots$ \\
\hline PS1-10ig & $14: 07: 26.975$ & $53: 20: 34.49$ & 07 & 55275 & $\cdots$ \\
\hline PS1-10ik & $10: 45: 15.388$ & $58: 11: 33.76$ & 05 & 55275 & $\cdots$ \\
\hline PS1-10im & 10:50:05.881 & $58: 46: 38.71$ & 05 & 55288 & $\cdots$ \\
\hline PS1-10io & $10: 00: 44.015$ & $03: 24: 14.31$ & 04 & 55298 & $\cdots$ \\
\hline PS1-10ir & $10: 45: 09.371$ & $58: 33: 16.64$ & 05 & 55326 & $\cdots$ \\
\hline PS1-10iv & 10:02:00.450 & 02:03:37.82 & 04 & 55320 & LC incomplete \\
\hline PS1-10iw & $14: 17: 50.504$ & $52: 48: 03.77$ & 07 & 55326 & $\cdots$ \\
\hline PS1-10ix & $10: 48: 23.471$ & 57:08:53.54 & 05 & 55326 & $\cdots$ \\
\hline PS1-10iy & $14: 16: 28.707$ & $54: 03: 12.82$ & 07 & 55326 & $\cdots$ \\
\hline PS1-10ji & $16: 14: 28.674$ & $54: 43: 32.92$ & 08 & 55347 & $\cdots$ \\
\hline PS1-10jk & $16: 08: 52.639$ & 55:00:17.32 & 08 & 55348 & $\cdots$ \\
\hline PS1-10j1 & 14:07:14.923 & $53: 59: 51.40$ & 07 & 55348 & $\cdots$ \\
\hline PS1-10jo & $12: 16: 41.554$ & $46: 41: 24.09$ & 06 & 55353 & LC incomplete \\
\hline PS1-10jp & $14: 17: 18.567$ & $53: 58: 41.96$ & 07 & 55353 & $\cdots$ \\
\hline PS1-10jq & $12: 21: 46.461$ & $46: 39: 13.16$ & 06 & 55363 & LC incomplete \\
\hline PS1-10jt & 16:08:18.409 & $54: 54: 29.34$ & 08 & 55347 & $\cdots$ \\
\hline PS1-10ju & $16: 18: 15.872$ & $55: 23: 04.95$ & 08 & 55353 & $\cdots$ \\
\hline PS1-10jv & $16: 17: 47.706$ & 55:18:08.24 & 08 & 55353 & $\cdots$ \\
\hline PS1-10jw & $16: 13: 48.215$ & $55: 36: 30.73$ & 08 & 55353 & $\cdots$ \\
\hline PS1-10jz & $16: 06: 48.805$ & $54: 58: 51.36$ & 08 & 55353 & $\cdots$ \\
\hline PS1-10kc & $16: 18: 53.807$ & $55: 46: 58.47$ & 08 & 55353 & $\cdots$ \\
\hline PS1-10kd & $16: 05: 44.832$ & $56: 04: 27.90$ & 08 & 55353 & $\cdots$ \\
\hline PS1-10kf & $14: 11: 57.770$ & $52: 04: 18.65$ & 07 & 55362 & $\cdots$ \\
\hline PS1-10kg & $16: 01: 15.410$ & $54: 17: 53.54$ & 08 & 55362 & LC incomplete \\
\hline PS1-10ki & $12: 21: 59.469$ & $45: 50: 49.80$ & 06 & 55362 & LC incomplete \\
\hline PS1-10kj & $12: 14: 06.504$ & $46: 59: 32.29$ & 06 & 55362 & LC incomplete \\
\hline PS1-10k1 & $14: 14: 03.340$ & $53: 21: 43.87$ & 07 & 55362 & $\cdots$ \\
\hline PS1-10kv & $14: 10: 39.353$ & $53: 59: 22.76$ & 07 & 55353 & $\cdots$ \\
\hline PS1-10nq & $12: 11: 33.322$ & $47: 16: 29.36$ & 06 & 55344 & $\cdots$ \\
\hline PS1-10nu & $02: 27: 12.037$ & $-04: 32: 04.87$ & 01 & 55414 & $\cdots$ \\
\hline PS1-10acx & $16: 05: 54.089$ & $55: 11: 52.30$ & 08 & 55424 & $\cdots$ \\
\hline
\end{tabular}

Table 4

(Continued)

\begin{tabular}{|c|c|c|c|c|c|}
\hline PS1 Name & R.A. (J2000) & Decl. (J2000) & MDF & $t_{\text {disc }}$ & Cut \\
\hline S1-10aeq & $03: 29: 42.071$ & $-27: 52: 38.11$ & 02 & 55442 & $\ldots$ \\
\hline PS1-10afu & $16: 10: 40.664$ & $55: 55: 42.48$ & 08 & 55446 & LC incomplete \\
\hline PS1-10agw & $22: 18: 29.334$ & $00: 56: 29.35$ & 09 & 55449 & $\cdots$ \\
\hline PS1-10ahk & 02:19:56.728 & $-03: 57: 38.18$ & 01 & 55468 & $\cdots$ \\
\hline PS1-10ahl & $22: 15: 29.670$ & $00: 32: 30.87$ & 09 & 55468 & $\ldots$ \\
\hline PS1-10axm & $23: 33: 18.787$ & $-00: 57: 02.56$ & 10 & 55479 & $\cdots$ \\
\hline PS1-10axx & $08: 44: 15.659$ & $43: 18: 28.86$ & 03 & 55475 & $P<0.001$ \\
\hline PS1-10ayb & $03: 36: 17.301$ & $-28: 03: 05.44$ & 02 & 55482 & $\cdots$ \\
\hline PS1-10bhe & 08:40:03.838 & $43: 25: 17.23$ & 03 & 55475 & LC incomplete \\
\hline PS1-10bji & $03: 28: 29.494$ & $-26: 49: 42.58$ & 02 & 55500 & $\cdots$ \\
\hline PS1-10bjn & $23: 27: 37.793$ & $-01: 45: 44.83$ & 10 & 55493 & $\cdots$ \\
\hline PS1-10bjz & $23: 33: 10.781$ & $-01: 14: 07.02$ & 10 & 55501 & $\ldots$ \\
\hline PS1-10bka & $08: 46: 43.279$ & 44:00:12.56 & 03 & 55501 & LC incomplete \\
\hline PS1-10bkf & $08: 47: 18.713$ & $43: 24: 29.21$ & 03 & 55502 & $\cdots$ \\
\hline PS1-10bki & $08: 48: 33.834$ & $43: 59: 06.90$ & 03 & 55502 & $\ldots$ \\
\hline PS1-10blh & $02: 21: 46.162$ & $-05: 09: 05.76$ & 01 & 55510 & $\cdots$ \\
\hline PS1-10blp & 03:30:04.362 & $-28: 15: 24.09$ & 02 & 55507 & $\cdots$ \\
\hline PS1-10bls & $03: 32: 16.116$ & $-27: 11: 39.51$ & 02 & 55507 & $\ldots$ \\
\hline PS1-10bmb & $23: 29: 46.670$ & $00: 35: 25.82$ & 10 & 55507 & $\ldots$ \\
\hline PS1-10bmk & $03: 34: 30.852$ & $-27: 19: 14.09$ & 02 & 55524 & $\cdots$ \\
\hline PS1-10byj & $23: 33: 31.714$ & 00:08:24.06 & 10 & 55536 & LC incomplete \\
\hline PS1-10byn & $23: 25: 47.036$ & $00: 23: 46.68$ & 10 & 55536 & LC incomplete \\
\hline PS1-10byo & $23: 27: 55.950$ & $00: 20: 21.89$ & 10 & 55536 & LC incomplete \\
\hline PS1-10byq & $02: 19: 36.752$ & $-03: 31: 45.28$ & 01 & 55536 & $\cdots$ \\
\hline PS1-10byr & $22: 17: 50.801$ & 01:02:33.80 & 09 & 55536 & $\cdots$ \\
\hline PS1-10bys & $22: 17: 31.025$ & $01: 15: 19.48$ & 09 & 55536 & Fit not converged \\
\hline PS1-10bzo & $08: 48: 02.754$ & $44: 26: 57.43$ & 03 & 55546 & $\cdots$ \\
\hline PS1-10bzp & 08:40:03.150 & $44: 53: 39.61$ & 03 & 55546 & $\ldots$ \\
\hline PS1-10bzt & 08:48:59.894 & $44: 51: 56.45$ & 03 & 55546 & $\cdots$ \\
\hline PS1-10bzu & $08: 45: 25.506$ & 44:03:36.99 & 03 & 55546 & $\cdots$ \\
\hline PS1-10bzy & $03: 35: 22.742$ & $-27: 50: 25.00$ & 02 & 55546 & $\ldots$ \\
\hline PS1-10cad & $03: 34: 19.273$ & $-27: 34: 08.05$ & 02 & 55546 & $\ldots$ \\
\hline PS1-10cay & $03: 37: 19.804$ & $-26: 54: 06.29$ & 02 & 55546 & $\ldots$ \\
\hline PS1-10cbb & $03: 30: 51.155$ & $-26: 27: 35.94$ & 02 & 55546 & $\cdots$ \\
\hline PS1-10cbu & $08: 44: 16.542$ & $45: 20: 39.13$ & 03 & 55561 & $\ldots$ \\
\hline PS1-11e & $08: 35: 56.591$ & $43: 29: 40.24$ & 03 & 55561 & $\ldots$ \\
\hline PS1-11p & $09: 55: 10.067$ & 01:43:48.89 & 04 & 55566 & $\cdots$ \\
\hline PS1-11s & 10:03:09.364 & 02:08:36.19 & 04 & 55566 & $\cdots$ \\
\hline PS1-11t & $10: 02: 06.281$ & $02: 05: 16.06$ & 04 & 55566 & $\ldots$ \\
\hline PS1-11w & $10: 54: 43.784$ & $58: 37: 35.62$ & 05 & 55566 & $\ldots$ \\
\hline PS1-11aj & 10:03:16.274 & 03:28:29.81 & 04 & 55567 & $\ldots$ \\
\hline PS1-11ao & $02: 25: 44.073$ & $-03: 11: 46.36$ & 01 & 55568 & $|c|>0.25$ \\
\hline PS1-11at & 10:01:53.027 & $02: 02: 33.53$ & 04 & 55566 & $\ldots$ \\
\hline PS1-11bg & 10:03:58.861 & $02: 15: 44.71$ & 04 & 55572 & $\ldots$ \\
\hline PS1-11bh & 09:58:30.828 & $02: 52: 18.41$ & 04 & 55572 & $\cdots$ \\
\hline PS1-11bk & 09:56:19.761 & $01: 24: 34.08$ & 04 & 55572 & $\ldots$ \\
\hline PS1-11br & $03: 26: 43.681$ & $-27: 56: 10.45$ & 02 & 55576 & $\cdots$ \\
\hline PS1-11cn & $02: 23: 05.634$ & $-03: 36: 37.25$ & 01 & 55588 & $\cdots$ \\
\hline PS1-11co & $03: 28: 35.272$ & $-27: 20: 27.48$ & 02 & 55588 & $\ldots$ \\
\hline PS1-11fi & $14: 12: 57.070$ & $52: 44: 37.19$ & 07 & 55597 & $\cdots$ \\
\hline PS1-11gh & 03:38:00.402 & $-28: 00: 41.20$ & 02 & 55597 & LC incomplete \\
\hline PS1-11gr & 08:40:55.602 & $44: 47: 30.26$ & 03 & 55597 & LC incomplete \\
\hline PS1-11iv & $12: 14: 55.584$ & $46: 34: 33.26$ & 06 & 55597 & $\cdots$ \\
\hline PS1-11jo & $12: 16: 00.418$ & $47: 55: 13.49$ & 06 & 55597 & $\cdots$ \\
\hline PS1-11kk & $16: 10: 49.277$ & $55: 15: 05.31$ & 08 & 55602 & Initial cut \\
\hline PS1-11mq & $10: 49: 34.244$ & $57: 36: 56.63$ & 05 & 55633 & $\ldots$ \\
\hline PS1-11mz & $12: 14: 57.100$ & $46: 50: 42.15$ & 06 & 55633 & $\cdots$ \\
\hline PS1-11sk & $12: 12: 50.654$ & 47:08:44.75 & 06 & 55666 & $\cdots$ \\
\hline PS1-11um & $16: 14: 45.674$ & $56: 09: 35.89$ & 08 & 55675 & LC incomplete \\
\hline PS1-11uo & $10: 45: 24.781$ & $57: 13: 04.41$ & 05 & 55675 & $\ldots$ \\
\hline PS1-11uw & $16: 04: 56.287$ & $54: 52: 41.30$ & 08 & 55675 & $\cdots$ \\
\hline PS1-11vb & $10: 05: 28.755$ & 01:51:22.09 & 04 & 55675 & LC incomplete \\
\hline PS1-11wv & $12: 20: 33.010$ & $46: 39: 58.14$ & 06 & 55678 & $\cdots$ \\
\hline PS1-11xc & $16: 15: 51.680$ & $54: 26: 35.56$ & 08 & 55677 & $\cdots$ \\
\hline PS1-11xw & 12:19:54.009 & 48:08:25.00 & 06 & 55694 & $\cdots$ \\
\hline PS1-11yj & $16: 08: 30.469$ & $53: 41: 07.17$ & 08 & 55705 & $91 \mathrm{bg}$ \\
\hline
\end{tabular}


Table 4

(Continued)

\begin{tabular}{lrrrcc}
\hline \hline PS1 Name & R.A. (J2000) & Decl. (J2000) & MDF & \multicolumn{1}{c}{$t_{\text {disc }}$} & Cut \\
\hline PS1-11yr & $12: 26: 21.156$ & $46: 35: 45.33$ & 06 & 55705 & $\ldots$ \\
PS1-11yu & $16: 12: 46.179$ & $54: 52: 25.40$ & 08 & 55705 & Close to center \\
PS1-11zd & $14: 18: 40.107$ & $54: 10: 59.00$ & 07 & 55705 & $\ldots$ \\
PS1-11zg & $10: 53: 08.049$ & $57: 12: 04.55$ & 05 & 55708 & $\ldots$ \\
PS1-11zu & $16: 06: 28.184$ & $53: 52: 18.49$ & 08 & 55708 & $\ldots$ \\
PS1-11zv & $16: 11: 37.152$ & $53: 58: 12.96$ & 08 & 55708 & $\ldots$ \\
PS1-11zw & $16: 10: 25.814$ & $54: 08: 58.48$ & 08 & 55708 & $\ldots$ \\
PS1-11aaw & $14: 11: 30.070$ & $52: 28: 59.63$ & 07 & 55712 & $\left|x_{1}\right|>3.0$ \\
PS1-11abm & $12: 24: 43.960$ & $47: 40: 05.32$ & 06 & 55714 & $\ldots$ \\
PS1-11aea & $16: 14: 28.433$ & $54: 00: 10.88$ & 08 & 55740 & $\ldots$ \\
PS1-11aij & $16: 17: 16.520$ & $55: 22: 45.97$ & 08 & 55762 & $\ldots$ \\
PS1-11ajs & $22: 17: 50.984$ & $01: 28: 06.39$ & 09 & 55770 & $\ldots$ \\
PS1-11ala & $22: 12: 19.673$ & $01: 02: 09.41$ & 09 & 55782 & $\ldots$ \\
PS1-11alv & $22: 15: 52.038$ & $-00: 49: 30.57$ & 09 & 55795 & $\ldots$ \\
PS1-11ams & $22: 16: 32.270$ & $00: 28: 23.86$ & 09 & 55801 & Close to center \\
\hline
\end{tabular}

Notes. Summary of the general properties of the Pan-STARRS1 SNe Ia. The MDF fields are defined in Table 1 . The discovery date $t_{\text {disc }}$ is given in MJD. The cuts are explained in Section 7.2-cuts 7-10 in Section 7.2 are referred to as "LC incomplete."

Table 5

Photometric Calibration Error Budget

\begin{tabular}{lcc}
\hline \hline Source & $\begin{array}{c}\text { Uncertainty } \\
\text { (Millimag) }\end{array}$ & Section \\
\hline Photometric non-linearity & 1 & 5.3 .1 \\
Centroid accuracy & $\lesssim 1$ & Appendix D \\
Forced photometry & 2 & 5.3 .2 \\
Baseline flux correction & $\lesssim 1$ & 5.3 .4 \\
Instr. response function & 3 & 6.1 \\
Flux determination & 5 & 6.1 \\
Net offset wrt Calspec & $8,5,6,3$ & 3.2 in S 14 \\
Zero point propagation & 3 & 6.1 \\
Spatial variation & $5,6,4,6$ & 6.2 \\
Temporal variation & 3 & 6.2 \\
\hline Total internal PS1 & $\sim 12$ & 3.2 in S 14 \\
\hline SED conformity to AB & $5-15$ &
\end{tabular}

Notes. The dominant systematic uncertainties in defining the Pan-STARRS1 photometric system. If four numbers are given, they refer to griz $\mathrm{P}_{1}$, respectively. We assign a range of uncertainty to the SED conformity to the AB system, since the color dependence is not easily enumerated and is discussed in more detail in S14. The bandpass uncertainties is included into the instrumental response function and also discussed in S14.

\subsubsection{Forced Photometry}

For each SN, we calculate the weighted average position and then perform forced photometry in every difference image at this position. Because we convolve the template, the PSF shape in each difference image is the same as the one in the original image and is kept unchanged. The only free parameter is the peak value of the PSF.

The success of this photometry method strongly depends on the accuracy of the WCS solution. If the WCS accuracy is poor, the forced position is off the true peak and the flux is underestimated. This effect is strongest for high-S/N measurements. In Figure 5, we show the magnitude difference $\Delta m$ between the forced photometry and photometry where the centroid of the SN is determined in each individual image versus the $\mathrm{S} / \mathrm{N}$ of the forced photometry. At high $\mathrm{S} / \mathrm{N}$, the average magnitude difference is an insignificant $0.5 \mathrm{mmag}$ different from zero. Though

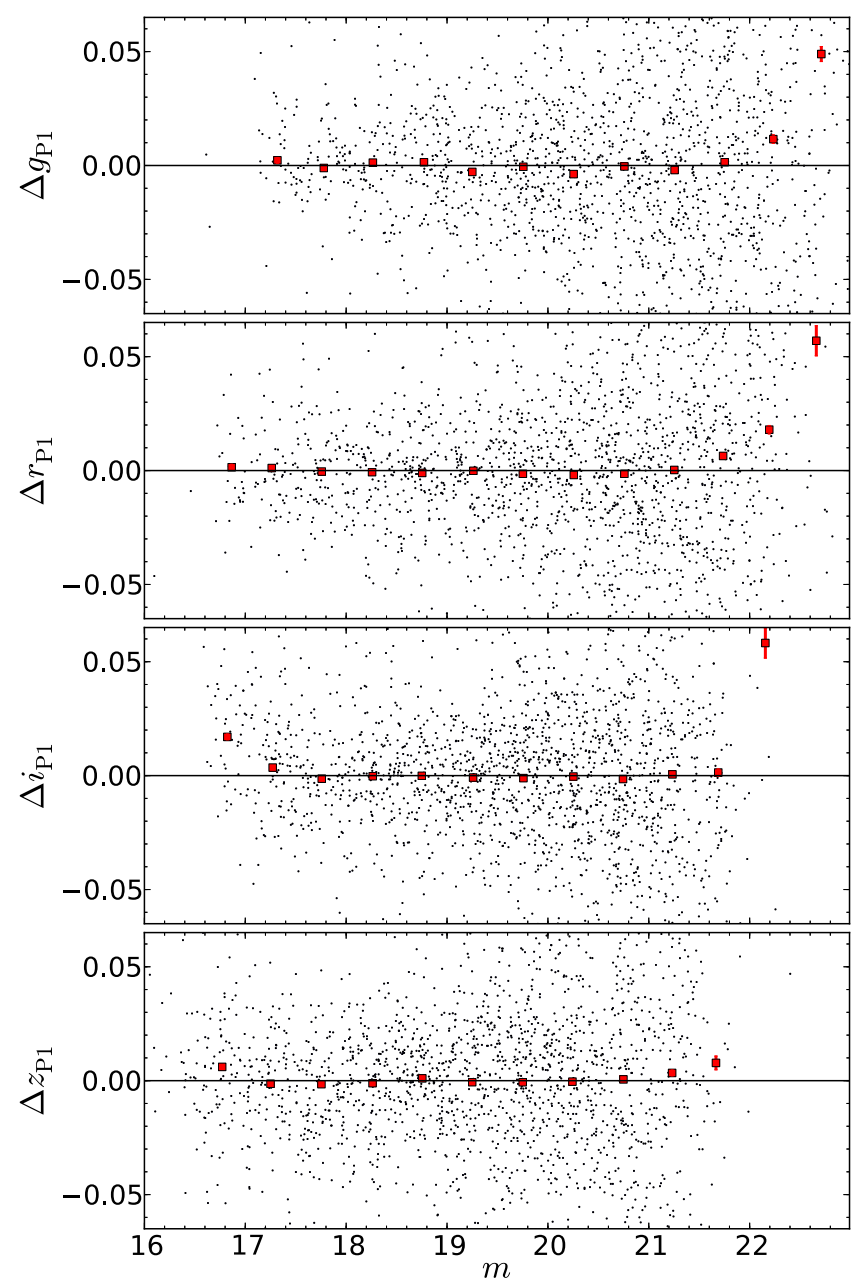

Figure 3. Comparison between deep and nightly stack photometry for stars in the MDFs. The red circles indicate the weighted average of the magnitude difference $\Delta m=m_{\text {deep }}-m_{\text {nightly }}$ from 100 randomly selected images for each of rrizP1 $_{\mathrm{P} 1}$ from top to bottom, respectively. They are consistent with zero within the errors at all magnitudes except at the very brightest and faintest ends. In particular, $i_{\mathrm{P} 1}$ and $z_{\mathrm{P} 1}$ show significant deviation from zero for $m<17$ mag. Malmquist bias is likely the cause of the deviation at faint magnitudes. The black dots show a small subset of $\Delta m$ values for illustration.

(A color version of this figure is available in the online journal.)

there is some scatter in the magnitude differences, the scatter is small compared to the Poisson noise. This makes sense because the same data are used with both methods. The important result is that for high $\mathrm{S} / \mathrm{N}$, the scatter is small, symmetric, and shows no significant bias. If there are any problems with the astrometric calibration, centroiding, or differences between forced and unforced PSF fitting, systematic differences would occur. In Appendix D, we quantify the photometric bias introduced by centroiding uncertainties, and calculate the expectation value of this bias for each SN depending on its centroid uncertainty (see Figure 21). Since the uncertainty in the centroid is larger at higher redshift, there is a redshift dependence of this bias of $\sim 2$ mmag from low to high redshift. We correct the light curves for this photometric bias and estimate that the residual photometric bias is less than $1 \mathrm{mmag}$ (see Table 5). Another advantage of forced photometry is that it does not suffer from a photometric Malmquist bias at low S/N. This effect can be seen in Figure 5. At $\mathrm{S} / \mathrm{N}=10$ and 3 , the regular photometry is on average brighter by $0.5 \%$ and $2.5 \%$, respectively. 


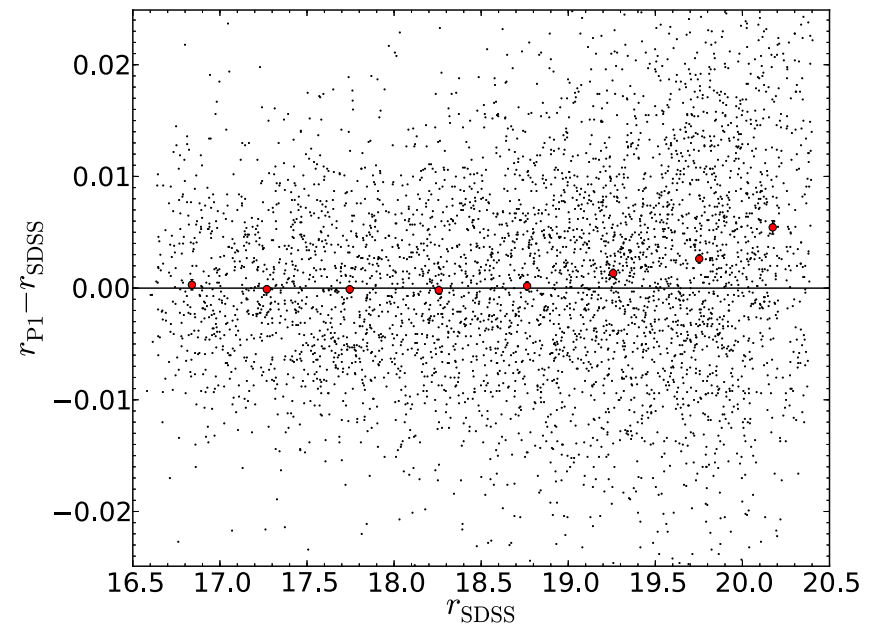

Figure 4. Comparison between $r_{\mathrm{P} 1}$ and $r_{\mathrm{SDSS}}$, where $r_{\mathrm{SDSS}}$ is the SDSS $r$ band magnitude converted into Pan-STARRS1 natural system magnitudes using Equation (6) and Table 6 from T12b. The red circles indicate the average of the magnitude difference.

(A color version of this figure is available in the online journal.)

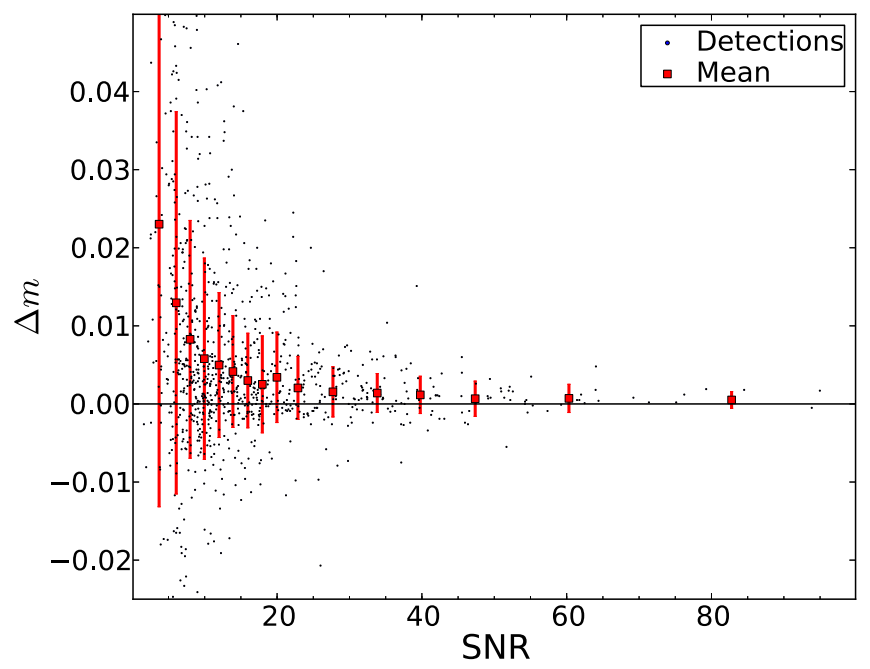

Figure 5. Comparison of forced and regular photometry for PS1 SNe Ia as a function of $\mathrm{S} / \mathrm{N}$. The red circles indicate the weighted average of the magnitude difference $\Delta m=m_{\text {forced }}-m_{\text {regular }}$ of SNe detections for different $\mathrm{S} / \mathrm{N}$ bins. The error bars indicate the standard deviation. The black dots show a small subset of $\Delta m$ values for illustration. At high $\mathrm{S} / \mathrm{N}$, the average magnitude difference is an insignificant $0.5 \mathrm{mmag}$ different from zero, indicating that there are no issues with the astrometric calibration, centroiding, or differences between forced and unforced PSF fitting.

(A color version of this figure is available in the online journal.)

There is additional uncertainty since we perform forced photometry on the $\mathrm{SNe}$, but "regular" photometry (i.e., free $x, y$ positions) on our field stars from which we determine the zero point. We have chosen reference stars for our photometric calibration that have a $S / N \geqslant 20$. For such stars, the difference between regular and forced photometry is smaller than $2 \mathrm{mmag}$ (see Figure 5). This has been added to our systematic error table (see Table 5). A technique to mitigate this effect is to do forced photometry for both the field stars and $\mathrm{SNe}$ (e.g., Astier et al. 2006).

\subsubsection{Empirical Adjustment of Uncertainties}

In our pipeline, the variance terms are propagated through all of the image processing. However, this underestimates the true

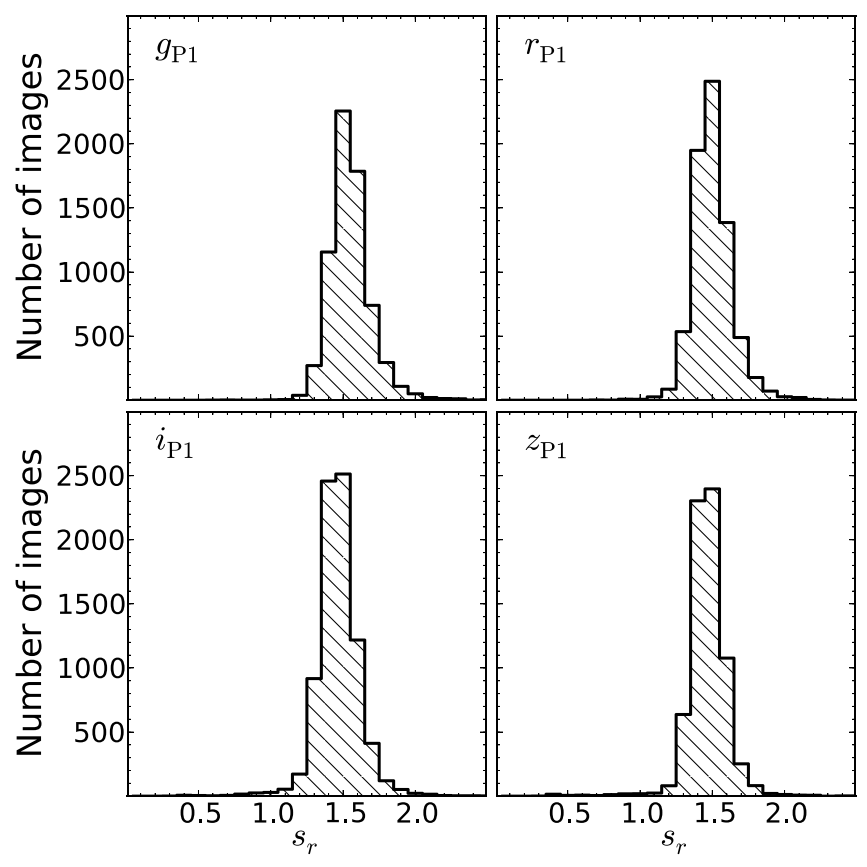

Figure 6. Histogram of the empirical multiplicative factor $s_{r}$, which is used to correct the flux uncertainties of a given difference image.

uncertainties in the measurements since the resampling process as well as kernel-matching one image to the other introduces covariance between the pixels. In order to empirically determine by how much the uncertainties are underestimated, we measure the flux $f_{r}$ and its uncertainty $\sigma_{r}$ at random positions in a given difference image in exactly the same way as we measure the SN flux. We calculate the weighted mean of the flux measurements $\bar{f}_{r}$, excluding outliers by a $3 \sigma$. This $\bar{f}_{r}$ is an empirical estimate of the systematic bias in the photometry at any position for that particular difference image, and we correct all photometry of that image with $\bar{f}_{r}$. This value is typically on the order of $\sim$ three ADU. We then estimate the factor $s_{r}$ by which the uncertainties are underestimated using the reduced chi-square $s_{r}=\sqrt{\chi_{r}^{2}}$, as described in more detail in Appendix C. Figure 6 shows the histograms of $s_{r}$ for all difference images and filters used for the $\mathrm{SNe}$ light curves. The histograms for griz $_{\mathrm{P} 1}$ are nearly identical with a peak at $s \sim 1.5$, with the bluer filters having slightly higher values since on average their larger PSF leads to larger convolution kernels and thus to more covariance. We empirically correct the difference image uncertainties by multiplying them with $s_{r}$. Note that this is a multiplicative correction since the ratio of covariance to variance is independent of the flux level in a given pixel. In Section 5.3.4 we show that the baseline (pre-SN) flux uncertainties are in general very good representations of the true uncertainties, since their reduced chi-square distribution has a peak at 1.0. A similar approach of empirically adjusting the uncertainties has been applied to the SNLS data (Astier et al. 2006). An alternative approach is to apply "scene modeling" (Holtzman et al. 2008), which fits a scene that includes the SN and a model of its environment to the data in the original, unwarped image, thus avoiding the complication of correlated pixels.

\subsubsection{Baseline Flux Adjustment}

We construct the templates on the assumption that there is no SN flux present. However, stacking images is not a perfect process and there may be some small biases in the 


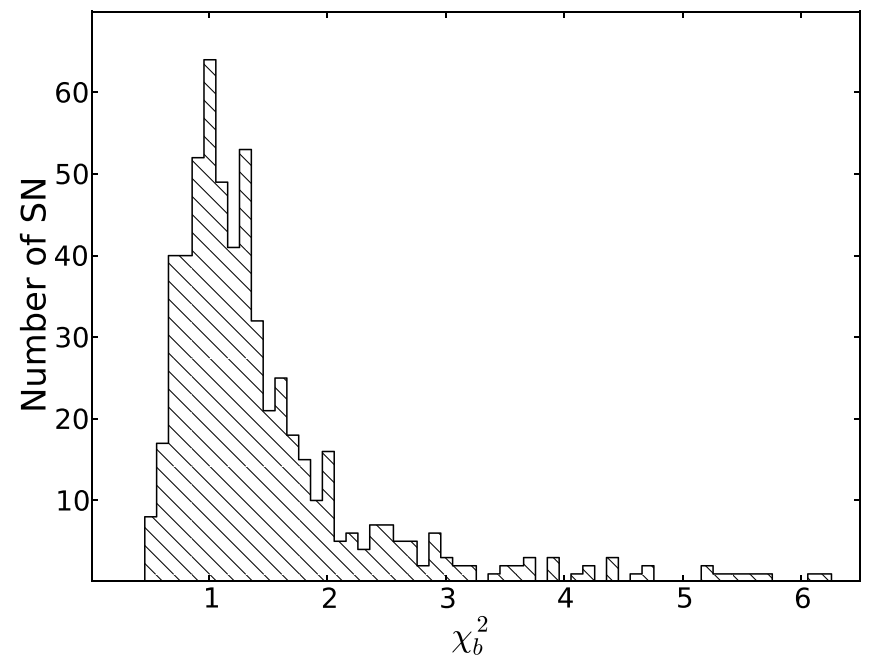

Figure 7. Histogram of the baseline flux reduced chi-square $\chi_{b}^{2}$. Note that the peak of the distribution is very close to unity, indicating that the difference image uncertainties are a good representation of the true errors. The tail toward large $\chi_{\text {norm }}^{2}$ is most likely due to difference image artifacts due to bright host galaxies in close proximity to the $\mathrm{SNe}$.

template construction (e.g., SN flux included in some images, or artifacts from different PSF sizes). Since we always subtract the same template from each image, this single realization of noise creates the same systematic offset in every difference image measurement of a given $\mathrm{SN}$ and directly affects the peak magnitude of the SN. We therefore correct all the light curves by the average baseline flux calculated from the fluxes where there is known to be no SN flux, as described below. This correction is equivalent to creating a deep stack with all available images, but in addition, it corrects for any imperfection in the template stack creation and photometry.

We note that even after the baseline flux adjustment, there is still some small Poisson uncertainty left since a finite number of measurements were used. However, this uncertainty is very small compared to the uncertainty in a single epoch, and it does not bias the light curve fits in a significant way.

We calculate the weighted average of all forced photometry difference image measurements at epochs that are $<-30$ days or $>200$ days from maximum for each SN, and subtract this baseline flux from all SNe light curve measurements. We find that the calculated offsets are on average zero. However, the standard deviation of the distribution of these offsets is 24 ADUs for a zero point of 30.0. The significance of correcting with these offsets depends on the magnitude of the SNe. For example, 24 ADUs correspond to 3 and $41 \mathrm{mmag}$ to $\mathrm{SNe}$ of magnitude 19 and 23, respectively. Thus, this correction is non-negligible for an individual SN, particularly for faint ones. However, since the baseline flux correction distribution is symmetric and centered around zero, we find that any systematic bias possibly introduced to the peak magnitude can only be very small, and we assign an upper limit of $1 \mathrm{mmag}$ to it (see Table 5).

The normalized $\chi_{b}^{2}$ from the baseline flux calculation is shown in Figure 7. The peak of the distribution is very close to one, indicating that our measured uncertainties are generally good estimates of the true uncertainties. There is a tail toward large $\chi_{b}^{2}$, which we have determined is due to difference image artifacts introduced by bright extended sources near the position of the $\mathrm{SN}$. This effect is particularly strong at the center of bright sources, and the outliers in the $\chi_{b}^{2}$ are exclusively SNe Ia close to the nucleus of a bright galaxy.
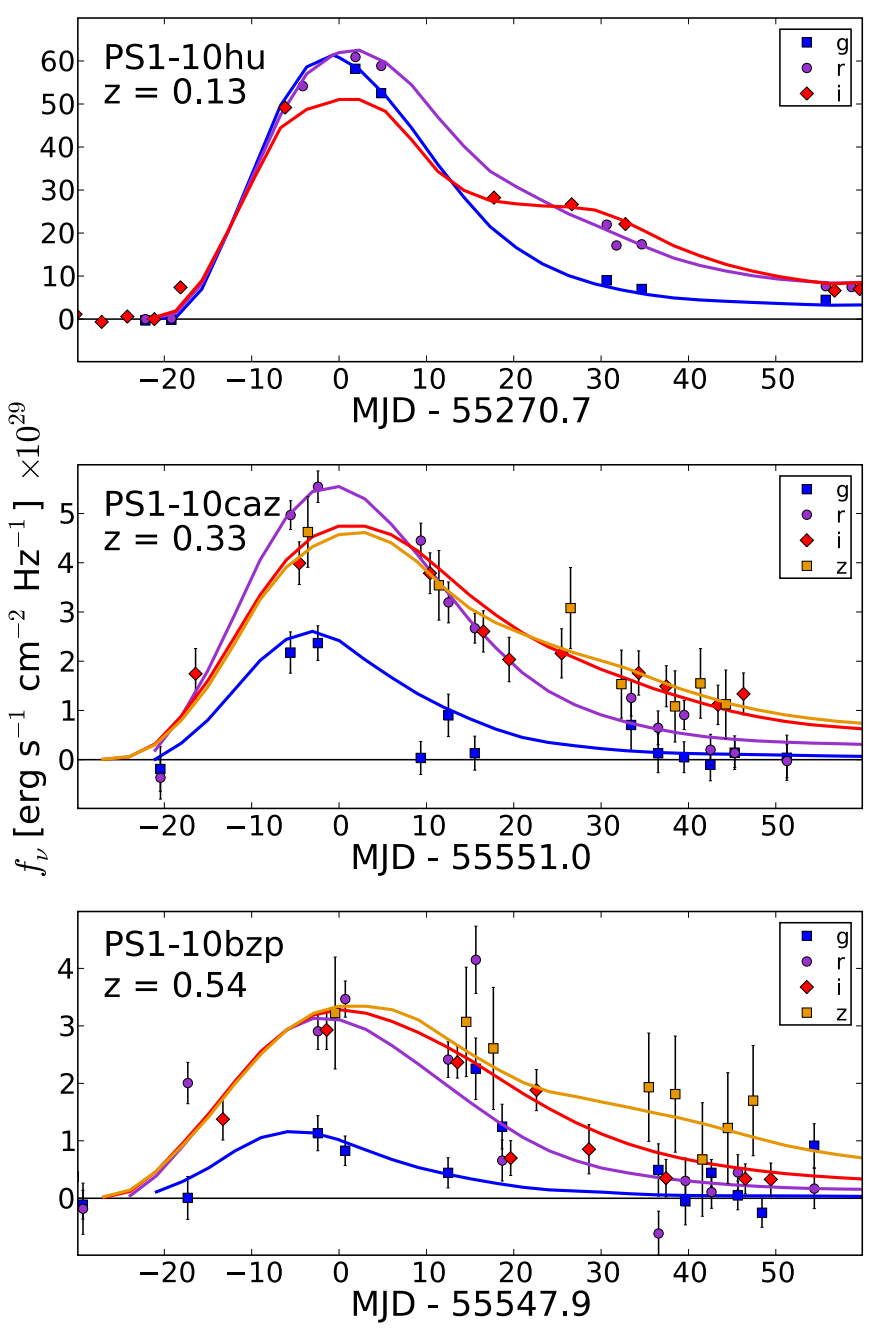

Figure 8. Example light curves for three Pan-STARRS1 SNe Ia at different redshifts. MJD offset are applied so that zero is the time of maximum in restframe B. The SALT2 fits are shown with the solid lines.

(A color version of this figure is available in the online journal.)

Since these artifacts have a net flux of zero, they do not introduce a systematic bias into the photometry, but the large background variation near these artifacts artificially increases $\chi_{b}^{2}$. This effect reduces the quality of the light curve fits for some $\mathrm{SNe}$, which do not pass our quality cuts for further analysis. Future analyses that seek the largest useful sample should address this problem.

In Figure 8 we show representative PS1 SNe Ia light curves: PS1-10hu, PS1-10caz, and PS1-10bzp with redshifts of 0.13, 0.33 , and 0.64 from top to bottom, respectively. The solid lines are their respective light curve fits as described in Section 7. The light curves are available in machine-readable format in the electronic edition. As an abbreviated example, we show the light curve of SN PS1-10hu in Table 6.

\section{PHOTOMETRIC CALIBRATION}

As Sullivan et al. (2011) has pointed out, our ability to constrain the properties of dark energy from SNe Ia observations is limited by systematic errors from the photometric calibration. The unique area and depth of our SN survey creates the opportunity to observe $\mathrm{SNe}$ over a wide redshift range with the same telescope and filters. This improves the potential for minimizing calibration errors. 
Table 6

Light Curve of PS1-10hu

\begin{tabular}{|c|c|c|c|c|}
\hline Name & MJD & Filter & Flux $f$ & Magnitude $m$ \\
\hline PS1-10hu & 55230.60229 & $g$ & $20.0 \pm 21.5$ & $\cdots$ \\
\hline PS1-10hu & 55233.53140 & $g$ & $-5.8 \pm 24.8$ & $\cdots$ \\
\hline PS1-10hu & 55236.59489 & $g$ & $-0.9 \pm 15.4$ & $\cdots$ \\
\hline PS1-10hu & 55248.52526 & $g$ & $-1.4 \pm 7.8$ & $\cdots$ \\
\hline PS1-10hu & 55272.56808 & $g$ & $1550.3 \pm 25.6$ & $19.52 \pm 0.02$ \\
\hline PS1-10hu & 55275.51514 & $g$ & $1387.7 \pm 34.2$ & $19.64 \pm 0.02$ \\
\hline PS1-10hu & 55326.41381 & $g$ & $119.6 \pm 20.2$ & $22.31 \pm 0.17$ \\
\hline PS1-10hu & 55230.61622 & $r$ & $7.2 \pm 20.6$ & $\ldots$ \\
\hline PS1-10hu & 55233.54702 & $r$ & $-32.2 \pm 28.7$ & $\cdots$ \\
\hline PS1-10hu & 55236.60945 & $r$ & $18.3 \pm 13.7$ & $\cdots$ \\
\hline PS1-10hu & 55248.53908 & $r$ & $-3.9 \pm 8.4$ & $\cdots$ \\
\hline PS1-10hu & 55251.52352 & $r$ & $34.9 \pm 32.9$ & $\cdots$ \\
\hline PS1-10hu & 55266.55484 & $r$ & $1487.1 \pm 36.2$ & $19.57 \pm 0.02$ \\
\hline PS1-10hu & 55272.58426 & $r$ & $1652.1 \pm 25.6$ & $19.45 \pm 0.02$ \\
\hline PS1-10hu & 55275.52937 & $r$ & $1614.2 \pm 98.5$ & $19.48 \pm 0.07$ \\
\hline PS1-10hu & 55305.35085 & $r$ & $471.3 \pm 20.1$ & $20.82 \pm 0.04$ \\
\hline PS1-10hu & 55326.42714 & $r$ & $221.3 \pm 31.0$ & $21.64 \pm 0.14$ \\
\hline PS1-10hu & 55332.32633 & $r$ & $202.3 \pm 10.8$ & $21.73 \pm 0.05$ \\
\hline PS1-10hu & 55231.58320 & $i$ & $9.8 \pm 13.7$ & $\cdots$ \\
\hline PS1-10hu & 55234.55779 & $i$ & $-16.7 \pm 13.4$ & $\cdots$ \\
\hline PS1-10hu & 55237.57200 & $i$ & $-15.3 \pm 12.1$ & $\cdots$ \\
\hline PS1-10hu & 55240.53452 & $i$ & $20.3 \pm 11.1$ & $\ldots$ \\
\hline PS1-10hu & 55243.53133 & $i$ & $-5.7 \pm 23.1$ & $\ldots$ \\
\hline PS1-10hu & 55246.46399 & $i$ & $18.1 \pm 15.7$ & $\cdots$ \\
\hline PS1-10hu & 55249.55923 & $i$ & $-9.7 \pm 11.0$ & $\cdots$ \\
\hline PS1-10hu & 55252.55308 & $i$ & $178.6 \pm 32.6$ & $21.87 \pm 0.18$ \\
\hline PS1-10hu & 55288.44303 & $i$ & $771.3 \pm 25.1$ & $20.28 \pm 0.03$ \\
\hline PS1-10hu & 55297.34091 & $i$ & $709.2 \pm 20.4$ & $20.37 \pm 0.03$ \\
\hline PS1-10hu & 55303.48521 & $i$ & $585.3 \pm 17.4$ & $20.58 \pm 0.03$ \\
\hline PS1-10hu & 55327.42882 & $i$ & $185.2 \pm 28.8$ & $21.83 \pm 0.16$ \\
\hline PS1-10hu & 55330.30423 & $i$ & $185.8 \pm 12.9$ & $21.83 \pm 0.07$ \\
\hline PS1-10hu & 55339.28203 & $i$ & $283.2 \pm 56.7$ & $21.37 \pm 0.20$ \\
\hline PS1-10hu & 55348.34014 & $i$ & $126.0 \pm 19.1$ & $22.25 \pm 0.15$ \\
\hline PS1-10hu & 55235.58945 & $z$ & $16.3 \pm 21.6$ & $\cdots$ \\
\hline PS1-10hu & 55238.56725 & $z$ & $0.2 \pm 16.4$ & $\cdots$ \\
\hline PS1-10hu & 55241.49175 & $z$ & $10.3 \pm 25.7$ & $\cdots$ \\
\hline PS1-10hu & 55247.53664 & $z$ & $-24.9 \pm 16.5$ & $\cdots$ \\
\hline PS1-10hu & 55250.57895 & $z$ & $0.7 \pm 16.0$ & $\cdots$ \\
\hline PS1-10hu & 55268.58466 & $z$ & $865.0 \pm 33.8$ & $20.16 \pm 0.04$ \\
\hline PS1-10hu & 55280.35431 & $z$ & $548.8 \pm 14.8$ & $20.65 \pm 0.03$ \\
\hline PS1-10hu & 55298.39193 & $z$ & $572.0 \pm 23.1$ & $20.61 \pm 0.04$ \\
\hline PS1-10hu & 55304.31987 & $z$ & $548.5 \pm 18.1$ & $20.65 \pm 0.03$ \\
\hline PS1-10hu & 55322.38723 & $z$ & $254.3 \pm 18.1$ & $21.49 \pm 0.07$ \\
\hline PS1-10hu & 55325.36617 & $z$ & $171.4 \pm 16.9$ & $21.92 \pm 0.10$ \\
\hline PS1-10hu & 55334.33428 & $z$ & $186.9 \pm 11.0$ & $21.82 \pm 0.06$ \\
\hline PS1-10hu & 55340.28431 & $z$ & $203.3 \pm 36.0$ & $21.73 \pm 0.18$ \\
\hline
\end{tabular}

Notes. An abbreviated example PS1 light curve-PS1-10hu. The light curves of all $\mathrm{SNe}$ in the cosmological sample are available in machine-readable format in the electronic edition. Only photometry within 40 days before and 100 days after maximum at MJD $=55270$ is presented here. All fluxes $f$ are with respect to a zero point of 27.5, and the magnitudes are accordingly calculated as $m=$ $-2.5 \log 10(\mathrm{f})+27.5$. We only show magnitudes $m$ for fluxes with at least $3 \sigma$ significance.

(This table is available in its entirety in a machine-readable form in the online journal. A portion is shown here for guidance regarding its form and content.)

Tonry et al. (2012b) measures the system passbands, including both the instrumental sensitivity and atmospheric transmission functions. The PS1 photometric system is fundamentally based on HST Calspec spectrophotometric observations. For seven Calspec standards, Tonry et al. (2012b) determines slight adjustments to the bandpasses so that the photometry of the standards best agrees with the spectrophotometry of the standards with HST spectra. The fields with Calspec standards are passed to Schlafly et al. (2012), which performs the ubercal calibration that ties the observations of stars in these fields with stars across the entire $3 \pi$ survey. The zero points set by Tonry et al. (2012b) are mostly preserved in this stage, though may be slightly adjusted so that there is optimal relative calibration across the sky. The MDFs are calibrated in this process. S14 then checks this process by analyzing the photometry of all the Calspec standards observed in the full $3 \pi$ survey and comparing the photometry to the synthetic photometry of the positions. S14 finds the $\mathrm{AB}$ offsets so that the photometry and spectrophotometry agree. The offsets found here are applied to all of the MDF photometry and SNe light curves.

The total systematic uncertainty in the PS1 calibration may be broken down into four parts: the instrumental response function, the propagation of zero points across the MDFs, the adherence of the PS1 zero points to the AB system, and spatial/temporal variation in the photometric calibration. The uncertainty of the instrumental response function is given in Tonry et al. (2012b, T12b hereafter). This analysis also details the full systematic uncertainties in the PS1 implementation of the $\mathrm{AB}$ system, though we have redone a significant amount of the error accounting. The uncertainty in the propagation of zero points across the sky must be included as there are 10 MDFs with large separations in distance. We use the results of Schlafly et al. (2012) to correct for this. The adherence of the PS1 calibration to a true AB system is analyzed in detail by the companion paper S14. Finally, the spatial and temporal variation of the instrumental response function is analyzed in this paper.

Our entire systematic error budget for calibration is summarized in Table 5. We find that after accounting for the full systematic error budget, the overall systematic uncertainty for each filter is $\sim 0.012$ mag without including the uncertainty in the HST Calspec definition of the AB system. We briefly review the uncertainties that are discussed in external work, and analyze the remaining uncertainties below.

\subsection{Absolute Calibration and All-sky Photometry}

The system's response function naturally divides into the instrumental response function (mirrors, optics, filters, and detectors) and the atmospheric extinction. In principle, it is possible to determine these two components independently without the reliance on standard stars: the atmospheric extinction can be determined with measurements and modeling, while the instrumental response function can be traced with a high-accuracy NIST-calibrated photodiode in combination with a tunable laser system (Stubbs \& Tonry 2006; Stubbs et al. 2010). In practice, however, the calibration accuracy is improved by including observations of spectrophotometric standards, which provide the overall normalization of the photometric system and can verify the derived system and atmospheric response functions. T12b describes in detail how the PS1 photometric system has been determined with this recipe.

The Calspec standards, and thus also the PS1 photometry, are fundamentally based on models of hydrogen white dwarf atmospheres and the absolute flux for Vega in the $V$ band (Bohlin 2007). Substantial work has been invested in improving the HST spectrophotometric standards (e.g., Colina \& Bohlin 1994; Bohlin 1996; Bohlin et al. 2001), and they have the distinct advantage that the Calspec standard star observations are not marred by the atmosphere. However, even in space, 


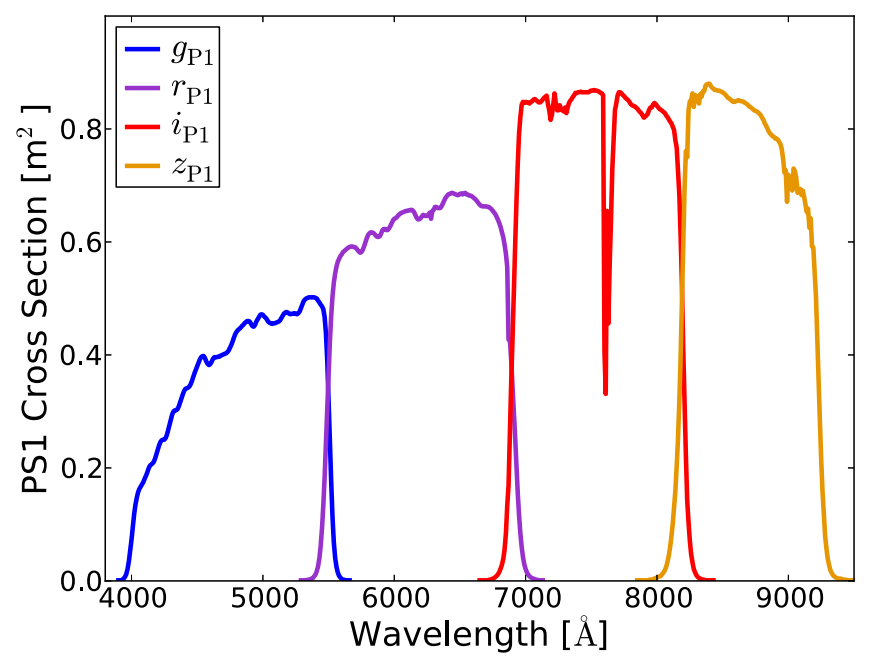

Figure 9. System response function for the four Pan-STARRS1 filters griz $_{\mathrm{P} 1}$ at an air mass of 1.2, assuming representative precipitable water vapor and aerosol exponent parameters of $0.65 \mathrm{~cm}$ and 0.7 , respectively (T12b).

(A color version of this figure is available in the online journal.)

complications like charge transfer efficiencies, non-linearity of detectors, and secular degradation of the optics and detectors contribute to systematic measurement biases (Bohlin 2007). Combining all this, we adopt a systematic error of 5-15 mmag from non-conformity of the HST Calspec standards to the AB system, depending on wavelength (Bohlin \& Gilliland 2004). This wavelength dependence is further discussed in S14.

The PS1 survey observed seven standard stars (1740346, KF01T5, KF06T2, KF08T3, LDS749B, P177D, and WD1657343) on MJD 55744 (UT 2011 July 2) in photometric conditions at a wide range of air masses in all filters. The stars were placed on the same CCD detector/amplifier, which we chose to be away from the center of the field, where spatial variation in the PSF adds additional uncertainty to photometric measurements as shown in Section 6.2. In theory, a common normalization to all filters should be sufficient to match the observations to the synthetic magnitudes. However, the synthetic colors of the standard stars deviate from the observed colors. T12b attempts to correct for these differences by correcting the instrument response function. For the filters gri $_{\mathrm{P} 1}$, they determine the corrections to be $0.012,0.019,0.009$, and $-0.009 \mathrm{mag}$, respectively. The correction is small because the agreement between the in situ NIST-based response function and the manufacturer's benchmark measurements of the filters and the CCD is very good. The system response function (instrument response function and atmosphere) of the PS1 photometric system for grizP1 is shown in Figure 9 at an air mass of 1.2.

The photometric normalization in the limited set of fields with the Calspec standards is then propagated across the sky, encompassing all of the Medium Deep survey fields, using the same übercalibration method (Padmanabhan et al. 2008) that has been successfully used for the SDSS All-sky Survey. The details of this übercalibration are described in Schlafly et al. (2012). Comparing the PS1 and SDSS photometry, they find that both surveys show similar photometric calibration errors. They estimate that the relative precision in $g_{\mathrm{P} 1}, r_{\mathrm{P} 1}$, and $i_{\mathrm{P} 1}$ is $<10 \mathrm{mmag}$, and $\sim 10 \mathrm{mmag}$ in $z_{\mathrm{P} 1}$. Since we have $10 \mathrm{MDFs}$ distributed over the full sky, we estimate that the uncertainty introduced by zero point variation is $10 / \sqrt{10}$ mmag. S14 repeat the process outlined in $\mathrm{T} 12 \mathrm{~b}$ of adjusting the filter transmissions based on agreement between observed and synthetic photometry. They increase the number of Calspec standards used to 10. S14 determines that systematic uncertainties in the photometry of the standard stars are no larger than $5 \mathrm{mmag}$. They also find that small adjustments $\Delta$ griz $_{\mathrm{P} 1}=[-0.008,-0.0095,-0.004,-0.007]$ should be added to the zero points defined by $\mathrm{T} 12 \mathrm{~b}$ and Schlafly et al. (2012). This improves the measurement of the offset for each filter, and reduces the systematic uncertainty due to the adjustments from 10 mmag to those given in Table 5 ("net offset wrt Calspec").

To test the calibration, T12b finds an excellent agreement between the stellar locus constructed from observed PS1 photometry from a field with very low Galactic extinctions with its synthetic colors, with systematic differences of $\sim 1 \%$. They also transform SDSS stripe $82 \mathrm{mag}$ into the PS1 photometric system, compare them to the observed PS1 magnitudes, and find differences at the 1\%-2\% level. T12b also notes that there are differences at the same level between the different SDSS data releases. A more detailed exploration of the source of these differences is given in S14.

\subsection{Spatial and Time Variation of the Pan-STARRS1 Instrumental Response Function}

The system response function can vary with wavelength, position on the focal plane, and time. This section quantifies the systematic biases introduced by errors in the determination of the instrument throughput.

For PS1, the spatial variation of the instrument response function is removed by flat-fielding and the on-sky illumination correction, which is constructed using dithered images of a dense stellar field (Magnier 2006). However, Stubbs et al. (2010) have suggested that after these corrections there may still be a residual spatial variation in the photometry that is band dependent. To test this, we compared PS1 photometry of stars in three MDFs against SDSS photometry of the same stars converted to the PS1 system of T12b (see S14 for further details). Figure 10 shows the results of this test, plotting the median difference between the PS1 and SDSS photometry. The most striking disagreement is in the first bin, which lies at the center of the focal plane where the optics of the telescope generate strong spatial variation of the PSF. Because the central region is so difficult to handle, we discard from our cosmological analysis the three SNe Ia that fall in the central region (PS1-11ams, PS110f, and PS1-11yu) because we cannot be certain they meet our threshold for photometric fidelity.

Across the rest of the focal plane, the PS1 photometry in each filter exhibits a steady constant offset relative to SDSS, ranging from $0.5 \%$ in $r_{\mathrm{P} 1}$ to $3 \%$ in $z_{\mathrm{P} 1}$. These overall differences between the synthetic and observed PS 1 magnitudes are known and described in more detail in T12b and S14. They can be taken as conservative upper limits on the systematic biases in the PS1 photometry. After removing this offset, the remaining difference between SDSS and PS1 photometry is less than $1 \%$ in each filter and that is further reduced to $\sim 5 \mathrm{mmag}$ for all filters when systematic shifts between the MDFs are accounted for. ${ }^{24}$ We therefore use $5 \mathrm{mmag}$ as the limit for spatial consistency in our error budget (Table 5). The differences between PS1 and SDSS shown here are most likely due to flat-fielding issues, as the expected differences due to variations in the filter passbands

\footnotetext{
${ }^{24}$ We attribute the MDF-to-MDF variation to large-scale spatial variations in the SDSS photometric system (Schlafly \& Finkbeiner 2011; Schlafly et al. 2012).
} 
Table 7

Fit Parameters of low- $z$ Sample

\begin{tabular}{|c|c|c|c|c|c|c|c|c|c|}
\hline SN & Set & $z_{\mathrm{cmb}}$ & $t_{\text {peak }}$ & $P_{\text {fit }}$ & $x_{1}$ & $c$ & $m_{B}$ & $\mu$ & $E(B-V)$ \\
\hline 19900 & JRK07 & $0.031(0.001)$ & $48076.88(0.59)$ & 0.9960 & $0.476(0.231)$ & $-0.054(0.036)$ & $15.993(0.065)$ & $35.587(0.166)$ & $0.075(0.012)$ \\
\hline 1990af & JRK07 & $0.050(0.001)$ & $48196.03(0.27)$ & .0000 & $-2.114(0.235)$ & $-0.038(0.048)$ & $17.508(0.039)$ & $36.689(0.190)$ & $0.028(0.005)$ \\
\hline $1992 \mathrm{P}$ & JRK07 & $0.026(0.001)$ & $48719.90(0.50)$ & 0.4810 & $0.069(0.352)$ & $-0.083(0.045)$ & $15.811(0.036)$ & $35.441(0.195)$ & $0.017(0.003)$ \\
\hline 1992ae & JRK07 & $0.075(0.001)$ & $48804.90(0.66)$ & 0.7470 & $-0.708(0.186)$ & $-0.029(0.055)$ & $18.216(0.052)$ & $37.565(0.199)$ & $0.029(0.005)$ \\
\hline 1992aq & JRK07 & $0.101(0.001)$ & $48834.37(0.69)$ & 1.0000 & $-1.326(0.264)$ & $-0.071(0.063)$ & $19.065(0.053)$ & $38.460(0.215)$ & $0.010(0.002)$ \\
\hline 1992al & JRK07 & $0.014(0.001)$ & 48838.84(0.17) & 1.0000 & $-0.222(0.110)$ & $-0.110(0.029)$ & $14.204(0.036)$ & $33.880(0.198)$ & $0.027(0.004)$ \\
\hline $1992 b c$ & JRK07 & $0.021(0.001)$ & $48913.16(0.08)$ & 0.3520 & $0.782(0.100)$ & $-0.121(0.027)$ & $14.847(0.032)$ & $34.700(0.169)$ & $0.018(0.003)$ \\
\hline $1992 \mathrm{bh}$ & JRK07 & $0.042(0.001)$ & $48921.06(0.57)$ & 0.9960 & $-0.053(0.182)$ & $0.042(0.047)$ & $17.363(0.038)$ & $36.575(0.188)$ & $0.018(0.003)$ \\
\hline $1992 b l$ & JRK07 & $0.042(0.001)$ & $48947.68(0.48)$ & 0.9640 & $-1.631(0.138)$ & $-0.045(0.051)$ & $17.070(0.050)$ & $36.342(0.196)$ & $0.009(0.001)$ \\
\hline $1992 b p$ & JRK07 & $0.079(0.001)$ & $48980.87(0.33)$ & 0.9480 & $-0.882(0.163)$ & $-0.102(0.052)$ & $18.075(0.049)$ & $37.631(0.194)$ & $0.056(0.009)$ \\
\hline $1992 \mathrm{bs}$ & JRK07 & $0.063(0.001)$ & $48986.08(0.58)$ & 0.9680 & $-0.230(0.166)$ & $-0.034(0.049)$ & $18.046(0.045)$ & $37.478(0.185)$ & $0.009(0.001)$ \\
\hline 1992bo & JRK07 & $0.019(0.001)$ & $48986.62(0.10)$ & 1.0000 & $-1.983(0.112)$ & $-0.050(0.031)$ & $15.501(0.036)$ & $34.739(0.177)$ & $0.022(0.003)$ \\
\hline 1993B & JRK07 & $0.071(0.001)$ & $49006.04(0.74)$ & 0.0621 & $-0.395(0.196)$ & $0.069(0.057)$ & $18.265(0.069)$ & $37.345(0.197)$ & $0.064(0.010)$ \\
\hline $1993 \mathrm{H}$ & JRK07 & $0.025(0.001)$ & $49067.70(0.39)$ & 0.9770 & $-1.972(0.096)$ & $0.092(0.032)$ & $16.417(0.052)$ & $35.202(0.165)$ & $0.049(0.008)$ \\
\hline 19930 & JRK07 & $0.052(0.001)$ & $49134.68(0.16)$ & 0.7920 & $-0.563(0.138)$ & $-0.077(0.046)$ & $17.383(0.045)$ & $36.905(0.181)$ & $0.043(0.007)$ \\
\hline 1993ag & JRK07 & $0.049(0.001)$ & $49317.30(0.33)$ & 0.8030 & $-0.784(0.154)$ & $0.082(0.051)$ & $17.635(0.067)$ & $36.618(0.192)$ & $0.083(0.013)$ \\
\hline $1994 S$ & JRK07 & $0.016(0.001)$ & $49518.66(0.31)$ & 0.5680 & $0.287(0.229)$ & $-0.082(0.030)$ & $14.504(0.037)$ & $34.160(0.188)$ & $0.017(0.003)$ \\
\hline $1995 \mathrm{ac}$ & JRK07 & $0.049(0.001)$ & 49993.54(0.17) & 0.6400 & $0.724(0.119)$ & $-0.048(0.028)$ & $16.807(0.037)$ & $36.417(0.149)$ & $0.034(0.005)$ \\
\hline $1995 \mathrm{ak}$ & JRK07 & $0.022(0.001)$ & $50022.45(0.51)$ & 0.2310 & $-1.071(0.168)$ & $0.070(0.036)$ & $15.775(0.052)$ & $34.757(0.172)$ & $0.031(0.005)$ \\
\hline $1996 \mathrm{C}$ & JRK07 & $0.028(0.001)$ & $50129.51(0.47)$ & 0.9300 & $0.510(0.167)$ & $0.049(0.030)$ & $16.362(0.038)$ & $35.631(0.164)$ & $0.011(0.002)$ \\
\hline $1996 \mathrm{ab}$ & JRK07 & $0.124(0.001)$ & $50223.37(1.36)$ & 0.8210 & $0.074(0.436)$ & $-0.179(0.057)$ & $19.291(0.060)$ & $39.231(0.220)$ & $0.026(0.004)$ \\
\hline 1996bl & JRK07 & $0.035(0.001)$ & $50377.10(0.24)$ & 0.8580 & $-0.071(0.153)$ & $-0.002(0$. & $16.472(0.0$ & & $0.074(0.012)$ \\
\hline 1997E & JRK07 & $0.013(0.001)$ & $50468.54(0.20)$ & 1.0000 & $-1.634(0.169)$ & $0.028(0.034)$ & $14.915(0.072)$ & $33.953(0.204)$ & $0.092(0.015)$ \\
\hline $1997 \mathrm{Y}$ & JRK07 & $0.017(0.001)$ & $50488.76(1.03)$ & 0.9920 & $-0.930(0.270)$ & $0.001(0.032)$ & $15.080(0.037)$ & $34.302(0.190)$ & $0.014(0.002)$ \\
\hline $1997 \mathrm{dg}$ & JRK07 & $0.030(0.001)$ & $50721.01(0.44)$ & 0.8980 & $-0.394(0.225)$ & $-0.020(0.034)$ & $16.618(0$. & 35.982 & 0.06 \\
\hline 1997do & JRK07 & $0.011(0.001)$ & $50766.87(0.16)$ & 0.0903 & $0.244(0.221)$ & $0.070(0$. & $14.103(C$ & 33.269 & 0.05 \\
\hline $1998 \mathrm{~V}$ & JRK07 & $0.017(0.001)$ & $86(0.35)$ & 0.5220 & -0.278 & $0.029(0$ & 14.983(0. & $34205 x$ & 0.14 \\
\hline $1998 \mathrm{ab}$ & JRK07 & $0.028(0.001)$ & $50914.77(0.11)$ & 0.0078 & 0.258 & $0.103(0$. & 3960 & & 002) \\
\hline 1998bp & JRK07 & $0.011(0.001)$ & $50935.42(0.46)$ & 0.2480 & $-2.471(0.185)$ & $0.188(0.037)$ & $15.044(0.061)$ & $33.450(0.235)$ & $0.061(0.010)$ \\
\hline 1998co & JRK07 & $0.017(0.001)$ & $50987.72(1.81)$ & 0.9980 & $-2.997(2.869)$ & $0.029(0.060)$ & $15.376(0.098)$ & $34.218(0.410)$ & $0.035(0.006)$ \\
\hline $1998 d x$ & JRK07 & $0.054(0.001)$ & $51072.60(0.65)$ & 0.9760 & $-1.678(0.395)$ & $-0.099(0.040)$ & $17.305(0.054)$ & $36.742(0.168)$ & $0.033(0.005)$ \\
\hline $1998 \mathrm{eg}$ & JRK07 & $0.024(0.001)$ & $51111.49(0.91)$ & 0.9950 & $-0.561(0.416)$ & $0.017(0.037)$ & $15.935(0$ & $35.156(0.182)$ & $0.091(0.015)$ \\
\hline $1998 \mathrm{ef}$ & JRK07 & $0.018(0.001)$ & $51114.24(0.18)$ & 0.9520 & $-1.048(0.240)$ & $-0.095(0.034)$ & $14.569(0.059)$ & $34.081(0.183)$ & $0.059(0.009)$ \\
\hline 1999aw & JRK07 & $0.039(0.001)$ & $51255.11(0.33)$ & 0.2000 & $2.246(0.179)$ & $-0.084(0.029)$ & $16.490(0.0$ & $36.427(0.1$ & $0.026(0.004)$ \\
\hline $1999 \mathrm{cc}$ & JRK07 & $0.032(0.001)$ & $51315.96(0.28)$ & 1.0000 & $-1.567(0.152)$ & $-0.011(0.028)$ & $16.520(0.032)$ & $35.692(0.156)$ & $0.019(0.003)$ \\
\hline $1999 \mathrm{cp}$ & JRK07 & $0.011(0.001)$ & $51364.08(0.16)$ & 0.6690 & $0.339(0.199)$ & $-0.084(0.031)$ & $13.688(0.037)$ & $33.360(0.231)$ & $0.019(0.003)$ \\
\hline $1999 \mathrm{dq}$ & JRK07 & 0.013 & 51436.6 & 1.0 & 0.849 & 0. & 14.191 & 33 & 0.08 \\
\hline 1999ee & JRK07 & $0.011(0.001)$ & $51470.18(0.04)$ & 1.0000 & $0.797(C$ & 0.239 & 14.611 & 33312 & 0.01 \\
\hline 1999ek & JRK07 & $0.018(0.001)$ & $51482.76(0.14)$ & 1.0000 & $-0.677(0.106)$ & $0.159(0.0$ & $15.703(0.242)$ & $456(0.200)$ & $0.415(0.066)$ \\
\hline 1999gp & JRK07 & $0.027(0.001)$ & $51551.39(0.07)$ & 1.0000 & $1.583(0.082)$ & $0.014(0.028)$ & $15.786(0.042)$ & $35.319(0.158)$ & $0.045(0.007)$ \\
\hline 2000ca & JRK07 & $0.023(0.001)$ & $51666.66(0.24)$ & 0.9450 & $0.479(0.127)$ & $-0.102(0.029)$ & $15.325(0.049)$ & $35.073(0.165)$ & $0.054(0.009)$ \\
\hline $2000 \mathrm{cf}$ & JRK07 & $0.037(0.001)$ & $51673.41(0.39)$ & 0.9330 & $-0.485(0.110)$ & $-0.021(0.030)$ & $16.815(0.041)$ & $36.168(0.152)$ & $0.026(0.004)$ \\
\hline $2000 \mathrm{cn}$ & JRK07 & $0.024(0.001)$ & $51707.91(0.12)$ & 0.9940 & $-2.376(0.256)$ & $0.086(0.032)$ & $16.299(0.047)$ & $35.046(0.168)$ & $0.046(0.007)$ \\
\hline $2000 \mathrm{dk}$ & JRK07 & $0.018(0.001)$ & $51812.89(0.18)$ & 0.9990 & $-1.906(0.171)$ & $-0.020(0.033)$ & $15.115(0.051)$ & $34.269(0.183)$ & $0.057(0.009)$ \\
\hline $2000 \mathrm{fa}$ & JRK07 & $0.022(0.001)$ & $51893.07(0.12)$ & 0.9620 & $0.496(0.147)$ & $0.048(0.032)$ & $15.652(0.053)$ & $34.923(0.172)$ & $0.056(0.009)$ \\
\hline $2001 \mathrm{~V}$ & JRK07 & $0.017(0.001)$ & $51974.28(0.06)$ & 1.0000 & $0.854(0.101)$ & $-0.020(0.027)$ & $14.319(0.032)$ & $33.858(0.257)$ & $0.016(0.003)$ \\
\hline $2001 \mathrm{ba}$ & JRK07 & $0.030(0.001)$ & $52034.83(0.20)$ & 0.7880 & $0.111(0.121)$ & $-0.181(0.041)$ & $15.932(0.047)$ & $35.883(0.179)$ & $0.052(0.008)$ \\
\hline $2001 \mathrm{cn}$ & JRK07 & $0.015(0.001)$ & $52073.56(0.30)$ & 1.0000 & $-0.584(0.080)$ & $0.131(0.029)$ & $15.036(0.047)$ & $33.890(0.186)$ & $0.048(0.008)$ \\
\hline $2001 \mathrm{cz}$ & JRK07 & $0.015(0.001)$ & $52104.51(0.15)$ & 0.9980 & $0.085(0.112)$ & $0.048(0.031)$ & $14.807(0.061)$ & $34.019(0.188)$ & $0.086(0.014)$ \\
\hline $2001 \mathrm{G}$ & CFA3 & $0.018(0.001)$ & $51931.25(1.43)$ & 0.9120 & $0.163(0.480)$ & $-0.042(0.044)$ & $14.710(0.052)$ & $34.223(0.198)$ & $0.013(0.002)$ \\
\hline $2001 \mathrm{~V}$ & CFA3 & $0.016(0.001)$ & $51973.81(0.08)$ & 0.9990 & $0.855(0.125)$ & $-0.008(0.028)$ & $14.312(0.034)$ & $33.811(0.249)$ & $0.016(0.003)$ \\
\hline 2001ah & CFA3 & $0.059(0.001)$ & $52006.90(0.97)$ & 0.8110 & $1.134(0.731)$ & $-0.033(0.033)$ & $17.385(0.073)$ & $37.004(0.214)$ & $0.011(0.002)$ \\
\hline 2001ay & CFA3 & $0.031(0.001)$ & $52025.61(0.64)$ & 0.0821 & $2.971(0.310)$ & $0.088(0.035)$ & $16.487(0.044)$ & $35.977(0.166)$ & $0.015(0.002)$ \\
\hline $2001 \mathrm{az}$ & CFA3 & $0.040(0.001)$ & $52030.96(1.33)$ & 1.0000 & $1.173(0.709)$ & $-0.082(0.035)$ & $16.672(0.060)$ & $36.454(0.162)$ & $0.048(0.008)$ \\
\hline $2001 b f$ & CFA3 & $0.015(0.001)$ & $52046.87(0.47)$ & 0.2710 & $0.583(0.157)$ & $0.001(0.033)$ & $14.504(0.067)$ & $33.938(0.185)$ & $0.079(0.013)$ \\
\hline $2001 \mathrm{eh}$ & CFA3 & $0.037(0.001)$ & $52170.11(0.62)$ & 0.6380 & $1.614(0.232)$ & $-0.028(0.032)$ & $16.383(0.048)$ & $36.054(0.154)$ & $0.050(0.008)$ \\
\hline 2001ep & CFA3 & $0.013(0.001)$ & $52200.24(0.18)$ & 0.9270 & $-0.908(0.105)$ & $0.081(0.029)$ & $14.650(0.042)$ & $33.619(0.195)$ & $0.039(0.006)$ \\
\hline $2001 \mathrm{fe}$ & CFA3 & $0.015(0.001)$ & $52229.24(0.46)$ & 0.9980 & $0.561(0.155)$ & $-0.022(0.029)$ & $14.422(0.035)$ & $33.927(0.189)$ & $0.018(0.003)$ \\
\hline $2002 \mathrm{G}$ & CFA3 & $0.035(0.001)$ & $52300.13(0.60)$ & 0.5760 & $-1.401(0.524)$ & $0.203(0.050)$ & $17.312(0.081)$ & $35.822(0.181)$ & $0.010(0.002)$ \\
\hline $2002 \mathrm{cr}$ & CFA3 & $0.010(0.001)$ & $52408.90(0.08)$ & 0.9750 & $-0.463(0.120)$ & $-0.020(0.029)$ & $13.944(0.038)$ & $33.300(0.227)$ & $0.019(0.003)$ \\
\hline 2002de & CFA3 & $0.028(0.001)$ & $52433.96(0.33)$ & 0.9990 & $0.548(0.644)$ & $0.111(0.033)$ & $16.415(0.038)$ & $35.492(0.165)$ & $0.015(0.002)$ \\
\hline 2002dp & CFA3 & $0.011(0.001)$ & $52451.16(0.24)$ & 0.9830 & $-0.086(0.323)$ & $0.093(0.033)$ & $14.340(0.046)$ & $33.385(0.220)$ & $0.040(0.006)$ \\
\hline $2002 \mathrm{dj}$ & CFA3 & $0.010(0.001)$ & $52451.46(0.16)$ & 0.9760 & $0.120(0.204)$ & $0.074(0.031)$ & $13.716(0.063)$ & $32.852(0.224)$ & $0.078(0.012)$ \\
\hline 2002ha & CFA3 & $0.014(0.001)$ & $52581.41(0.20)$ & 1.0000 & $-1.334(0.137)$ & $-0.062(0.035)$ & $14.469(0.065)$ & $33.836(0.195)$ & $0.079(0.013)$ \\
\hline 2002he & CFA3 & $0.025(0.001)$ & $52586.17(0.16)$ & 1.0000 & $-1.677(0.267)$ & $-0.044(0.041)$ & 15.992(0.061) & $35.254(0.162)$ & $0.032(0.005)$ \\
\hline 2002hu & CFA3 & $0.038(0.001)$ & $52592.48(0.19)$ & 0.9940 & $0.409(0.178)$ & $-0.073(0.029)$ & $16.379(0.040)$ & $36.024(0.144)$ & $0.036(0.006)$ \\
\hline
\end{tabular}


Table 7

(Continued)

\begin{tabular}{|c|c|c|c|c|c|c|c|c|c|}
\hline SN & Set & $z_{\mathrm{cmb}}$ & $t_{\text {peak }}$ & $P_{\text {fit }}$ & $x_{1}$ & $c$ & $m_{B}$ & $\mu$ & $E(B-V)$ \\
\hline 2002jy & CFA3 & $0.022(0.001)$ & $52634.50(0.52)$ & 0.9950 & $0.787(0.193)$ & $-0.034(0.031)$ & $15.486(0.047)$ & $35.059(0.166)$ & $0.044(0.007)$ \\
\hline $2002 \mathrm{kf}$ & CFA3 & $.020(0.001)$ & $52640.23(0.43)$ & 0.9630 & $-1.124(0.117)$ & $-0.013(0.034)$ & $15.501(0.070)$ & $34.741(0.168)$ & $0.086(0.014)$ \\
\hline $2003 \mathrm{U}$ & CFA3 & $.028(0.001)$ & $52677.57(0.22)$ & 0.9960 & $-1.985(0.549)$ & $-0.035(0.047)$ & $16.213(0.061)$ & $35.401(0.171)$ & $0.024(0.004)$ \\
\hline $2003 \mathrm{ch}$ & CFA3 & $0.030(0.001)$ & $52726.30(0.42)$ & 0.9340 & $-1.264(0.215)$ & $-0.031(0.034)$ & $16.438(0.054)$ & $35.717(0.159)$ & $0.057(0.009)$ \\
\hline $2003 \mathrm{cq}$ & CFA3 & $0.034(0.001)$ & $52739.77(0.22)$ & 0.1830 & $-0.713(0.203)$ & $0.091(0.059)$ & $16.904(0.069)$ & $35.869(0.184)$ & $0.016(0.003)$ \\
\hline $2003 \mathrm{fa}$ & CFA3 & $0.039(0.001)$ & $52807.40(0.11)$ & 0.9600 & $1.408(0.172)$ & $-0.031(0.029)$ & $16.466(0.040)$ & $36.116(0.143)$ & $0.032(0.005)$ \\
\hline $2003 i c$ & CFA3 & $0.054(0.001)$ & $52907.08(1.17)$ & 0.9870 & $-1.796(0.332)$ & $-0.018(0.045)$ & $17.378(0.068)$ & $36.538(0.166)$ & $0.031(0.005)$ \\
\hline 2003iv & CFA3 & $0.035(0.001)$ & $52934.29(0.54)$ & 0.9980 & $-2.023(0.316)$ & $-0.054(0.042)$ & $16.812(0.077)$ & $36.057(0.165)$ & $0.098(0.016)$ \\
\hline 2003 it & CFA3 & $0.024(0.001)$ & $52935.27(0.53)$ & 0.7290 & $-1.524(0.244)$ & $0.043(0.037)$ & $16.127(0.054)$ & $35.132(0.170)$ & $0.055(0.009)$ \\
\hline $2003 \mathrm{kc}$ & CFA3 & $0.035(0.001)$ & $52979.25(0.68)$ & 0.0011 & $-0.708(0.225)$ & $0.111(0.045)$ & $16.901(0.065)$ & $35.803(0.160)$ & $0.017(0.003)$ \\
\hline 2004as & CFA3 & $0.033(0.001)$ & $53086.26(0.22)$ & 0.8210 & $0.358(0.195)$ & $0.023(0.032)$ & $16.682(0.036)$ & $36.013(0.152)$ & $0.012(0.002)$ \\
\hline $2004 \mathrm{bg}$ & CFA3 & $0.022(0.001)$ & $53110.07(0.70)$ & 0.1120 & $0.462(0.168)$ & $-0.024(0.032)$ & $15.391(0.054)$ & $34.887(0.160)$ & $0.018(0.003)$ \\
\hline $2004 \mathrm{ef}$ & CFA3 & $0.030(0.001)$ & $53265.04(0.80)$ & 0.9980 & $-1.671(0.307)$ & $0.088(0.043)$ & $16.654(0.053)$ & $35.492(0.246)$ & $0.044(0.007)$ \\
\hline $2005 \mathrm{M}$ & CFA3 & $0.026(0.001)$ & $53402.76(1.77)$ & 0.0748 & $1.893(0.707)$ & $0.170(0.066)$ & $15.894(0.083)$ & $34.970(0.308)$ & $0.026(0.004)$ \\
\hline $2005 \mathrm{am}$ & CFA3 & $0.010(0.001)$ & $53436.51(0.29)$ & 0.9970 & $-1.741(0.069)$ & $0.046(0.030)$ & $13.394(0.046)$ & $32.357(0.231)$ & $0.044(0.007)$ \\
\hline $2005 \mathrm{el}$ & CFA3 & $0.015(0.001)$ & $53646.66(0.11)$ & 0.9370 & $-1.242(0.060)$ & $-0.070(0.031)$ & $14.671(0.066)$ & $34.076(0.287)$ & $0.084(0.013)$ \\
\hline 2005 eq & CFA3 & $0.029(0.001)$ & $53654.70(0.16)$ & 1.0000 & $1.208(0.130)$ & $-0.017(0.030)$ & $16.032(0.0$ & $35.611(0.2$ & $0.058(0.009)$ \\
\hline $2005 \mathrm{eu}$ & CFA3 & $0.035(0.001)$ & $53660.70(0.14)$ & 0.1520 & $0.903(0.126)$ & $-0.068(0.036)$ & $16.258(0.076)$ & $35.955(0.147)$ & $0.100(0.016)$ \\
\hline $2005 \mathrm{hc}$ & CFA3 & $0.045(0.001)$ & $53668.36(0.19)$ & 0.9450 & $0.409(0.143)$ & $-0.024(0.029)$ & 17.114(0.034) & $36.604(0.202)$ & $0.026(0.004)$ \\
\hline 2005hj & CFA3 & $0.057(0.001)$ & $53673.28(1.07)$ & 0.7920 & $2.303(0.495)$ & $-0.060(0.040)$ & $17.454(0.054)$ & $37.326(0.247)$ & $0.032(0.005)$ \\
\hline 2005ir & CFA3 & $0.075(0.001)$ & $53683.45(1.05)$ & 0.6880 & $2.994(0.788)$ & $-0.043(0.046)$ & $18.247(0.055)$ & $38.157(0.295)$ & $0.025(0.004)$ \\
\hline $2005 \mathrm{iq}$ & CFA3 & $0.034(0.001)$ & $53687.83(0.19)$ & 1.0000 & $-1.147(0.152)$ & $-0.087(0.029)$ & $16.558(0.033)$ & $36.031(0.209)$ & $0.017(0.003)$ \\
\hline $2005 \mathrm{kc}$ & CFA3 & $0.015(0.001)$ & $53697.83(0.11)$ & 0.0018 & $-0.328(0.098)$ & $0.242(0.034)$ & $15.441(0.075)$ & $33.976(0.260)$ & $0.097(0.016)$ \\
\hline $2005 \mathrm{ki}$ & CFA3 & $0.020(0.001)$ & $53705.44(0.13)$ & 0.9910 & $-1.636(0.112)$ & $-0.075(0.034)$ & $15.327(0.041)$ & $34.694(0.239)$ & $0.026(0.004)$ \\
\hline $2005 \mathrm{mc}$ & CFA3 & $0.026(0.001)$ & $53733.95(0.31)$ & 0.6290 & $-2.469(0.179)$ & $0.238(0.036)$ & $17.013(0.045)$ & $35.261(0.231)$ & $0.036(0.006)$ \\
\hline $20051 \mathrm{z}$ & CFA3 & $0.041(0.001)$ & $53736.06(0.21)$ & 0.0874 & $-1.018(0.164)$ & $0.064(0.035)$ & $17.384(0.060)$ & $36.392(0.151)$ & $0.014)$ \\
\hline 2005 na & CFA3 & $0.027(0.001)$ & $53741.67(0.54)$ & 0.7100 & $-0.310(0.127)$ & $-0.018(0.035)$ & $15.859(0.0$ & $35.228(0.219)$ & $0.062(0.010)$ \\
\hline $2005 \mathrm{~ms}$ & CFA3 & $0.027(0.001)$ & $53744.16(0.11)$ & 0.9620 & $0.334(0.125)$ & $-0.050(0.0$ & $15.876(0.0$ & $35.437(0$ & $0.027(0.004)$ \\
\hline 2006D & CFA3 & $0.010(0.001)$ & $53757.48(0.14)$ & 1.0000 & $-1.496(0.100)$ & $-0.002(0.0$ & $13.854(0.0$ & & $0.037(0.006)$ \\
\hline $2006 \mathrm{~N}$ & CFA3 & $0.014(0.001)$ & $53761.04(0.29)$ & 0.7650 & $-2.008(0.117)$ & $-0.022(0.033)$ & $14.869(0.064)$ & $34.013(0.189)$ & $0.078(0.012)$ \\
\hline $2006 \mathrm{~S}$ & CFA3 & $0.033(0.001)$ & $53770.16(0.17)$ & 0.1660 & $0.856(0.110)$ & $0.049(0.028)$ & $16.596(0.031)$ & $35.915(0.147)$ & $0.014(0.002)$ \\
\hline $2006 \mathrm{ac}$ & CFA3 & $0.024(0.001)$ & $53781.88(0.14)$ & 0.9170 & $-0.994(0.099)$ & $0.039(0.029)$ & $15.897(0.033)$ & $34.988(0.155)$ & $0.013(0.002)$ \\
\hline 2006an & CFA3 & $0.065(0.001)$ & $53790.55(0.04)$ & 0.0011 & $0.338(0.230)$ & $-0.044(0.033)$ & $17.818(0.049)$ & $37.361(0.153)$ & $0.022(0.003)$ \\
\hline $2006 a z$ & CFA3 & $0.032(0.001)$ & $53826.97(0.13)$ & 0.5090 & $-1.423(0.070)$ & $-0.074(0.028)$ & $16.227(0.030)$ & $35.619(0.147)$ & $0.012(0.002)$ \\
\hline 2006ax & CFA3 & $0.017(0.001)$ & $53827.49(0.06)$ & 0.9990 & $0.270(0.066)$ & $-0.081(0.028)$ & $14.796(0.041)$ & $34.449(0.241)$ & $0.041(0.007)$ \\
\hline $2006 \mathrm{bt}$ & CFA3 & $0.032(0.001)$ & $53858.36(0.20)$ & 0.1530 & $0.127(0.107)$ & $0.097(0.028)$ & $16.678(0.040)$ & $35.742(0.146)$ & $0.041(0.007)$ \\
\hline $2006 \mathrm{cf}$ & CFA3 & $0.042(0.001)$ & $53875.05(0.86)$ & 0.2870 & $-0.609(0.331)$ & $-0.097(0.042)$ & $16.820(0.055)$ & $36.400(0.164)$ & $0.013(0.002)$ \\
\hline 2006cj & CFA3 & $0.068(0.001)$ & $53875.92(1.40)$ & 1.0000 & $2.546(0.919)$ & $-0.099(0.0$ & $17.876(0.042)$ & $37.904(0.185)$ & $0.002)$ \\
\hline $2006 \mathrm{cq}$ & CFA3 & $0.049(0.001)$ & $53889.95(0.51)$ & 0.9460 & $0.196(0.5$ & $0.005(0$. & $17.325(0.035)$ & $36.693(0.157)$ & $0.013(0.002)$ \\
\hline 2006сp & CFA3 & $0.023(0.001)$ & $53897.70(0.09)$ & 0.9700 & $0.290(0.111)$ & $0.056(0.030)$ & $15.711(0.037)$ & $34.927(0.160)$ & $0.023(0.004)$ \\
\hline $2006 c z$ & CFA3 & $0.043(0.001)$ & $53907.02(1.58)$ & 0.0429 & $1.613(0.710)$ & $0.103(0.056)$ & $755(0.070)$ & $36.007(0.245)$ & $0.081(0.013)$ \\
\hline $2006 \mathrm{et}$ & CFA3 & $0.022(0.001)$ & $53994.52(0.33)$ & 0.9880 & $0.693(0.192)$ & $0.130(0.032)$ & $15.704(0.040)$ & $34.740(0.232)$ & $0.015(0.002)$ \\
\hline $2006 \mathrm{gr}$ & CFA3 & $0.034(0.001)$ & $54013.36(0.10)$ & 0.8150 & $0.814(0.108)$ & $0.060(0.030)$ & $16.716(0.056)$ & $35.992(0.146)$ & $0.069(0.011)$ \\
\hline $2006 \mathrm{kf}$ & CFA3 & $0.021(0.001)$ & $54041.32(0.31)$ & 0.9740 & $-2.181(0.135)$ & $-0.052(0.046)$ & $15.724(0.123)$ & $34.941(0.237)$ & $0.180(0.029)$ \\
\hline $2006 \mathrm{mo}$ & CFA3 & $0.037(0.001)$ & $54047.86(0.41)$ & 0.9700 & $-2.110(0.172)$ & $0.037(0.038)$ & $17.181(0.053)$ & $36.123(0.158)$ & $0.036(0.006)$ \\
\hline 2006le & CFA3 & $0.017(0.001)$ & $54048.46(0.06)$ & 1.0000 & $0.839(0.077)$ & $-0.058(0.057)$ & $14.766(0.189)$ & $34.423(0.168)$ & $0.305(0.049)$ \\
\hline 2006mp & CFA3 & $0.023(0.001)$ & $54054.31(0.18)$ & 0.9890 & $0.921(0.253)$ & $0.005(0.031)$ & $15.729(0.038)$ & $35.198(0.163)$ & $0.029(0.005)$ \\
\hline $2006 \mathrm{ob}$ & CFA3 & $0.059(0.001)$ & $54063.04(0.23)$ & 0.8430 & $-2.215(0.214)$ & $-0.019(0.035)$ & $17.975(0.038)$ & $37.081(0.214)$ & $0.026(0.004)$ \\
\hline 2006on & CFA3 & $0.068(0.001)$ & $54064.14(0.84)$ & 0.9450 & $0.829(0.798)$ & $0.101(0.056)$ & $18.280(0.090)$ & $37.428(0.188)$ & $0.104(0.017)$ \\
\hline $20060 \mathrm{a}$ & CFA3 & $0.059(0.001)$ & $54067.05(0.19)$ & 0.8930 & $1.152(0.315)$ & $-0.008(0.032)$ & $17.633(0.040)$ & $37.173(0.145)$ & $0.032(0.005)$ \\
\hline 2006qo & CFA3 & $0.029(0.001)$ & $54083.00(0.09)$ & 0.9450 & $0.337(0.093)$ & $0.173(0.029)$ & $16.581(0.039)$ & $35.430(0.149)$ & $0.032(0.005)$ \\
\hline $2006 s r$ & CFA3 & $0.024(0.001)$ & $54092.89(0.20)$ & 0.9760 & $-1.311(0.162)$ & $-0.009(0.033)$ & $15.895(0.061)$ & $35.095(0.159)$ & $0.075(0.012)$ \\
\hline 2006te & CFA3 & $0.032(0.001)$ & $54097.37(0.51)$ & 0.8750 & $-0.159(0.130)$ & $-0.078(0.034)$ & $16.288(0.051)$ & $35.871(0.153)$ & $0.037(0.006)$ \\
\hline 2006td & CFA3 & $0.016(0.001)$ & $54098.92(0.47)$ & 1.0000 & $-1.335(0.181)$ & $0.101(0.033)$ & $15.477(0.062)$ & $34.322(0.181)$ & $0.071(0.011)$ \\
\hline $2007 \mathrm{~F}$ & CFA3 & $0.025(0.001)$ & $54123.97(0.08)$ & 1.0000 & $0.549(0.073)$ & $-0.050(0.027)$ & $15.631(0.030)$ & $35.222(0.154)$ & $0.013(0.002)$ \\
\hline 2007R & CFA3 & $0.031(0.001)$ & $54129.49(0.51)$ & 0.0978 & $-1.247(0.132)$ & $-0.083(0.035)$ & $16.453(0.059)$ & $35.898(0.159)$ & $0.038(0.006)$ \\
\hline 2007ai & CFA3 & $0.032(0.001)$ & $54173.83(0.55)$ & 0.2080 & $1.261(0.297)$ & $0.208(0.054)$ & $16.841(0.159)$ & $35.706(0.241)$ & $0.246(0.039)$ \\
\hline 2007au & CFA3 & $0.020(0.001)$ & $54184.23(0.16)$ & 0.4100 & $-2.818(0.1$ & $0.188(0.035)$ & & $34.670(0.168)$ & $0.055(0.009)$ \\
\hline $2007 b c$ & CFA3 & $0.022(0.001)$ & $54200.56(0.20)$ & 0.1130 & $-1.315(0.107)$ & $0.016(0.030)$ & $15.660(0.035)$ & $34.781(0.230)$ & $0.018(0.003)$ \\
\hline 2007bd & CFA3 & $0.032(0.001)$ & $54206.64(0.10)$ & 1.0000 & $-1.356(0.104)$ & $-0.048(0.030)$ & $16.315(0.036)$ & $35.635(0.212)$ & $0.027(0.004)$ \\
\hline $2007 \mathrm{ca}$ & CFA3 & $0.015(0.001)$ & $54227.85(0.12)$ & 0.3870 & $0.553(0.115)$ & $0.217(0.030)$ & $15.691(0.049)$ & $34.429(0.256)$ & $0.054(0.009)$ \\
\hline 2007ci & CFA3 & $0.019(0.001)$ & $54246.73(0.10)$ & 0.6890 & $-2.753(0.188)$ & $0.030(0.035)$ & $15.615(0.042)$ & $34.488(0.172)$ & $0.021(0.003)$ \\
\hline 2007co & CFA3 & $0.027(0.001)$ & $54265.13(0.11)$ & 1.0000 & $-0.162(0.095)$ & $0.079(0.030)$ & $16.266(0.063)$ & $35.347(0.149)$ & $0.081(0.013)$ \\
\hline $2007 \mathrm{cp}$ & CFA3 & $0.038(0.001)$ & $54269.75(0.45)$ & 0.0345 & $-2.905(1.078)$ & $-0.026(0.059)$ & $16.905(0.062)$ & $35.936(0.276)$ & $0.036(0.006)$ \\
\hline $2007 \mathrm{cq}$ & CFA3 & $0.025(0.001)$ & $54281.04(0.12)$ & 0.5920 & $-0.642(0.163)$ & $-0.037(0.030)$ & $15.605(0.062)$ & $34.988(0.152)$ & $0.079(0.013)$ \\
\hline 2007qe & CFA3 & $0.024(0.001)$ & $54429.80(0.06)$ & 0.9870 & $0.689(0.076)$ & $0.048(0.027)$ & $15.803(0.036)$ & $35.102(0.155)$ & $0.031(0.005)$ \\
\hline
\end{tabular}


Table 7

(Continued)

\begin{tabular}{|c|c|c|c|c|c|c|c|c|c|}
\hline $\mathrm{SN}$ & Set & $z_{\mathrm{cmb}}$ & $t_{\text {peak }}$ & $P_{\text {fit }}$ & $x_{1}$ & $c$ & $m_{B}$ & $\mu$ & $E(B-V)$ \\
\hline $2008 \mathrm{~L}$ & CFA3 & $0.019(0.001)$ & $54493.39(0.42)$ & 0.7630 & $-1.550(0.155)$ & $-0.089(0.042)$ & $14.977(0.091)$ & $34.399(0.180)$ & $0.118(0.019)$ \\
\hline 2008bf & CFA3 & $0.022(0.001)$ & $54555.01(0.15)$ & 9610 & $0.319(0.103)$ & $-0.023(0.028)$ & $15.464(0.035)$ & $34.939(0.158)$ & $.027(0.004)$ \\
\hline 2007A & CFA4 & $0.017(0.001)$ & $54113.33(0.21)$ & 0.9540 & $0.427(0.272)$ & $0.068(0.037)$ & $15.379(0.059)$ & $34.575(0.314)$ & $0.059(0.010)$ \\
\hline $2007 \mathrm{fb}$ & CFA4 & $0.017(0.001)$ & $54288.69(0.87)$ & 0.9940 & $-1.748(0.474)$ & $-0.118(0.047)$ & $15.267(0.069)$ & $34.754(0.243)$ & $0.045(0.007)$ \\
\hline 2007hu & CFA4 & $0.036(0.001)$ & $54353.23(2.11)$ & 0.5630 & $-1.257(0.526)$ & $0.210(0.076)$ & $17.458(0.153)$ & $35.965(0.262)$ & $0.037(0.006)$ \\
\hline 2007kh & CFA4 & $0.050(0.001)$ & $54365.04(0.15)$ & 0.6680 & $0.978(0.977)$ & $0.087(0.112)$ & $17.536(0.264)$ & $36.748(0.482)$ & $0.147(0.023)$ \\
\hline 2007jg & CFA4 & $0.037(0.001)$ & $54366.42(0.52)$ & 0.9570 & $-0.714(0.154)$ & $-0.056(0.039)$ & $16.968(0.071)$ & $36.403(0.280)$ & $0.079(0.013)$ \\
\hline 2007is & CFA4 & $0.030(0.001)$ & $54366.93(0.73)$ & 0.6780 & $-0.462(0.213)$ & $0.031(0.037)$ & $16.198(0.046)$ & $35.388(0.207)$ & $0.016(0.003)$ \\
\hline $2007 \mathrm{kk}$ & CFA4 & $0.041(0.001)$ & $54383.80(0.38)$ & 1.0000 & $0.847(0.172)$ & $-0.022(0.041)$ & $16.710(0.113)$ & $36.255(0.194)$ & $0.169(0.027)$ \\
\hline 2007nq & CFA4 & $0.044(0.001)$ & $54398.31(0.56)$ & 1.0000 & $-1.599(0.198)$ & $-0.066(0.038)$ & $17.118(0.058)$ & $36.462(0.280)$ & $0.029(0.005)$ \\
\hline $2007 \mathrm{su}$ & CFA4 & $0.027(0.001)$ & $54460.46(0.89)$ & 0.9990 & $0.311(0.387)$ & $0.172(0.041)$ & $16.634(0.078)$ & $35.481(0.208)$ & $0.067(0.011)$ \\
\hline 2007ux & CFA4 & $0.032(0.001)$ & $54465.77(0.52)$ & 0.4970 & $-2.420(0.138)$ & $0.154(0.045)$ & $17.136(0.068)$ & $35.660(0.214)$ & $0.036(0.006)$ \\
\hline $2008 \mathrm{C}$ & CFA4 & $0.017(0.001)$ & $54467.32(0.45)$ & 1.0000 & $-0.694(0.167)$ & $0.096(0.035)$ & $15.339(0.067)$ & $34.290(0.217)$ & $0.068(0.011)$ \\
\hline 2007sw & CFA4 & $0.026(0.001)$ & $54468.52(0.34)$ & 0.1920 & $0.121(0.144)$ & $0.098(0.033)$ & $16.035(0.041)$ & $35.095(0.201)$ & $0.015(0.002)$ \\
\hline $2008 Y$ & CFA4 & $0.070(0.001)$ & $54503.69(1.02)$ & 0.6160 & $-0.565(0.277)$ & $0.065(0.042)$ & $18.260(0.064)$ & $37.327(0.204)$ & $0.010(0.002)$ \\
\hline $2008 Z$ & CFA4 & $0.022(0.001)$ & $54515.48(0.09)$ & 0.5980 & $1.010(0.128)$ & $0.081(0.028)$ & $16.159(0.033)$ & $35.398(0.202)$ & $0.009(0.001)$ \\
\hline 2008at & CFA4 & $0.035(0.001)$ & $54526.39(1.28)$ & 0.9710 & $-1.501(0.249)$ & $0.212(0.064)$ & $17.319(0.109)$ & $35.785(0.263)$ & $0.074(0.012)$ \\
\hline 2008ar & CFA4 & $0.027(0.001)$ & $54534.69(0.11)$ & 1.0000 & $0.003(0.096)$ & $-0.047(0.028)$ & $15.978(0.036)$ & $35.484(0.196)$ & $0.030(0.005)$ \\
\hline $2008 \mathrm{bz}$ & CFA4 & $0.061(0.001)$ & $54578.11(0.93)$ & 0.8500 & $-0.119(0.365)$ & $-0.129(0.040)$ & $17.641(0.059)$ & 37.393(0.204) & $0.025(0.004)$ \\
\hline $2008 \mathrm{cf}$ & CFA4 & $0.047(0.001)$ & $54594.73(1.11)$ & 1.0000 & $1.475(0.570)$ & $-0.135(0.038)$ & $16.728(0.054)$ & $36.720(0.217)$ & $0.054(0.009)$ \\
\hline 2008051 & CFA4 & $0.040(0.001)$ & $54616.65(0.46)$ & 0.6820 & $0.823(0.172)$ & $-0.135(0.032)$ & $16.364(0.045)$ & $36.265(0.195)$ & $0.035(0.006)$ \\
\hline 2008050 & CFA4 & $0.049(0.001)$ & $54621.12(0.99)$ & 0.7890 & $1.038(0.556)$ & $0.024(0.0$ & $16.901(0.046)$ & $36.325(0.215)$ & $0.021(0.003)$ \\
\hline $2008 \mathrm{fr}$ & CFA4 & $0.048(0.001)$ & $54732.41(0.55)$ & 1.0000 & $0.733(0.155)$ & $-0.135(0.032)$ & $16.525(0.046)$ & $36.416(0.193)$ & $0.036(0.006)$ \\
\hline $2008 \mathrm{gb}$ & CFA4 & $0.036(0.001)$ & $54747.06(0.71)$ & 1.0000 & $0.067(0.299)$ & $-0.016(0.042)$ & $16.823(0.103)$ & $36.239(0.204)$ & $0.147(0.023)$ \\
\hline $2008 \mathrm{gl}$ & CFA4 & $0.033(0.001)$ & $54768.47(0.16)$ & 1.0000 & $-1.348(0.135)$ & $-0.032(0.032)$ & 16.541( & 35.809 & $0.004)$ \\
\hline 2008hj & CFA4 & $0.037(0.001)$ & $54803.84(0.79)$ & 0.0250 & $-0.536(0.376)$ & $-0.104(0.0$ & 16.505 & $36.116(0.2$ & $0.029(0.005)$ \\
\hline $2008 \mathrm{hm}$ & CFA4 & $0.019(0.001)$ & $54805.18(0.21)$ & 0.9370 & $0.175(0.127)$ & $-0.003(0.0$ & $15.461(0.2$ & 3485000 & $0.327(0.052)$ \\
\hline 2008hv & CFA4 & $0.014(0.001)$ & $54817.10(0.11)$ & 1.0000 & $-1.401(0.129)$ & $-0.122(0.032)$ & $14.439(0.041)$ & & $0.026(0.004)$ \\
\hline 2009D & CFA4 & $0.025(0.001)$ & $54842.06(0.43)$ & 0.9990 & $0.419(0.150)$ & $-0.054(0.032)$ & $15.474(0.049)$ & $35.059(0.201)$ & $0.043(0.007)$ \\
\hline 2009Y & CFA4 & $0.010(0.001)$ & $54876.23(0.27)$ & 0.9000 & $0.376(0.101)$ & $0.034(0.029)$ & $13.677(0.059)$ & $32.976(0.261)$ & $0.075(0.012)$ \\
\hline 2009ad & CFA4 & $0.029(0.001)$ & $54886.60(0.18)$ & 1.0000 & $0.180(0.106)$ & $-0.062(0.032)$ & $15.928(0.065)$ & $35.508(0.196)$ & $0.083(0.013)$ \\
\hline 2009al & CFA4 & $0.024(0.001)$ & $54897.31(0.14)$ & 0.0082 & $-0.447(0.121)$ & $0.069(0.029)$ & $15.951(0$. & $35.025(0.202)$ & $0.020(0.003)$ \\
\hline $2009 \mathrm{ds}$ & CFA4 & $0.021(0.001)$ & $54961.10(0.31)$ & 0.9580 & $0.498(0.166)$ & $-0.052(0.034)$ & $15.227(0.045)$ & $34.820(0.211)$ & $0.031(0.005)$ \\
\hline 2009kk & CFA4 & $0.013(0.001)$ & $55126.85(0.37)$ & 1.0000 & $-1.216(0.113)$ & $-0.080(0.037)$ & $14.446(0.082)$ & $33.886(0.240)$ & $0.118(0.019)$ \\
\hline 2009lf & CFA4 & $0.044(0.001)$ & $55150.41(0.48)$ & 0.3280 & $-1.677(0.110)$ & $-0.040(0.037)$ & $16.672(0.054)$ & $35.920(0.214)$ & $0.045(0.007)$ \\
\hline $2009 \mathrm{kq}$ & CFA4 & $0.013(0.001)$ & $55155.14(0.29)$ & 1.0000 & $-0.040(0.176)$ & $-0.013(0.034)$ & $14.249(0.046)$ & $33.641(0.239)$ & $0.035(0.006)$ \\
\hline 2009le & CFA4 & $0.017(0.001)$ & $55166.08(0.10)$ & 0.9850 & $-0.000(0.207)$ & $0.037(0$ & 15.041 & 34.277 & 0.01 \\
\hline 2009na & CFA4 & $0.022(0.001)$ & $55201.98(0.14)$ & 0.8080 & $-0.738(0.137)$ & $-0.011(0$ & $15.543(0$ & 34.830 & $0.027(0.004)$ \\
\hline 2009nq & CFA4 & $0.015(0.001)$ & $55203.73(0.86)$ & 1.0000 & $-0.243(0.830)$ & $0.049(0.0$ & 15 & 5) & $0.125(0.020)$ \\
\hline 2010A & CFA4 & $0.020(0.001)$ & $55212.45(0.16)$ & 0.9460 & $0.461(0.169)$ & $0.116(0.037)$ & $15.589(0.043)$ & $34.639(0.219)$ & $0.025(0.004)$ \\
\hline 2010Y & CFA4 & $0.011(0.001)$ & $55247.93(0.13)$ & 0.9900 & $-2.430(0.139)$ & $-0.040(0.036)$ & $14.743(0.042)$ & $33.885(0.250)$ & $0.012(0.002)$ \\
\hline 2010ag & CFA4 & $0.034(0.001)$ & $55269.46(0.68)$ & 0.0109 & $1.332(0.256)$ & $0.067(0.034)$ & $16.427(0.043)$ & $35.754(0.204)$ & $0.027(0.004)$ \\
\hline 2010ai & CFA4 & $0.019(0.001)$ & $55277.23(0.09)$ & 1.0000 & $-1.563(0.130)$ & $-0.075(0.034)$ & $15.772(0.039)$ & $35.147(0.210)$ & $0.008(0.001)$ \\
\hline 2010dw & CFA4 & $0.039(0.001)$ & $55358.30(0.39)$ & 0.3610 & $0.317(0.182)$ & $0.089(0.034)$ & $16.900(0.061)$ & $36.014(0.197)$ & $0.080(0.013)$ \\
\hline $2010 \mathrm{dt}$ & CFA4 & $0.053(0.001)$ & $55361.66(0.25)$ & 0.2690 & $-0.007(0.607)$ & $-0.071(0.054)$ & $17.379(0.079)$ & $36.962(0.207)$ & $0.029(0.005)$ \\
\hline $2004 \mathrm{ef}$ & CSP & $0.030(0.001)$ & $53264.17(0.05)$ & 1.0000 & $-1.274(0.043)$ & $0.076(0.021)$ & $16.595(0.036)$ & $35.530(0.189)$ & $0.044(0.007)$ \\
\hline 2004 eo & CSP & $0.015(0.001)$ & $53278.34(0.04)$ & 0.9970 & $-1.151(0.040)$ & $0.002(0.024)$ & $14.791(0.059)$ & $33.979(0.171)$ & $0.081(0.013)$ \\
\hline 2004ey & CSP & $0.016(0.001)$ & $53304.48(0.06)$ & 1.0000 & $0.051(0.042)$ & $-0.103(0.025)$ & $14.468(0.071)$ & $34.159(0.170)$ & $0.103(0.016)$ \\
\hline $2004 \mathrm{gs}$ & CSP & $0.027(0.001)$ & $53355.60(0.14)$ & 0.5630 & $-1.682(0.056)$ & $0.126(0.022)$ & $16.886(0.027)$ & $35.603(0.138)$ & $0.025(0.004)$ \\
\hline 2004gu & CSP & $0.047(0.001)$ & $53362.46(0.23)$ & 1.0000 & $1.320(0.116)$ & $0.059(0.021)$ & $17.162(0.025)$ & $36.512(0.128)$ & $0.021(0.003)$ \\
\hline $2005 \mathrm{M}$ & CSP & $0.023(0.001)$ & $53406.15(0.06)$ & 1.0000 & $1.170(0.063)$ & $-0.021(0.020)$ & $15.645(0.025)$ & $35.233(0.202)$ & $0.025(0.004)$ \\
\hline 2005ag & CSP & $0.080(0.001)$ & $53415.11(0.17)$ & 1.0000 & $0.134(0.080)$ & $-0.043(0.025)$ & $18.201(0.030)$ & $37.713(0.127)$ & $0.033(0.005)$ \\
\hline 2005al & CSP & $0.012(0.001)$ & $53430.65(0.18)$ & 1.0000 & $-1.183(0.056)$ & $-0.090(0.022)$ & $14.611(0.037)$ & $34.089(0.193)$ & $0.044(0.007)$ \\
\hline $2005 \mathrm{bg}$ & CSP & $0.025(0.001)$ & $53470.15(0.27)$ & 0.7460 & $0.288(0.120)$ & $-0.051(0.021)$ & $15.595(0.028)$ & $35.155(0.141)$ & $0.026(0.004)$ \\
\hline 2005bo & CSP & $0.014(0.001)$ & $53478.56(0.30)$ & 1.0000 & $-0.940(0.169)$ & $0.200(0.023)$ & $15.372(0.036)$ & $33.956(0.179)$ & $0.037(0.006)$ \\
\hline $2005 \mathrm{el}$ & CSP & $0.015(0.001)$ & $53646.64(0.09)$ & 0.9960 & $-1.212(0.077)$ & $-0.141(0.025)$ & $14.589(0.062)$ & $34.224(0.245)$ & $0.084(0.013)$ \\
\hline 2005 eq & CSP & $0.029(0.001)$ & $53654.93(0.21)$ & 1.0000 & $1.232(0.107)$ & $-0.023(0.023)$ & $16.002(0.046)$ & $35.602(0.194)$ & $0.060(0.010)$ \\
\hline $2005 \mathrm{hc}$ & CSP & $0.045(0.001)$ & $53667.75(0.13)$ & 1.0000 & $0.691(0.088)$ & $-0.023(0.021)$ & $17.046(0.025)$ & $36.570(0.179)$ & $0.024(0.004)$ \\
\hline 2005hj & CSP & $0.057(0.001)$ & $53674.46(0.38)$ & 0.3750 & $1.419(0.187)$ & $-0.029(0.023)$ & $17.466(0.027)$ & 37.114(0.186) & $0.025(0.004)$ \\
\hline 2005ir & CSP & $0.075(0.001)$ & $53685.50(0.22)$ & 0.6950 & $0.399(0.170)$ & $-0.005(0.022)$ & $18.171(0.026)$ & $37.598(0.177)$ & $0.025(0.004)$ \\
\hline 2005 iq & CSP & $0.034(0.001)$ & $53687.65(0.09)$ & 1.0000 & $-1.001(0.074)$ & $-0.108(0.021)$ & $16.498(0.023)$ & $36.058(0.186)$ & $0.018(0.003)$ \\
\hline $2005 \mathrm{kc}$ & CSP & $0.015(0.001)$ & $53697.70(0.06)$ & 0.9990 & $-0.696(0.072)$ & $0.148(0.026)$ & $15.268(0.070)$ & $34.053(0.247)$ & $0.098(0.016)$ \\
\hline 2005ki & CSP & $0.020(0.001)$ & $53705.32(0.09)$ & 0.9300 & $-1.349(0.056)$ & $-0.101(0.022)$ & $15.275(0.028)$ & $34.765(0.214)$ & $0.026(0.004)$ \\
\hline $2005 \mathrm{mc}$ & CSP & $0.026(0.001)$ & $53731.24(0.34)$ & 0.9020 & $-1.802(0.073)$ & $0.192(0.025)$ & $16.766(0.043)$ & $35.256(0.203)$ & $0.036(0.006)$ \\
\hline 2005na & CSP & $0.027(0.001)$ & $53741.13(0.14)$ & 0.2010 & $-0.584(0.062)$ & $-0.096(0.023)$ & $15.684(0.048)$ & $35.265(0.197)$ & $0.063(0.010)$ \\
\hline 2006ax & CSP & $0.017(0.001)$ & $53827.53(0.04)$ & 1.0000 & $0.122(0.049)$ & $-0.097(0.020)$ & $14.744(0.034)$ & $34.425(0.225)$ & $0.040(0.006)$ \\
\hline
\end{tabular}


Table 7

(Continued)

\begin{tabular}{|c|c|c|c|c|c|c|c|c|c|}
\hline SN & Set & $z_{\mathrm{cmb}}$ & $t_{\text {peak }}$ & $P_{\text {fit }}$ & $x_{1}$ & $c$ & $m_{B}$ & $\mu$ & $E(B-V)$ \\
\hline 2006bh & CSP & $0.011(0.001)$ & $53833.42(0.10)$ & 1.0000 & $-1.545(0.058)$ & $-0.064(0.021)$ & $14.089(0.025)$ & $33.431(0.207)$ & $0.021(0.003)$ \\
\hline 2006еj & CSP & $0.020(0.001)$ & $53976.06(0.32)$ & 1.0000 & $-1.421(0.081)$ & $-0.030(0.024)$ & $15.438(0.035)$ & $34.689(0.154)$ & $0.028(0.005)$ \\
\hline $2006 \mathrm{ev}$ & CSP & $0.028(0.001)$ & $53989.44(0.31)$ & 1.0000 & $-1.261(0.079)$ & $0.097(0.027)$ & $16.811(0.060)$ & $35.681(0.140)$ & $0.073(0.012)$ \\
\hline 2006et & CSP & $0.022(0.001)$ & $53994.20(0.07)$ & 1.0000 & $0.676(0.070)$ & $0.130(0.020)$ & $15.702(0.023)$ & $34.737(0.205)$ & $0.015(0.002)$ \\
\hline $2006 \mathrm{kf}$ & CSP & $0.021(0.001)$ & $54040.91(0.14)$ & 0.9980 & $-1.827(0.090)$ & $-0.049(0.037)$ & $15.662(0.120)$ & $34.918(0.225)$ & $0.182(0.029)$ \\
\hline $2006 \mathrm{ob}$ & CSP & $0.059(0.001)$ & $54063.10(0.25)$ & 0.9990 & $-1.884(0.145)$ & $-0.052(0.027)$ & $17.930(0.032)$ & $37.189(0.192)$ & $0.027(0.004)$ \\
\hline 2006ot & CSP & $0.052(0.001)$ & $54063.66(0.47)$ & 0.0458 & $0.695(0.138)$ & $0.101(0.023)$ & $17.703(0.031)$ & $36.832(0.129)$ & $0.018(0.003)$ \\
\hline 2006py & CSP & $0.057(0.001)$ & $54070.95(0.44)$ & 0.9740 & $0.076(0.348)$ & $-0.001(0.025)$ & $17.608(0.042)$ & $36.976(0.132)$ & $0.053(0.008)$ \\
\hline $2007 \mathrm{~A}$ & CSP & $0.017(0.001)$ & $54113.18(0.16)$ & 0.9920 & $0.561(0.214)$ & $0.090(0.025)$ & $15.397(0.048)$ & $34.541(0.236)$ & $0.059(0.010)$ \\
\hline 2007ai & CSP & $0.032(0.001)$ & $54173.80(0.37)$ & 1.0000 & $0.976(0.156)$ & $0.156(0.044)$ & $16.901(0.143)$ & $35.895(0.216)$ & $0.215(0.034)$ \\
\hline 2007as & CSP & $0.018(0.001)$ & $54181.66(0.22)$ & 0.0177 & $-0.890(0.082)$ & $-0.094(0.027)$ & $15.183(0.082)$ & $34.714(0.153)$ & $0.124(0.020)$ \\
\hline $2007 b c$ & CSP & $0.022(0.001)$ & $54200.46(0.22)$ & 1.0000 & $-1.109(0.092)$ & $0.036(0.023)$ & $15.651(0.025)$ & $34.736(0.212)$ & $0.018(0.003)$ \\
\hline 2007bd & CSP & $0.032(0.001)$ & $54206.84(0.07)$ & 0.8540 & $-0.953(0.072)$ & $-0.067(0.022)$ & $16.273(0.029)$ & $35.708(0.190)$ & $0.027(0.004)$ \\
\hline $2007 \mathrm{ca}$ & CSP & $0.015(0.001)$ & $54227.80(0.08)$ & 0.9970 & $0.573(0.076)$ & $0.200(0.023)$ & $15.628(0.042)$ & $34.422(0.242)$ & $0.053(0.008)$ \\
\hline $2007 h x$ & CSP & $0.079(0.001)$ & $54354.56(0.43)$ & 0.6350 & $0.139(0.174)$ & $0.116(0.025)$ & $19.018(0.029)$ & $38.022(0.131)$ & $0.022(0.004)$ \\
\hline 2007jh & CSP & $0.041(0.001)$ & $54366.71(0.16)$ & 0.4850 & $-0.517(0.098)$ & $-0.018(0.028)$ & $17.031(0.057)$ & $36.370(0.133)$ & $0.077(0.012)$ \\
\hline $2007 \mathrm{jg}$ & CSP & $0.037(0.001)$ & $54366.77(0.16)$ & 0.1420 & $-0.616(0.098)$ & $0.043(0.023)$ & $17.086(0.055)$ & $36.219(0.182)$ & $0.079(0.013)$ \\
\hline $2007 \mathrm{nq}$ & CSP & $0.044(0.001)$ & $54398.17(0.25)$ & 1.0000 & $-1.784(0.090)$ & $-0.019(0.024)$ & $17.149(0.030)$ & $36.316(0.185)$ & $0.022(0.004)$ \\
\hline 2008R & CSP & $0.013(0.001)$ & $54493.00(0.26)$ & 0.4100 & $-2.000(0.084)$ & $0.036(0.025)$ & $14.968(0.045)$ & $33.927(0.190)$ & $0.053(0.008)$ \\
\hline $2008 b c$ & CSP & $0.016(0.001)$ & $54550.14(0.05)$ & 0.9220 & $0.430(0.058)$ & $-0.146(0.044)$ & $14.178(0.166)$ & $34.060(0.163)$ & $0.292(0.047)$ \\
\hline $2008 \mathrm{bq}$ & CSP & $0.034(0.001)$ & $54563.89(0.22)$ & 1.0000 & $0.245(0.082)$ & $0.061(0.024)$ & $16.479(0.055)$ & $35.674(0.131)$ & $0.075(0.012)$ \\
\hline 2008gp & CSP & $0.033(0.001)$ & $54779.20(0.06)$ & 0.9990 & $-0.178(0.054)$ & $-0.073(0.025)$ & $16.175(0.063)$ & $35.741(0.132)$ & $0.090(0.014)$ \\
\hline $2008 \mathrm{ia}$ & CSP & $0.023(0.001)$ & $54813.07(0.14)$ & 0.9840 & $-1.308(0.081)$ & $-0.029(0.032)$ & $15.702(0.103)$ & $34.967(0.146)$ & $0.159(0.025)$ \\
\hline $2008 \mathrm{hv}$ & CSP & $0.014(0.001)$ & $54817.02(0.06)$ & 0.9910 & $-1.260(0.064)$ & $-0.072(0.021)$ & $14.460(0.028)$ & $33.870(0.254)$ & $0.026(0.004)$ \\
\hline
\end{tabular}

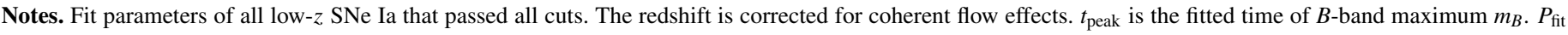

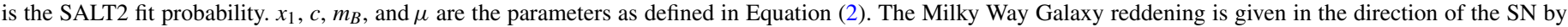
Schlegel et al. (1998), and corrected by $6 \%-14 \%$ as described in S14.

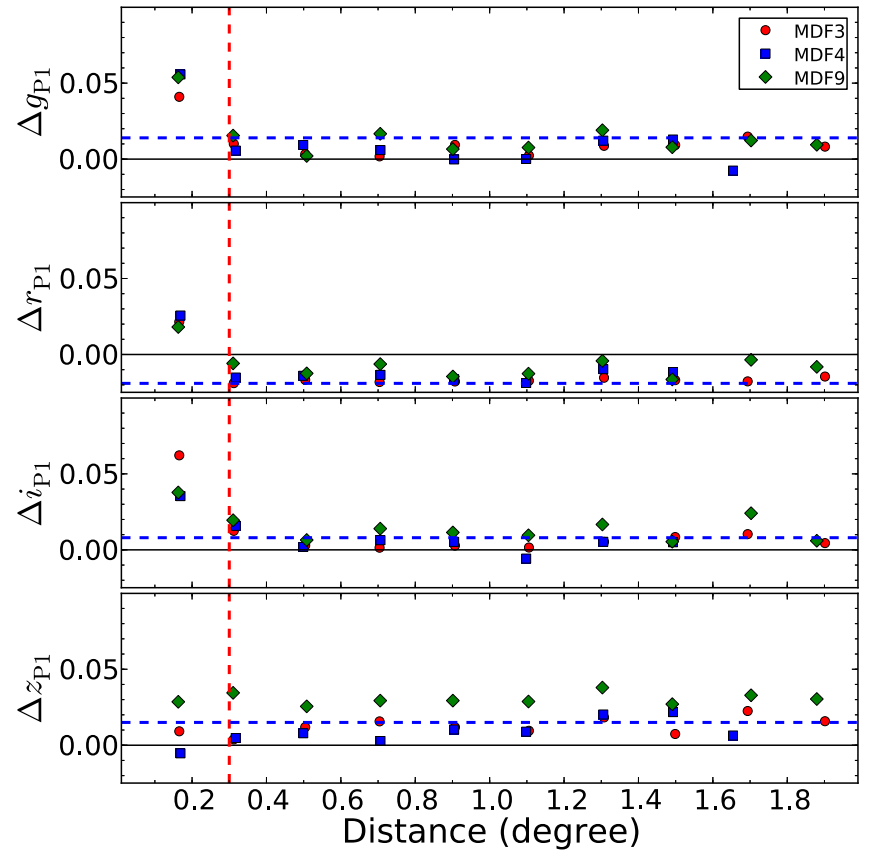

Figure 10. Median differences between the PS1 photometry and the synthetic PS1 photometry derived from SDSS using Equation (6) and Table 6 from $\mathrm{T} 12 \mathrm{~b}$ using stars in the color range $0.5<(g-r)_{\mathrm{SDSS}}<0.8$ for $g r i_{\mathrm{P} 1}$ and $0.8<(r-i)_{\text {SDSS }}<1.1$ for $z_{\mathrm{P} 1}$. The median is calculated in bins of distance between the stars and the center of the MDFs. We exclude all SNe that are within $0.3 \mathrm{deg}$ of the field center (red dashed lines), since in the photometric calibration in the field center is unreliable due to strong spatial variation in the PSF. The blue lines indicate the known offsets between SDSS DR8 and the Pan-STARRS1 calibration by T12b, as shown in Table 1 of S14.

(A color version of this figure is available in the online journal.) across the focal plane are $<3 \mathrm{mmag}$ for stars with colors around $0.4<g-i<1.4$. The photometry of SNe has a stronger dependence on these spatial variations in the filter passbands due to the broad absorption features in their spectra. As described in $\mathrm{S} 14$, we fit each SN light curve using the expected filter function at the position of the $\mathrm{SN}$ on the focal plane. Accounting for the variation of the filter functions may change the distances from their nominal values by $\sim 3 \mathrm{mmag}$. These differences are shown in S14 (Figure 2) and are included in the distances presented in our Table 8.

Over time, the optical components may degrade or change. Color-dependent changes could introduce a systematic bias into the photometry. In Figure 11, we show the median variation $\Delta g_{\mathrm{P} 1}$ in the average magnitude of stars at various epochs for different colors $\left(g_{\mathrm{P} 1}-r_{\mathrm{P} 1}\right)$. Similar results are seen in each band. Typically, the median is not different than zero to within $3 \mathrm{mmag}$, and the standard deviation in a given bin is on the order of 5 mmag. No long-term trends can be discerned, and we set the upper limit on any long-term changes of the optical system to 3 mmag.

\section{LIGHT CURVE FITS AND SAMPLE CUTS}

\subsection{SALT2 Light Curve Fits}

Many light curve fitters have been developed over the last decade (e.g., MLCS2K2, Jha et al. 2007; SALT, Guy et al. 2007; SiFTO, Conley et al. (2008); and BayeSN, Mandel et al. $2009,2011)$. Each method makes corrections for the light curve shape and observed color of the SN. However, there are two fundamentally different ways to apply the color correction. The first empirically determines the correlation between observed 
Table 8

Fit Parameters of the Pan-STARRS1 Spectroscopic Sample

\begin{tabular}{|c|c|c|c|c|c|c|c|c|c|}
\hline PS1 Name & $z_{\mathrm{cmb}}$ & $t_{\text {peak }}$ & $P_{\text {fit }}$ & $x_{1}$ & $c$ & $m_{B}$ & $\mu$ & $E(B-V)$ & $\begin{array}{l}\text { Host } \\
\text { Mass }\end{array}$ \\
\hline PS1-0909006 & $0.283(0.001)$ & $55075.41(2.59)$ & 0.745 & $-0.726(1.428)$ & $0.008(0.073)$ & $21.352(0.094)$ & $40.579(0.309)$ & $0.035(0.006)$ & 9.1620 \\
\hline PS1-0910017 & $0.319(0.005)$ & $55109.65(0.57)$ & .902 & $-0.704(0.473)$ & $-0.115(0.037)$ & $21.412(0.045)$ & $41.035(0.129)$ & $0.018(0.003)$ & $\cdots$ \\
\hline PS1-0910018 & $0.264(0.001)$ & $55109.23(0.59)$ & 0.246 & $-2.253(0.625)$ & $-0.174(0.050)$ & $20.975(0.055)$ & $40.573(0.135)$ & $0.020(0.003)$ & $\ldots$ \\
\hline PS1-10c & $0.153(0.001)$ & $55210.25(0.22)$ & 0.930 & $-2.147(0.169)$ & $0.020(0.033)$ & $20.284(0.038)$ & $39.276(0.114)$ & $0.022(0.003)$ & 8.3920 \\
\hline PS1-10d & $0.230(0.001)$ & $55204.10(0.51)$ & 0.742 & $-0.222(0.482)$ & $0.124(0.041)$ & $21.408(0.049)$ & $40.336(0.133)$ & $0.007(0.001)$ & 10.5900 \\
\hline PS1-10e & $0.246(0.001)$ & $55215.72(0.30)$ & 0.084 & $0.730(0.274)$ & $0.020(0.028)$ & $21.164(0.037)$ & $40.557(0.097)$ & $0.018(0.003)$ & 11.0200 \\
\hline PS1-10g & $0.136(0.001)$ & $55201.05(0.63)$ & 0.992 & $0.634(0.744)$ & $-0.077(0.040)$ & $19.394(0.052)$ & $39.085(0.135)$ & $0.021(0.003)$ & 10.4100 \\
\hline PS1-10h & $0.249(0.005)$ & $55207.44(0.42)$ & 0.720 & $-0.099(0.499)$ & $-0.107(0.032)$ & $20.710(0.040)$ & $40.392(0.127)$ & $0.022(0.004)$ & $\cdots$ \\
\hline PS1-10i & $0.149(0.001)$ & $55203.68(0.26)$ & 0.601 & $-0.121(0.291)$ & $-0.058(0.036)$ & $19.614(0.044)$ & $39.137(0.113)$ & $0.023(0.004)$ & $\cdots$ \\
\hline PS $1-10 \mathrm{j}$ & $0.436(0.001)$ & $55213.59(0.60)$ & 0.931 & $-0.510(0.579)$ & $-0.001(0.046)$ & $22.853(0.047)$ & $42.141(0.165)$ & $0.007(0.001)$ & 11.3200 \\
\hline PS1-10k & $0.341(0.001)$ & $55216.36(0.27)$ & 0.965 & $0.273(0.255)$ & $-0.030(0.027)$ & $21.797(0.032)$ & $41.286(0.122)$ & $0.015(0.002)$ & 9.6370 \\
\hline PS1-101 & $0.371(0.001)$ & $55214.50(0.39)$ & 0.997 & $1.335(0.308)$ & $-0.083(0.028)$ & $21.793(0.033)$ & $41.602(0.105)$ & $0.024(0.004)$ & 8.5220 \\
\hline PS1-10m & $0.619(0.001)$ & $55215.12(0.81)$ & 0.650 & $1.536(0.706)$ & $-0.166(0.050)$ & $23.197(0.048)$ & $43.300(0.197)$ & $0.015(0.002)$ & 9.0170 \\
\hline PS1-10n & $0.461(0.001)$ & $55222.82(0.39)$ & 0.792 & $1.196(0.397)$ & $-0.103(0.033)$ & $22.477(0.039)$ & $42.328(0.133)$ & $0.016(0.003)$ & 8.8120 \\
\hline PS1-10o & $0.221(0.001)$ & $55227.64(0.15)$ & 0.119 & $-0.771(0.191)$ & $-0.114(0.030)$ & $20.605(0.036)$ & $40.218(0.103)$ & $0.016(0.003)$ & 11.3100 \\
\hline PS1-10p & $0.311(0.001)$ & $55223.21(0.20)$ & 0.899 & $-1.124(0.242)$ & $-0.115(0.034)$ & $21.611(0.039)$ & $41.178(0.119)$ & $0.017(0.003)$ & 9.1670 \\
\hline PS1-10r & $0.119(0.001)$ & $55213.12(0.18)$ & 0.808 & $-0.122(0.207)$ & $0.111(0.035)$ & $19.634(0.040)$ & $38.617(0.112)$ & $0.006(0.001)$ & 11.0000 \\
\hline PS1-10v & $0.100(0.001)$ & $55238.92(0.30)$ & 0.992 & $1.035(0.286)$ & $0.039(0.032)$ & $19.132(0.041)$ & $38.507(0.110)$ & $0.019(0.003)$ & 9.9770 \\
\hline PS1-10w & $0.032(0.001)$ & $55248.01(0.11)$ & 0.775 & $-0.504(0.128)$ & $-0.005(0.038)$ & $16.436(0.045)$ & $35.736(0.130)$ & $0.006(0.001)$ & 10.2000 \\
\hline PS1-10z & $0.141(0.001)$ & $55235.96(0.30)$ & 0.898 & $-2.375(0.396)$ & $0.045(0.043)$ & $20.303(0.052)$ & $39.180(0.127)$ & $0.024(0.004)$ & 7.4570 \\
\hline PS1-10af & $0.244(0.001)$ & $55216.97(0.24)$ & 0.756 & $-0.466(0.279)$ & $0.073(0.033)$ & $21.417(0.040)$ & $40.472(0.111)$ & $0.006(0.001)$ & 10.4800 \\
\hline PS1-10aj & $0.196(0.001)$ & $55210.94(0.20)$ & 0.399 & $-0.220(0.166)$ & $-0.045(0.027)$ & $20.337(0.035)$ & $39.807(0.099)$ & $0.025(0.004)$ & 10.7100 \\
\hline PS1-10hu & $0.127(0.001)$ & $55270.80(0.64)$ & 0.947 & $0.155(0.298)$ & $0.062(0.037)$ & $19.372(0.040)$ & $38.551(0.136)$ & $0.011(0.002)$ & $\cdots$ \\
\hline PS1-10ia & $0.409(0.001)$ & $55272.94(1.28)$ & 0.006 & $-0.434(1.223)$ & $0.028(0.068)$ & $22.395(0.072)$ & $41.599(0.307)$ & 0.010 & 11.5900 \\
\hline PS1-10if & $0.151(0.001)$ & $55277.31(0.52)$ & 0.283 & $-1.987(0.565)$ & $0.127(0.061)$ & $20.730(0.062)$ & $39.399(0.224)$ & $0.016(0.003)$ & 10.6300 \\
\hline PS1-10ig & $0.260(0.001)$ & $55270.32(0.65)$ & 0.996 & $0.771(0.502)$ & $-0.077(0.033)$ & $20.946(0.038)$ & $40.655(0.149)$ & $0.007(0.001)$ & 8.4220 \\
\hline PS1-10ik & $0.231(0.001)$ & $55281.52(0.37)$ & 0.988 & $-0.442(0.324)$ & $-0.113(0.044)$ & $20.664(0.046)$ & $40.318(0.153)$ & 0.0 & 8.9120 \\
\hline PS1-10im & $0.510(0.001)$ & $55288.90(1.03)$ & 0.911 & $0.391(1.235)$ & $-0.114(0.082)$ & $22.503(0.145)$ & $42.277(0.275)$ & 0.00 & 9.5470 \\
\hline PS1-10io & $0.221(0.001)$ & $55300.14(0.65)$ & 0.888 & $1.358(0.482)$ & $-0.126(0.043)$ & & $40.331(0.128)$ & & 8.9120 \\
\hline PS1-10ir & $0.251(0.001)$ & $55337.37(0.25)$ & 0.987 & $-0.126(0.379)$ & $-0.034(0.036)$ & $21.147(0.050)$ & $40.594(0.113)$ & $0.006(0.001)$ & 9.4770 \\
\hline PS1-10iw & $0.447(0.001)$ & $55326.57(1.16)$ & 0.015 & $0.378(0.557)$ & $-0.021(0.054)$ & $22.489(0.064)$ & $41.964(0.187)$ & $0.009(0.001)$ & 9.2070 \\
\hline PS1-10ix & $0.382(0.001)$ & $55338.24(0.47)$ & 0.988 & $-0.162(0.761)$ & $-0.055(0.059)$ & $22.194(0.077)$ & $41.702(0.152)$ & $0.006(0.001)$ & 7.7620 \\
\hline PS1-10iy & $0.443(0.001)$ & $55327.75(2.35)$ & & $0.289(0.718)$ & $-0.183(0.047)$ & $22.511(0.066)$ & & & 9.9070 \\
\hline PS1-10ji & $0.231(0.001)$ & $55358.81(0.39)$ & 0.681 & $-0.962(0.538)$ & $0.239(0.046)$ & $21.661(0.052)$ & $40.115(0.143)$ & $0.009(0.001)$ & 9.8820 \\
\hline PS1-10jk & $0.430(0.001)$ & $55356.45(0.84)$ & 0.037 & $1.602(0.846)$ & $-0.034(0.044)$ & $22.425(0.065)$ & $42.113(0.158)$ & $0.005(0.001)$ & 8.1620 \\
\hline PS1-10j1 & $0.530(0.001)$ & $55353.71(1.06)$ & 0.993 & $0.891(0.865)$ & $0.026(0.051)$ & $22.649(0.059)$ & $42.047(0.223)$ & $0.010(0.002)$ & 9.0020 \\
\hline PS1-10jp & $0.387(0.001)$ & $55367.83(0.74)$ & 0.680 & $-0.679(0.666)$ & $0.031(0.056)$ & $22.199(0.060)$ & $41.358(0.181)$ & & 11.3700 \\
\hline PS1-10jt & $0.336(0.001)$ & $55352.24(0.65)$ & & $-0.575(0.544)$ & & $22.200(0.059)$ & $41.013(0.141)$ & $0.006(0.001)$ & 9.8320 \\
\hline PS1-10ju & $0.323(0.001)$ & $55348.77(0.55)$ & 0.853 & $0.233(0.480)$ & $0.045(0.054)$ & $21.919(0.065)$ & $41.162(0.153)$ & 0.009 & 9.8020 \\
\hline PS1-10jv & $0.360(0.001)$ & $55355.73(0.45)$ & 0.294 & $0.539(0.468)$ & $-0.002(0.034)$ & $21.850(0.041)$ & $41.288(0.123)$ & $0.008(0.001)$ & 8.4520 \\
\hline PS1-10jw & $0.360(0.001)$ & $55358.98(0.57)$ & 0.195 & $1.023(0.585)$ & $-0.067(0.040)$ & $21.765(0.052)$ & $41.479(0.142)$ & $0.006(0.001)$ & 10.1400 \\
\hline PS1-10jz & $0.550(0.001)$ & $55354.50(0.99)$ & 0.486 & $0.518(0.819)$ & $-0.070(0.051)$ & $22.868(0.066)$ & $42.518(0.206)$ & $0.006(0.001)$ & 10.4500 \\
\hline PS1-10kc & $0.347(0.001)$ & $55358.40(0.49)$ & 0.424 & $0.447(0.597)$ & $0.104(0.044)$ & $22.058(0.052)$ & $41.143(0.152)$ & $0.006(0.001)$ & 10.4400 \\
\hline PS1-10kd & $0.331(0.001)$ & $55355.20(1.08)$ & 0.401 & $1.632(0.883)$ & $0.188(0.055)$ & $22.415(0.067)$ & $41.398(0.199)$ & $0.007(0.001)$ & 11.0800 \\
\hline PS1-10kf & $0.450(0.001)$ & $55370.40(0.89)$ & 0.845 & $0.759(0.978)$ & $0.016(0.046)$ & $22.411(0.062)$ & $41.823(0.176)$ & $0.013(0.002)$ & 9.4470 \\
\hline PS1-10kl & $0.443(0.001)$ & $55367.55(0.67)$ & 0.337 & $0.163(0.605)$ & $0.046(0.047)$ & $22.592(0.051)$ & $41.823(0.164)$ & $0.008(0.001)$ & 9.3070 \\
\hline PS1-10kv & $0.530(0.001)$ & $55364.55(0.54)$ & 0.003 & $0.441(0.455)$ & $-0.168(0.040)$ & $22.566(0.041)$ & $42.521(0.146)$ & $0.011(0.002)$ & 9.5170 \\
\hline PS1-10nq & $0.036(0.001)$ & $55351.19(1.90)$ & 0.997 & $2.259(1.798)$ & $0.147(0.070)$ & $16.983(0.079)$ & $36.183(0.263)$ & $0.017(0.003)$ & 8.5020 \\
\hline PS1-10nu & $0.064(0.001)$ & $55427.35(0.16)$ & 0.979 & $-1.115(0.126)$ & $-0.115(0.039)$ & $17.969(0.043)$ & $37.537(0.127)$ & $0.022(0.004)$ & 7.1120 \\
\hline PS1-10acx & $0.350(0.001)$ & $55438.28(0.41)$ & 0.898 & $1.407(0.421)$ & $-0.025(0.032)$ & $21.654(0.039)$ & $41.285(0.114)$ & $0.006(0.001)$ & 9.6420 \\
\hline PS1-10aeq & $0.066(0.001)$ & $55444.20(0.51)$ & 0.981 & $-0.162(0.124)$ & $0.230(0.037)$ & $18.699(0.047)$ & $37.297(0.118)$ & $0.006(0.001)$ & $\cdots$ \\
\hline PS1-10agw & $0.329(0.005)$ & $55462.21(0.27)$ & 0.994 & $-0.833(0.315)$ & $-0.156(0.037)$ & $21.620(0.057)$ & $41.357(0.114)$ & $0.058(0.009)$ & 10.6600 \\
\hline PS1-10ahk & $0.430(0.001)$ & $55473.49(0.67)$ & 0.742 & $-0.115(0.529)$ & $-0.162(0.055)$ & $22.160(0.072)$ & $42.016(0.169)$ & $0.017(0.003)$ & 9.4120 \\
\hline PS1-10ahl & $0.634(0.001)$ & $55466.70(0.86)$ & 0.888 & $-0.105(0.834)$ & $-0.214(0.066)$ & $23.023(0.085)$ & $43.048(0.252)$ & $0.087(0.014)$ & 9.8020 \\
\hline PS1-10axm & $0.509(0.005)$ & $55484.75(0.78)$ & 0.937 & $0.413(0.715)$ & $-0.040(0.054)$ & $22.494(0.065)$ & $42.036(0.198)$ & $0.023(0.004)$ & 9.7720 \\
\hline PS1-10ayb & $0.140(0.001)$ & $55494.51(0.18)$ & 0.998 & $-0.894(0.254)$ & $-0.022(0.037)$ & $19.805(0.049)$ & $39.105(0.109)$ & $0.009(0.001)$ & 10.8800 \\
\hline PS1-10bji & $0.148(0.001)$ & $55507.46(0.25)$ & 0.940 & $0.591(0.299)$ & $0.007(0.033)$ & $19.706(0.041)$ & $39.123(0.110)$ & $0.009(0.001)$ & 9.1820 \\
\hline PS1-10bjn & $0.289(0.005)$ & $55495.77(0.41)$ & 0.903 & $1.492(0.495)$ & $-0.038(0.042)$ & $21.255(0.055)$ & $40.942(0.160)$ & $0.045(0.007)$ & 10.8600 \\
\hline PS1-10bjz & $0.309(0.005)$ & $55514.01(0.47)$ & 0.024 & $0.338(0.618)$ & $-0.132(0.066)$ & $21.250(0.094)$ & $41.074(0.143)$ & $0.029(0.005)$ & 12.2600 \\
\hline PS1-10bkf & $0.173(0.001)$ & $55506.74(0.54)$ & 0.946 & $-0.917(0.456)$ & $0.058(0.036)$ & $20.836(0.046)$ & $39.877(0.127)$ & $0.020(0.003)$ & 9.8470 \\
\hline PS1-10bki & $0.431(0.001)$ & $55518.45(0.64)$ & 0.051 & $-0.549(0.502)$ & $-0.108(0.045)$ & $22.528(0.054)$ & $42.152(0.154)$ & $0.023(0.004)$ & 11.6400 \\
\hline PS1-10blh & $0.318(0.001)$ & $55522.88(0.50)$ & 0.991 & $0.319(0.586)$ & $-0.089(0.058)$ & $21.534(0.100)$ & $41.218(0.158)$ & $0.018(0.003)$ & 9.4570 \\
\hline PS1-10blp & $0.217(0.001)$ & $55521.14(1.75)$ & 0.832 & $-0.568(1.090)$ & $-0.092(0.087)$ & $20.324(0.096)$ & $39.892(0.303)$ & $0.006(0.001)$ & 11.1300 \\
\hline PS1-10bls & $0.276(0.001)$ & $55521.40(0.43)$ & 0.951 & $0.830(0.428)$ & $0.016(0.043)$ & $21.455(0.059)$ & $40.875(0.140)$ & $0.006(0.001)$ & 9.4320 \\
\hline PS1-10bmb & $0.359(0.005)$ & $55517.95(1.09)$ & 0.994 & $-1.387(1.125)$ & $-0.215(0.224)$ & $21.453(0.228)$ & $41.303(0.639)$ & $0.060(0.010)$ & 9.5770 \\
\hline
\end{tabular}




\begin{tabular}{|c|c|c|c|c|c|c|c|c|c|}
\hline & & & & & led) & & & & \\
\hline PS1 Name & $z_{\mathrm{cmb}}$ & $t_{\text {peak }}$ & $P_{\text {fit }}$ & $x_{1}$ & $c$ & $m_{B}$ & $\mu$ & $E(B-V)$ & $\begin{array}{l}\text { Host } \\
\text { Mass }\end{array}$ \\
\hline PS1-10bmk & $0.103(0.001)$ & $55535.72(0.49)$ & 0.841 & $-0.123(0.612)$ & $0.215(0.061)$ & $19.432(0.110)$ & $38.080(0.170)$ & $0.009(0.001)$ & 10.9200 \\
\hline PS1-10byq & $0.209(0.005)$ & $55543.88(0.51)$ & 0.896 & $1.539(0.638)$ & $-0.123(0.066)$ & $20.224(0.116)$ & $40.186(0.191)$ & $0.017(0.003)$ & 9.9120 \\
\hline PS1-10byr & $0.239(0.005)$ & $55534.67(1.45)$ & 0.802 & $-1.647(1.979)$ & $-0.099(0.075)$ & $21.129(0.083)$ & $40.569(0.276)$ & $0.063(0.011)$ & $\ldots$ \\
\hline PS1-10bzo & $0.381(0.001)$ & $55555.25(0.47)$ & 0.087 & $-0.198(0.506)$ & $-0.044(0.041)$ & $22.208(0.056)$ & $41.676(0.150)$ & $0.022(0.003)$ & 9.3870 \\
\hline PS1-10bzp & $0.541(0.001)$ & $55547.91(1.38)$ & 0.531 & $0.034(0.881)$ & $0.013(0.077)$ & $22.956(0.076)$ & $42.274(0.287)$ & $0.020(0.003)$ & 10.7000 \\
\hline PS1-10bzt & $0.421(0.001)$ & $55553.35(0.70)$ & 0.163 & $-0.462(0.664)$ & $0.015(0.058)$ & $22.517(0.063)$ & $41.759(0.212)$ & $0.022(0.004)$ & 9.5270 \\
\hline PS1-10bzu & $0.351(0.001)$ & $55552.03(0.74)$ & 0.940 & $-1.348(0.590)$ & $0.241(0.061)$ & $22.527(0.079)$ & $40.922(0.174)$ & $0.027(0.004)$ & 11.3700 \\
\hline PS1-10bzy & $0.200(0.001)$ & $55560.56(0.37)$ & 0.955 & $-0.232(0.290)$ & $0.035(0.044)$ & $20.745(0.054)$ & $39.954(0.147)$ & $0.009(0.001)$ & 9.3070 \\
\hline PS1-10cad & $0.270(0.005)$ & $55555.32(0.43)$ & 0.508 & $0.532(0.352)$ & $-0.029(0.036)$ & $21.051(0.049)$ & $40.575(0.1$ & $0.006(0.001)$ & 9.5020 \\
\hline PS1-10cay & $0.250(0.005)$ & $55557.62(0.88)$ & 0.995 & $2.013(0.680)$ & $0.178(0.078)$ & $21.297(0.105)$ & $40.364(0.248)$ & $0.009(0.001)$ & 10.3800 \\
\hline PS1-10cbb & $0.220(0.005)$ & $55551.35(0.95)$ & 0.545 & $-1.554(0.721)$ & $0.156(0.082)$ & $21.270(0.098)$ & $39.909(0.243)$ & $0.008(0.001)$ & 11.3000 \\
\hline PS1-10cbu & $0.401(0.001)$ & $55569.62(0.96)$ & 0.648 & $1.281(1.057)$ & $-0.051(0.061)$ & $22.099(0.090)$ & $41.798(0.227)$ & $0.020(0.003)$ & 11.4100 \\
\hline PS1-11e & $0.321(0.001)$ & $55565.32(0.85)$ & 0.002 & $-1.634(0.554)$ & $0.067(0.064)$ & $22.207(0.076)$ & $41.121(0.189)$ & $0.020(0.003)$ & 10.3000 \\
\hline PS1-11p & $0.481(0.001)$ & $55570.58(0.79)$ & 0.483 & $0.677(0.641)$ & $-0.074(0.053)$ & $22.608(0.058)$ & $42.296(0.183)$ & $0.019(0.003)$ & 8.9970 \\
\hline PS1-11s & $0.401(0.001)$ & $55561.99(0.70)$ & 0.765 & $0.584(0.588)$ & $0.110(0.052)$ & $22.409(0.056)$ & $41.494(0.216)$ & $0.015(0.002)$ & 11.2000 \\
\hline PS1-11t & $0.451(0.001)$ & $55570.93(0.49)$ & 0.488 & $0.539(0.422)$ & $-0.171(0.038)$ & $22.179(0.045)$ & $42.158(0.133)$ & $0.014(0.002)$ & 10.4100 \\
\hline PS1-11w & $0.174(0.001)$ & $55568.03(0.30)$ & 0.995 & $-0.484(0.213)$ & $-0.060(0.031)$ & $20.009(0.038)$ & $39.487(0.103)$ & $0.007(0.001)$ & 8.9020 \\
\hline PS1-11aj & $0.107(0.001)$ & $55548.56(1.28)$ & 0.850 & $-1.576(0.390)$ & $-0.047(0.076)$ & $19.532(0.074)$ & $38.818(0.220)$ & $0.016(0.003)$ & 11.2300 \\
\hline PS1-11at & $0.321(0.001)$ & $55571.24(0.51)$ & 0.665 & $-2.217(0.523)$ & $0.068(0.064)$ & $21.868(0.077)$ & $40.696(0.178)$ & $0.014(0.002)$ & 10.6200 \\
\hline PS1-11bg & $0.330(0.001)$ & $55583.99(0.37)$ & 0.428 & $-0.200(0.398)$ & $-0.068(0.035)$ & $21.725(0.049)$ & $41.270(0.112)$ & $0.019(0.003)$ & 10.7200 \\
\hline PS1-11bh & $0.351(0.001)$ & $55580.52(0.34)$ & 0.171 & $0.175(0.441)$ & $-0.090(0.041)$ & $21.791(0.060)$ & $41.457(0.1$ & $0.016(0.003)$ & 10.8000 \\
\hline PS1-11bk & $0.161(0.001)$ & $55584.81(0.31)$ & 0.061 & $-0.432(0.245)$ & $0.045(0.046)$ & $20.258(0.045)$ & $39.407(0.141)$ & $0.019(0.003)$ & 12.7200 \\
\hline PS1-11br & $0.300(0.005)$ & $55577.12(3.02)$ & 0.994 & $-0.453(1.387)$ & $-0.240(0.020)$ & $21.226(0.073)$ & $41.286(0.236)$ & $0.009(0.001)$ & $\ldots$ \\
\hline PS1-11cn & $0.249(0.005)$ & $55583.37(0.52)$ & 0.680 & $0.442(0.470)$ & $-0.163(0.035)$ & $20.669(0.053)$ & $40.608(0.130)$ & $0.022(0.003)$ & 10.2500 \\
\hline PS1-11co & $0.230(0.005)$ & $55590.64(1.04)$ & 0.801 & $-0.321(1.190)$ & $-0.068(0.065)$ & $20.871(0.087)$ & $40.400(0.179)$ & $0.007(0.001)$ & 9.2370 \\
\hline PS1-11fi & $0.082(0.001)$ & $55596.22(0.03)$ & 0.664 & $-1.185(0.272)$ & $0.141(0.042)$ & $18.998(0.060)$ & $37.736(0.148)$ & $0.008(0.001)$ & $\cdots$ \\
\hline PS1-11iv & $0.294(0.001)$ & $55595.98(1.44)$ & 0.889 & $0.158(0.468)$ & $-0.086(0.041)$ & $21.361(0.048)$ & $41.014(0.154)$ & $0.011(0.002)$ & 9.4320 \\
\hline PS1-11jo & $0.331(0.001)$ & $55607.51(0.43)$ & 0.242 & $0.622(0.445)$ & $-0.071(0.038)$ & $21.674(0.055)$ & $41.344(0.153)$ & $0.014(0.002)$ & $\ldots$ \\
\hline PS1-11mq & $0.210(0.001)$ & $55639.09(0.25)$ & 0.926 & $1.405(0.234)$ & $-0.101(0.029)$ & $20.225(0.037)$ & $40.100(0.110)$ & $0.007(0.001)$ & 8.4520 \\
\hline PS1-11mz & $0.102(0.001)$ & $55642.57(0.14)$ & 0.971 & $0.685(0.174)$ & $0.023(0.029)$ & $18.951(0.041)$ & $38.328(0.106)$ & $0.013(0.002)$ & 10.1800 \\
\hline PS1-11sk & $0.271(0.001)$ & $55678.26(0.26)$ & 0.577 & $0.026(0.200)$ & $0.115(0.029)$ & $21.582(0.036)$ & $40.574(0.101)$ & $0.016(0.003)$ & 9.2420 \\
\hline PS1-11uo & $0.310(0.001)$ & $55686.17(0.32)$ & 0.892 & $0.608(0.358)$ & $-0.089(0.034)$ & $21.384(0.045)$ & $41.108(0.118)$ & $0.006(0.001)$ & 11.3700 \\
\hline PS1-11uw & $0.300(0.001)$ & $55681.60(0.44)$ & & & $-0.156(0.0$ & $21.124(0.049)$ & $41.051(0.1$ & $0.008(0.0$ & 10.7500 \\
\hline PS1-11wv & $0.133(0.001)$ & $55686.57(0.10)$ & 0.823 & $0.257(0.140)$ & $-0.124(0.028)$ & $19.109(0.037)$ & $38.898(0.099)$ & $0.012(0.002)$ & 11.3000 \\
\hline PS1-11xc & $0.328(0.001)$ & $55691.25(0.60)$ & 0.638 & $2.481(1.102)$ & $0.161(0.066)$ & $22.130(0.132)$ & $41.316(0.283)$ & $0.008(0.001)$ & 12.8200 \\
\hline PS1-11xw & $0.271(0.001)$ & $55700.27(0.33)$ & 0.410 & $-1.072(0.311)$ & $-0.077(0.033)$ & $21.205(0.041)$ & $40.656(0.117)$ & $0.010(0.002)$ & 11.8500 \\
\hline PS1-11yr & $0.531(0.001)$ & $55714.70(0.07)$ & 0.965 & $1.341(1.597)$ & $-0.128(0.063)$ & $22.950(0.072)$ & $42.903(0.282)$ & $0.010(0.002)$ & 8.5420 \\
\hline PS1-11zd & $0.100(0.001)$ & $55701.26(0.75)$ & 0.979 & $0.596(0.211)$ & $-0.018(0.030)$ & $18.907(0.051)$ & $38.404(0.111)$ & $0.010(0.002)$ & 10.1700 \\
\hline PS1-11zg & $0.370(0.001)$ & $55712.81(1.15)$ & 0.368 & $-0.136(1.828)$ & $-0.068(0.057)$ & $21.913(0.062)$ & $41.466(0.274)$ & $0.007(0.001)$ & 10.6400 \\
\hline PS1-11zu & $0.360(0.001)$ & $55711.46(0.72)$ & 0.970 & $-0.639(0.379)$ & $-0.002(0.046)$ & $22.035(0.048)$ & $41.306(0.157)$ & $0.008(0.001)$ & 10.2400 \\
\hline PS1-11zv & $0.350(0.001)$ & $55718.21(0.45)$ & 0.831 & $0.103(0.356)$ & $-0.060(0.056)$ & $21.727(0.075)$ & $41.289(0.180)$ & $0.008(0.001)$ & 10.4400 \\
\hline PS1-11zw & $0.423(0.001)$ & $55711.38(1.70)$ & 0.890 & $-0.471(0.977)$ & $-0.060(0.118)$ & $22.784(0.090)$ & $42.265(0.419)$ & $0.009(0.001)$ & 11.4700 \\
\hline PS1-11abm & $0.321(0.001)$ & $55727.76(0.96)$ & 0.872 & $-1.739(0.897)$ & $-0.123(0.058)$ & $21.777(0.075)$ & $41.282(0.194)$ & $0.010(0.002)$ & 9.8970 \\
\hline PS1-11aea & $0.300(0.001)$ & $55738.26(0.55)$ & 0.846 & $-0.873(0.249)$ & $-0.036(0.032)$ & $21.788(0.046)$ & $41.138(0.111)$ & $0.007(0.001)$ & 9.4370 \\
\hline PS1-11aij & $0.510(0.001)$ & $55774.44(0.51)$ & 0.779 & $0.861(0.444)$ & $-0.020(0.040)$ & $22.672(0.040)$ & $42.213(0.145)$ & $0.006(0.001)$ & 8.7770 \\
\hline PS1-11ajs & $0.229(0.005)$ & $55784.21(0.18)$ & 0.704 & $-0.128(0.220)$ & $0.113(0.034)$ & $21.340(0.047)$ & $40.317(0.121)$ & $0.039(0.006)$ & 8.8470 \\
\hline PS1-11ala & $0.369(0.005)$ & $55795.13(0.30)$ & 0.168 & $0.285(0.262)$ & $-0.103(0.031)$ & $21.666(0.038)$ & $41.389(0.118)$ & $0.032(0.005)$ & 11.5000 \\
\hline PS1-11alv & $0.144(0.001)$ & $55810.78(0.14)$ & 0.990 & $0.981(0.160)$ & $-0.004(0.030)$ & $19.686(0.047)$ & $39.192(0.101)$ & $0.054(0.009)$ & 10.7300 \\
\hline
\end{tabular}

Notes. Fit parameters of all SNe Ia in the Pan-STARRS1 spectroscopic sample that passed all cuts. The redshift is corrected for coherent flow effects. $t_{\text {peak }}$ is the fitted time of $B$-band maximum $m_{B}$. $P_{\text {fit }}$ is the SALT2 fit probability. $x_{1}, c, m_{B}$, and $\mu$ are the parameters as defined in Equation (2). The Milky Way Galaxy reddening is given in the direction of the SN by Schlegel et al. (1998) and corrected by 6\%-14\% as described in S14. The host galaxy mass given as $\log _{10} M_{\text {host }}$.

color and uncorrected distance residuals. The second uses the assumption that the observed color is the combination of intrinsic SN color, photometric errors, and reddening due to dust. The former is less model dependent, while the latter is more physically motivated.

Ultimately, these choices have small, but noticeable consequences for cosmological inferences. Kessler et al. (2009a) made an in-depth analysis of the different light curve fitters, and found that in general they agree reasonably well given the same assumptions. Currently the most widely used light curve fitter is SALT2 (Guy et al. 2007), and we use this fitter in our analysis. SALT2 explicitly matches the observations in the filters of any given survey to integrals of the warped model spectra for those passbands. It treats the color of SNe Ia entirely empirically, and is used to find an overall relation between luminosity and color. S14 explores whether the linear models used by SALT2 to describe the color and stretch-luminosity relation are adequate to fit the data. 


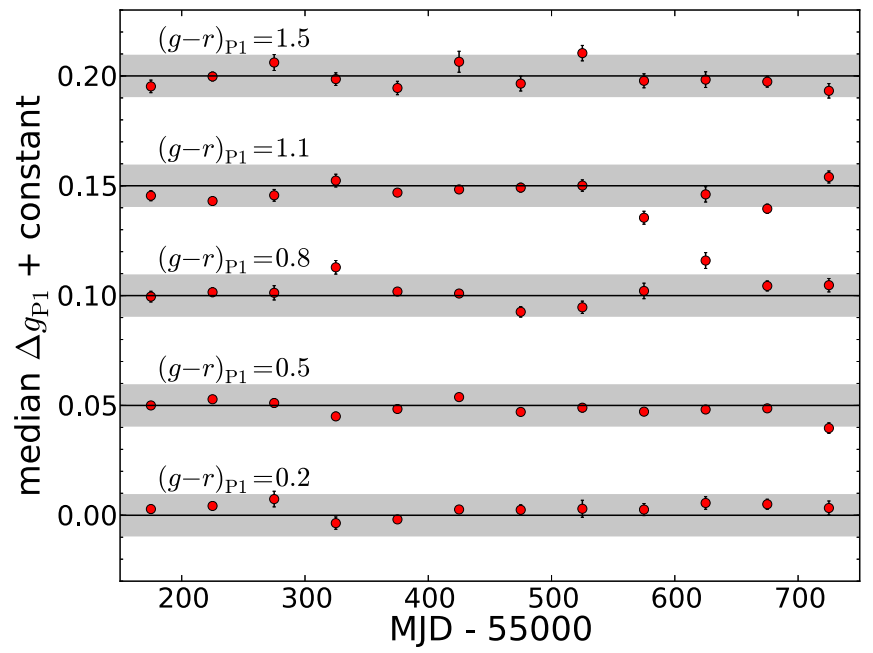

Figure 11. Median difference between the average magnitude of stars and their associated detections at different epochs for color bins of $(g-r)_{P 1}$ in $[0.2,0.5$, $0.8,1.1,1.5]$. The size of the color bins is $0.1 \mathrm{mag}$, and only stars with $r_{\mathrm{P} 1}<19$ were used. For clarity, offsets in steps of 0.05 are added to $\Delta g_{\mathrm{P} 1}$, and the gray shaded bars indicate $\mathrm{a} \pm 1 \%$ level deviation. No long-term variation is apparent above \pm 3 mmag, which we assign as the systematic uncertainty.

(A color version of this figure is available in the online journal.)

We use the most up-to-date published version of SALT2 (Guy et al. 2010) implemented in SNANA ${ }^{25}$ (Kessler et al. 2009b). We transform our light curve fit parameter into distances using the Tripp formula (Tripp 1998)

$$
\mu_{B}=m_{B}-M+\alpha x_{1}-\beta c,
$$

where $\mu_{B}$ is the distance modulus, $m_{B}$ is the peak $B$-band brightness, $x_{1}$ is a light curve shape parameter, and $c$ is a color parameter. The parameters $\alpha, \beta$, and $M$ are nuisance parameters. $\alpha$ is determined by the relation between luminosity and stretch while $\beta$ is determined by the relation between luminosity and color. $M$ is the absolute $B$-band magnitude of a fiducial SN Ia with $x_{1}=0$ and $c=0$. Motivated by Schlafly \& Finkbeiner (2011), we make one modification to SALT2 by replacing the "CCM" (Cardelli et al. 1989) Milky Way Galaxy (MWG) reddening law with that from Fitzpatrick (1999). Schlafly \& Finkbeiner (2011) also finds that the MWG extinction values from Schlegel et al. (1998) are overestimated by 6\%-14\%. We therefore correct our extinction values accordingly. These changes are explained further in Section 7 in S14.

We present in Tables 7 and 8 the SALT2 parameters for the entire set of cosmologically useful SNe Ia from the PS1 plus low $z$ sample (PS1+lz). The cuts that are used to remove SNe Ia from the cosmological sample are described in the second part of this section. Distributions of the SALT2 fit parameter are shown in Figure 12.

Discrepancies in the $x_{1}$ and $c$ distributions between the PS1 and low- $z$ samples are likely due to selection effects. Most lowredshift $\mathrm{SNe}$ in our sample were discovered in surveys that target specific nearby galaxies (Li et al. 2011). These surveys are capable of detecting SNe Ia with more extinction than the untargeted sample from PS1. We use stringent cuts on the light curve properties, as described below, that pass only about one half of the low- $z$ sample but four-fifths of the PS1 sample.

\footnotetext{
25 SNANA_v10_23
}
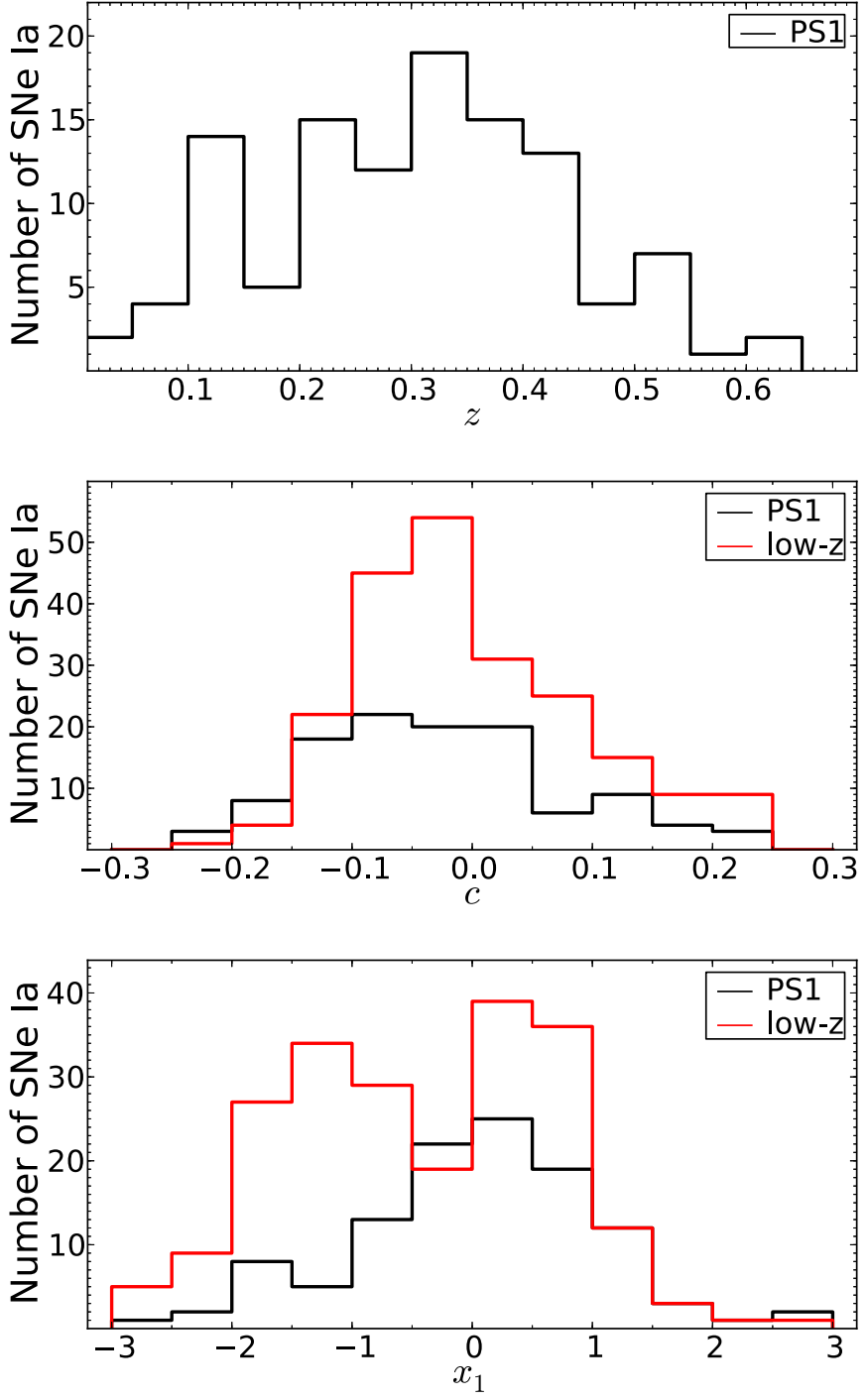

Figure 12. Histograms of redshift, color, and stretch for the Pan-STARRS1 (black solid) and low-redshift (red dashed) samples. The first bin of the lowredshift sample has 222 light curves from 197 SNe Ia. For the samples presented, both color and stretch cuts were applied (see Section 7.2), but not the minimum redshift cut.

(A color version of this figure is available in the online journal.)

\subsection{Sample Cuts}

The PS1 survey spectroscopically confirmed $146 \mathrm{SNe}$ Ia during the first $1.5 \mathrm{yr}$ in the MDFs. This was only a very small fraction of the $\sim 1700$ transients with SN Ia-like light curves. While it would be ideal to use all spectroscopically confirmed PS1 SNe Ia to constrain cosmological parameters, we use sharp quality cuts to improve the analysis. We require that every SNe Ia has adequate light curve coverage to properly measure a distance and that it has a light curve fit, redshift, and Milky Way extinction that limits systematic bias in the distance. Applying these cuts, the cosmological analysis of Section 8 employs 113 SNe Ia from the initial sample of 146 spectroscopically confirmed PS1 objects.

For our low- $z$ SNe Ia, we use the following SNe Ia samples: we denote JRK07 as the compilation of SNe Ia collected by Calán/Tololo (Hamuy et al. 1996a, 29 SNe), CfA1 (Riess et al. 1999, 22 SNe), CfA2 (Jha et al. 2006, 44 SNe), and other sources (Jha et al. 2007, $28 \mathrm{SNe}$ ). We also use the SNe Ia compiled 
Table 9

Contributions and Cuts of the Different Samples

\begin{tabular}{|c|c|c|c|c|c|c|c|c|}
\hline Set & Total & Final & $\begin{array}{c}\text { Initial } \\
\text { Cut }\end{array}$ & $\begin{array}{l}\text { First Pass } \\
\quad \text { Cut }\end{array}$ & $\begin{array}{c}\text { Fit Not } \\
\text { Conv. }\end{array}$ & $P<0.001$ & $\begin{array}{l}\left|x_{1}\right|>3.0 \\
|c|>0.25\end{array}$ & Chauveneut \\
\hline JRK07 & 133 & 49 & 30 & 34 & 1 & 12 & 7 & 0 \\
\hline CFA3 & 185 & 85 & 8 & 70 & 0 & 7 & 15 & 0 \\
\hline CFA4 & 94 & 43 & 8 & 26 & 3 & 8 & 5 & 1 \\
\hline CSP & 85 & 45 & 5 & 13 & 0 & 14 & 7 & 1 \\
\hline PS1 & 147 & 112 & 10 & 18 & 2 & 1 & 4 & 0 \\
\hline
\end{tabular}

Notes. Breakdown of the number of SNe Ia that were cut from the different sets. The different sets are defined in Section 7.2.

more recently by CfA3 (Hicken et al. 2009a, 185 SNe), CSP (Contreras et al. 2010, 85 SNe), and CfA4 (Hicken et al. 2012, $94 \mathrm{SNe}$ ). After applying our cuts, the low- $z$ sample is trimmed from 497 to 222 light curves from $197 \mathrm{SNe}$ Ia. Table 9 shows the effect of each cut on the different samples.

Below, we detail the cuts. We apply these criteria in three steps. Initial criteria do not require any light curve fitting. Light curves that do not meet these standards are eliminated. Firstpass criteria are determined from polynomial fits to the light curves. Light curves that pass these tests are subjected to our final criteria, which use the output of the full SALT2 light curve fit.

\section{Initial criteria:}

1. Unambiguous spectroscopic classification as a SN Ia.

2. Not a Iax- or 91bg-like SN Ia.

3. Outside the central $0.3 \mathrm{deg}$ of the MDF (PS1 only).

4. $z>0.01$.

5. Galactic reddening along the line of sight of $E(B-V)_{\mathrm{MWG}}<0.5 \mathrm{mag}$.

6. Measurements in 2 or more filters with $S / N \geqslant 5$.

First-pass criteria:

7. At least 1 measurement with -10 days $<t<+5$ days.

8. At least 1 measurement with +5 days $<t<+20$ days.

9. 5 or more measurements with -10 days $<t<+35$ days.

10. 2 or more filters with a measurement between -8 days $<$ $t<+10$ days.

Final criteria:

11. Light curve fit converges.

12. $\mathcal{P}_{\text {fit }}>0.001$, where $\mathcal{P}_{\text {fit }}$ is the SALT2 light-curve fit probability based on the $\chi^{2}$ per degree of freedom.

13. $-0.25<c<0.25$ or $-3.0<x_{1}<3.0$.

14. Outlier rejection (Chauvenet's criterion from Taylor 1997; $4 \sigma)$

\subsubsection{Initial Cuts}

We require spectroscopic confirmation of all $\mathrm{SNe}$ Ia used in our cosmological analysis. We follow a method similar to that presented by Foley et al. (2009b) to determine the classification of each potential SN Ia. Briefly, we use SNID (Blondin \& Tonry 2007) to match a SN spectrum with a library of high-S/N spectra. SNID provides a quantitative assessment that a particular $\mathrm{SN}$ is of a given class at a given redshift. Some redshifts come from host galaxy emission lines. Each SN is ultimately classified by spectroscopists: co-authors R.C. and R.J.F. SNe with ambiguous classifications are eliminated. Although the classification is subjective, almost every SN in the PS1 cosmology sample has a spectrum of high quality and it is unlikely that any non-SNe Ia have leaked into this analysis. Similarly, there should be no catastrophic failures in the determination of the redshifts.

We specifically exclude $\mathrm{SNe}$ of the "Iax" subclass (Foley et al. 2013) from all samples. Although this mostly affects the low-redshift samples, SN 2009ku, a SN Iax discovered by PS1 (Narayan et al. 2011), is removed from our final sample. We also exclude PS1-11yj, a SN 1991bg-like object at $z=0.107$. We decided not to include this peculiar SN because it has not yet been shown whether this subclass of $\mathrm{SNe}$ Ia can be well represented by the training sample and has been excluded in some past surveys (e.g., Hicken et al. 2009b). The three SNe Ia (PS1-10f, PS1-11yu, PS1-11ams) that are within $0.3 \mathrm{deg}$ of the field center are excluded, since the absolute photometric calibration is uncertain due to strong PSF variation (see Section 6.2).

We exclude all SNe with $z<0.01$ to avoid objects affected by departures from Hubble's Law due to bulk flows or a regional Hubble bubble. We exclude SNe with a Milky Way reddening $E(B-V)_{\mathrm{MWG}}>0.5 \mathrm{mag}$ to avoid introducing any substantial error due to the extinction correction. These cuts affect only the low-redshift sample, and S14 provides a detailed explanation of the effects of these cuts.

\subsubsection{First-pass Cuts}

We require high-quality light curves that when fit result in accurate distance measurements. One requirement is that the $\mathrm{S} / \mathrm{N}$ is sufficiently large in a minimum number of observations. A large subset of requirements can be considered "coverage" cuts, where each light curve must have observations in certain phase ranges with certain filters. Finally, we require that the resulting light curve fit parameters fall within ranges known to have low distance biases from simulations. We outline the cuts below.

For our sample, we require that there be measurements in at least two bands with $\mathrm{S} / \mathrm{N}>5$. This requirement does not affect the low-redshift or PS1 spectroscopic sample; however, it does reflect our detection limits, and is a necessary requirement for simulating the survey.

The light curve cuts applied to the PS1 sample are taken from Guy et al. (2010). Most requirements are expressed in terms of the rest-frame phase $t=\left(t_{\mathrm{obs}}-t_{\max }\right) /(1+z)$. While Kessler et al. (2009a) required at least one measurement with $t<0$ days, Guy et al. (2010) found that a more flexible requirement of needing one measurement in the range of -8 days $<t<5$ days provided a similar constraint. Using simulations of the PS1 and low- $z$ samples from S14, we find that SNe Ia that pass the Guy et al. (2010) cut, but not the Kessler et al. (2009a) cut only introduce a very small bias of $0.2 \%$ into the distance modulus. This bias increases to $0.4 \%$ for redshifts larger than 
0.5. However, there are no high-redshift SNe Ia in our PS1 sample that pass the Guy et al. (2010) but not the Kessler et al. (2009a) cut.

\subsubsection{Final Cuts}

We require that the light curve fit converges and has a SALT2 light curve fit probability $\mathcal{P}_{\text {fit }}>0.001$ based on the $\chi^{2}$ per degree of freedom. We remove all $\mathrm{SNe}$ Ia in our sample with $|c|>0.25$ or $\left|x_{1}\right|>3$. Colors or stretch values that deviate far from zero are not well represented in the training sample and such objects could bias the measurement of $\beta$. Where we have light curves of the same SNe from two different surveys, we take the average distance of the two so that the $\mathrm{SN}$ is only treated as a single independent data point.

Finally, when fitting cosmological parameters, we apply Chauvenet's criterion (Taylor 1997) to reject outliers, removing $\mathrm{SNe}$ for which we could expect less than half of an event in our full sample (assuming a Gaussian distribution of intrinsic luminosities). For the PS1+lz sample, this is $4 \sigma$. This criterion does not depend significantly on our choice of cosmological parameters, and thus the same $\mathrm{SNe}$ are excluded for all cosmological choices. Chauvenet's criterion removes no SNe Ia from the PS1 sample and two (SN 2004gc and SN 2008cm) from the low-z sample.

We make all data and tools used for this analysis publicly available at http://ps1sc.org/transients/.

\section{COSMOLOGICAL RESULTS}

\subsection{Luminosity Distance Measurements}

The ultimate goal of this analysis is to put constraints on the cosmological parameters $\Omega_{\mathrm{M}}, \Omega_{\Lambda}$, and $w$. We first transform the SALT2 fit parameters into distances using the SALT2mu program (Marriner et al. 2011), which finds the $\alpha$ and $\beta$ parameters that minimize the distance modulus residuals for a given cosmology. While there is uncertainty in whether these parameters evolve with redshift (Kessler et al. 2009a; Conley et al. 2011), here we assume that $\alpha, \beta$, and $M$ are all constant with redshift. In order for the $\chi_{v}^{2}$ of the distance residuals to be unity, an intrinsic dispersion $\sigma_{\text {int }}$ is added in quadrature to the error of each SN distance (which includes the distance error from redshift uncertainty). ${ }^{26}$ Both the intrinsic dispersion of the sample and the photometric errors of each SN distance include a dependence on the nuisance parameters and covariances between the fit parameters. The SALT2mu procedure propagates these errors and determines the values for $\alpha$ and $\beta$.

There is ongoing debate about the source of the intrinsic scatter seen in SNe Ia distances. As shown in Kessler et al. (2013) and Scolnic et al. (2014a), the determined values of $\alpha$ and $\beta$ depend on assumptions about the source of the intrinsic scatter. For the PS $1+\mathrm{lz}$ sample, $\alpha$ and $\beta$ are given in Table 10 after attributing the remaining intrinsic distance scatter to either luminosity variation or color variation. This is done after the SALT2 light curve fit includes a small amount of color variation in its model. We find that the intrinsic dispersion of the sample is $0.115 \mathrm{mag}$ if we attribute intrinsic scatter to luminosity variation and $0.025 \mathrm{mag}$ if we attribute intrinsic scatter to color variation. There is a large difference in the values of $\beta$ found for these two different assumptions: $\beta=3.13 \pm 0.12$ and $\beta=3.71 \pm 0.15$

$26 \sigma^{2}=\sigma_{\mathrm{N}}^{2}+\sigma_{\mathrm{int}}^{2}+\sigma_{\mu-z}^{2}$, where $\sigma_{\mathrm{N}}^{2}$ is the photometric error of the SN distance, $\sigma_{\mathrm{int}}^{2}$ is the intrinsic scatter, and $\sigma_{\mu-z}^{2}$ is the distance error due to redshift uncertainty.
Table 10

Effects of Choices for Intrinsic Scatter

\begin{tabular}{lcc}
\hline \hline Intrinsic Scatter & $\alpha$ & $\beta$ \\
\hline$\sigma_{\text {int }, m_{B}}=0.115$ & $0.147 \pm 0.010$ & $3.13 \pm 0.12$ \\
$\sigma_{\text {int }, c}=0.025$ & $0.141 \pm 0.010$ & $3.71 \pm 0.15$ \\
\hline
\end{tabular}

Notes. Intrinsic scatter $\sigma_{\mathrm{int}, m_{B}}$ and $\sigma_{\mathrm{int}, c}$ in the PS $1+\mathrm{lz}$ sample, and how $\alpha$ and $\beta$ vary for each method. The magnitudes of each scatter given above is such that the total reduced $\chi^{2}$ of the sample is $\sim 1.0$.

for the luminosity and color variation, respectively. The value of $\beta$ found for the color variation case is within $3 \sigma$ of a MW-like reddening law. Interestingly, the low $-z$ sample by itself pulls $\beta$ to a higher value $(\beta \sim 3.9)$ than the PS1 sample $(\beta \sim 3.0)$ when we attribute scatter to color variation. This may be due to the different selection effects in the low- $z$ and PS1 samples, or the incompleteness of the SALT2 training sample for blue colors (S14). Most likely related, the total intrinsic scatter of the PS1 distances is half as large $\left(\sigma_{\text {int }}=0.07\right)$ as that for the low $z$ sample $\left(\sigma_{\text {int }}=0.123\right)$. The scatter seen for the low $-z$ sample is larger than that found in past studies (e.g., Kessler et al. 2009a) as the assumed peculiar velocity errors in this analysis are smaller. We fix different values of $\sigma_{\text {int }}$ for our high and individual low- $z$ subsamples. These values are given in S14.

The source of intrinsic scatter is explored in the companion paper S14. To summarize briefly, we create two simulations of the PS $1+\mathrm{lz}$ samples in which one has $\beta=3.1$ and scatter is dominated by luminosity variation, and another in which $\beta$ is consistent with a MW-like value $(\beta=4.1)$ and scatter is dominated by color variation. We then find the biases in distance over the entire redshift range from subtracting our recovered distances from the simulated distances. For both simulations, we assume the scatter comes from luminosity variation alone. Since we simulate the full sample, the discrepancies between recovered and simulated distances will also be composed of the Malmquist bias at the upper limits of our redshift ranges. The differences in distance modulus correction from the two simulations can be as high as $4 \%$. To correct the distances, we take the average correction from these two simulations at each redshift. We do not choose one model or the other as there is insufficient empirical evidence to favor either model (S14). Understanding and correcting for the intrinsic variation of SNe Ia is one of the largest systematic uncertainties in our analysis. In Figure 13, we show the bias in distances for both the low $-z$ and PS1 sample. For any given redshift, we then interpolate the bias from this average correction vector. This bias is subtracted from all PS1 distance moduli.

The PS1+lz Hubble diagram with the corrected distances is shown in Figure 14. Three model universes are given: the $\Lambda$ CDM universe, a universe with $\Omega_{\mathrm{M}}=1$, and a universe with $\Omega_{\mathrm{M}}=0.3$. The distance modulus $\mu\left(H_{0}, \Omega_{\mathrm{M}}, \Omega_{\Lambda}, w, z\right)$ is found from the luminosity distance $d_{L}$ such that $\mu=5 \times \log \left(d_{L}\right)+25$. The luminosity distance is commonly expressed as

$$
\begin{aligned}
& d_{L}\left(z ; w, \Omega_{\mathrm{M}}, \Omega_{\Lambda}, H_{0}\right) \\
& =(1+z)\left|\Omega_{\mathrm{k}}\right|^{-1 / 2} \mathcal{S}_{k}\left[c\left|\Omega_{\mathrm{k}}\right|^{1 / 2} \int_{0}^{z} \frac{d z^{\prime}}{H\left(z^{\prime}\right)}\right],
\end{aligned}
$$

where the curvature density $\Omega_{\mathrm{k}} \equiv 1-\Omega_{\mathrm{M}}-\Omega_{\Lambda}$, and the function $\mathcal{S}_{k}(x)=\sin (x)$ for $\Omega_{\mathrm{k}}<0, \mathcal{S}_{k}(x)=\sinh (x)$ for $\Omega_{\mathrm{k}}>0$, and $\mathcal{S}_{k}(x)=x$ for a flat universe with $\Omega_{\mathrm{k}}=0$. Finally, the function $H(z)$ is defined as

$$
H(z)=H_{0}\left[\Omega_{\mathrm{M}}(1+z)^{3}+\Omega_{\Lambda}(1+z)^{3(1+w)}+\Omega_{\mathrm{k}}(1+z)^{2}\right]^{1 / 2} .
$$




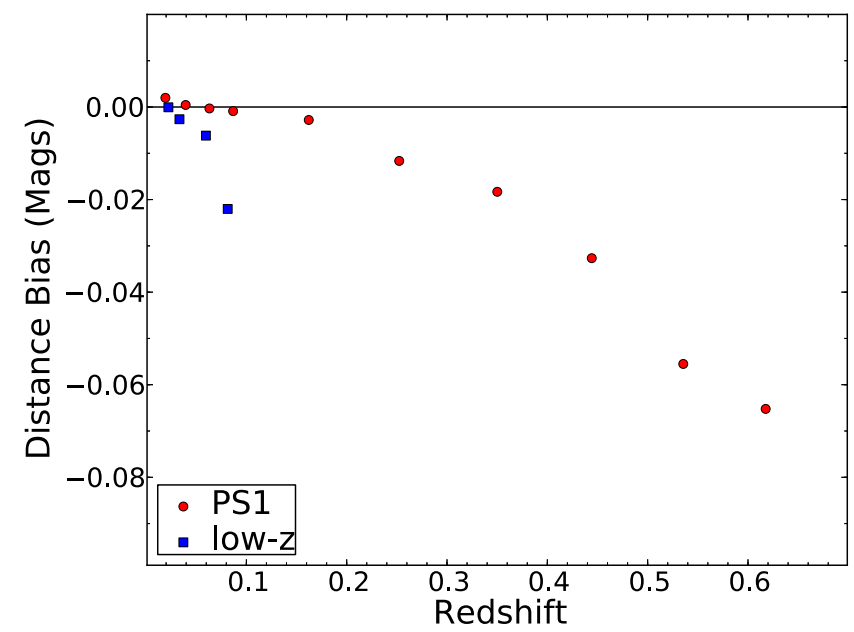

Figure 13. Distance corrections for the low $z$ and Pan-STARRS1 samples as a function of redshift.

(A color version of this figure is available in the online journal.)

Cosmological constraints can be found from the SN sample with only statistical errors by measuring the $\chi^{2}$ value for a grid of $\Omega_{\Lambda}$ versus $\Omega_{\mathrm{M}}$ values with $w=-1$ and $w$ versus $\Omega_{\Lambda}$ for a flat universe $\left(\Omega_{\Lambda}+\Omega_{\mathrm{M}}=1\right)$. The PS1 sample does not probe high enough redshifts to provide much constraining power on evolving equations of state, and we thus assume a constant $w$ and a flat universe when we determine the constraints for $w$ and $\Omega_{\mathrm{M}}$.

\subsection{Systematic Uncertainties}

We briefly summarize the systematic uncertainty analysis detailed by S14. They derive a covariance matrix for the systematic uncertainties that may be included when determining the PS1 cosmological results. The most important systematic uncertainties are due to calibration uncertainties, assumptions in the light curve fitting and selection effects.

Uncertainties in the PS1 photometric system due to calibration are given in Table 5. When combining the PS1 sample with the low $-z$ sample, we include calibration uncertainties from each of the low $-z$ samples. These uncertainties are composed of uncertainties in bandpasses and zero points as well as the uncertainty in the Landolt standards. The calibration uncertainties make up $>50 \%$ of the total systematic uncertainty of the sample. The other largest uncertainties are due to incomplete understanding of the intrinsic color of SNe Ia, selection effects and Milky Way extinction corrections. Further possible systematic uncertainties are due to dependencies of fitted distances on host galaxy properties and coherent flow effects. While we correct the redshifts of the low- $z$ SNe for coherent flow effects (Neill et al. 2007), we currently do not correct for the dependence on host galaxy properties. This adjustment is not applied
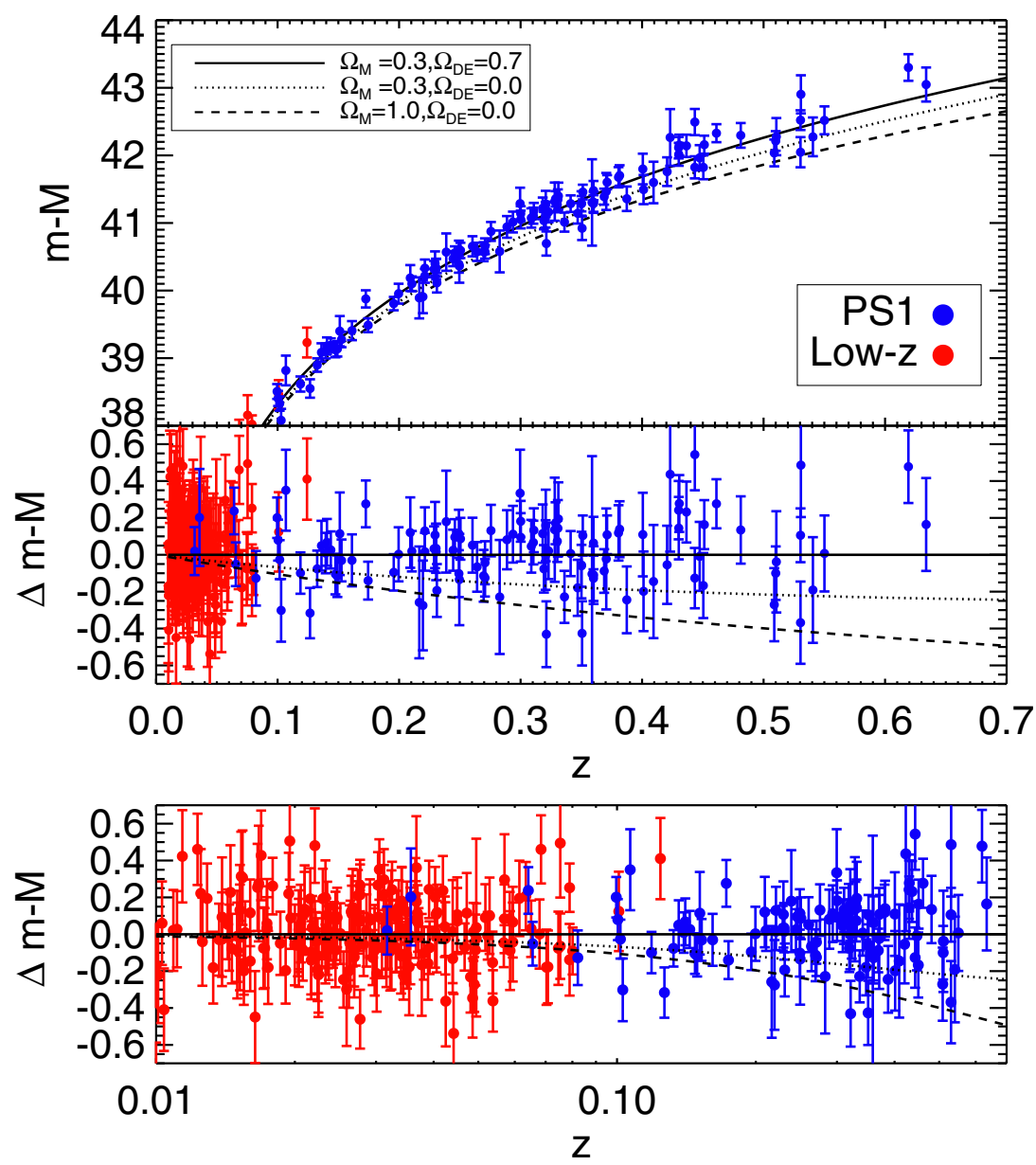

Figure 14. Hubble diagram for the combined PS1 and low-redshift sample. The bottom panel shows the difference modulus residuals vs. the logarithmic redshift in order to visualize the low- $z$ SNe Ia residuals.

(A color version of this figure is available in the online journal.) 
as the difference in Hubble residuals for low- and high-mass host galaxies is only $0.037 \pm 0.032$ (S14). For the use of future studies, we present the host galaxy masses $M_{\text {host }}$ in Table 8 . The derivation of the masses is described in S14.

Following Conley et al. (2011), the uncertainties are propagated through a systematic error matrix. We define a total uncertainty matrix $\mathbf{C}$ such that $\mathbf{C}=\mathbf{D}_{\text {stat }}+\mathbf{C}_{\text {sys }}$. The statistical matrix $\mathbf{D}_{\text {stat }}$ has only a diagonal component that includes errors from the fit parameters and intrinsic scatter. While statistical covariances may arise from errors in the SALT2 template, Conley et al. (2011) finds these to be negligible. The systematic error matrix is determined by varying a given uncertainty parameter, and then finding the difference between the original distance and a newly determined distance. While the statistical covariance matrix includes components of the error budget that can, in principle, be reduced by adding more SNe Ia to the sample, the systematic uncertainties can be reduced with improved analysis or external data. Given a vector of distance residuals of the SN sample $\Delta \mu=\mu_{B}-\mu\left(H_{0}, \Omega_{\mathrm{M}}, \Omega_{\Lambda}, w, z\right)$, then $\chi^{2}$ may be expressed as

$$
\chi^{2}=\Delta \boldsymbol{\mu}^{T} \cdot \mathbf{C}^{-1} \cdot \Delta \boldsymbol{\mu} .
$$

These constraints are shown Figure 15 and compared to the constraints from the statistical only sample. As the systematic uncertainties weight the errors of SNe differently, the best-fit values of the recovered parameters will be shifted.

\subsection{External Constraints}

To better determine cosmological parameters, we include constraints from baryon acoustic oscillations (BAOs; Blake et al. 2011), the CMB (Planck Collaboration et al. 2013), and the Hubble constant (Riess et al. 2011). In order to focus on the constraints from the PS1 sample and simplify the analysis, we do not include additional high-redshift SN Ia samples (e.g., SNLS and HST). A combination of all SNe data will be left for a future study.

We follow Planck Collaboration et al. (2013) to include constraints from other surveys, as they have made the most precise measurements on the $\mathrm{CMB}$, and gather data from all of the various BAO surveys to determine this constraint. The likelihood of cosmological parameters is found from the Markov Chains given as an extension of Planck Collaboration et al. (2013). We note that there are still unresolved calibration discrepancies between Planck and WMAP (Hinshaw et al. 2013), and the constraints from these two surveys are compared in S14.

For Planck, we use their Markov Chains for determining cosmological parameters. When we wish to explore a flat $w$ CDM model, we use their $+w$ model. When we want to examine non-flat $\Lambda \mathrm{CDM}$ models, we use their $+k$ model. For all CMB constraints, we include data from the Planck temperature power spectrum data, Planck temperature data, Planck lensing, and WMAP polarization at low multipoles. For the BAO constraint, we take data from a multitude of surveys, as shown in Table 11.

For the Hubble constraint, we follow Riess et al. (2011), which uses HST observations of Cepheid variables in the host galaxies of eight SNe Ia to calibrate the SN magnitude-redshift relation. Their best estimate of the Hubble constant is $H_{0}=$ $73.8 \pm 2.4 \mathrm{~km} \mathrm{~s}^{-1} \mathrm{Mpc}^{-1}$.

\subsection{Cosmological Parameter Constraints}

Using the set of distances and redshifts for the PS1+lz sample, we are able to constrain possible cosmological models. First, we
Table 11

BAO Data Vector

\begin{tabular}{lccc}
\hline \hline Parameter & Value & Survey & Source \\
\hline$D_{\mathrm{V}}(0.106)$ & $457 \pm 27 \mathrm{Mpc}$ & $6 \mathrm{dF}$ & Beutler et al. (2011) \\
$r_{\mathrm{s}} / D_{\mathrm{V}}(0.20)$ & $0.1905 \pm 0.0061$ & SDSS & Percival et al. (2010) \\
$A(0.44)$ & $0.474 \pm 0.034$ & WiggleZ & Blake et al. (2011) \\
$A(0.60)$ & $0.442 \pm 0.020$ & WiggleZ & Blake et al. (2011) \\
$A(0.73)$ & $0.424 \pm 0.021$ & WiggleZ & Blake et al. (2011) \\
$D_{\mathrm{V}}(0.35) / r_{\mathrm{s}}$ & $8.88 \pm 0.17$ & SDSS $(\mathrm{R})$ & Padmanabhan et al. (2012) \\
$D_{\mathrm{V}}(0.57) / r_{\mathrm{S}}$ & $13.67 \pm 0.22$ & BOSS & Anderson et al. (2012) \\
\hline
\end{tabular}

Notes. BAO data vector from the different surveys used for the cosmological analysis. Markov Chains for these constraints are taken from the Planck data release (Planck Collaboration et al. 2013).

Table 12

Constraints on $\Omega_{\mathrm{M}}$ and $w$

\begin{tabular}{lccccc}
\hline \hline & \multicolumn{2}{c}{ Stat Only } & & \multicolumn{2}{c}{ Stat+Sys } \\
\cline { 2 - 3 } \cline { 5 - 6 } & $\Omega_{\mathrm{M}}$ & $\Omega_{\Lambda}$ & & $\Omega_{\mathrm{M}}$ & $\Omega_{\Lambda}$ \\
\hline PS1-lz only (flat universe) & $0.242_{-0.041}^{+0.039}$ & $0.758_{-0.041}^{+0.039}$ & & $0.226_{-0.061}^{+0.057}$ & $0.774_{-0.061}^{+0.057}$ \\
PS1-1z+PL+BAO+H$+H_{0}$ & $0.274_{-0.015}^{+0.018}$ & $0.722_{-0.014}^{+0.014}$ & & $0.308_{-0.030}^{+0.033}$ & $0.693_{-0.025}^{+0.024}$ \\
\hline
\end{tabular}

Notes. Constraints on $\Omega_{\mathrm{M}}$ and $\Omega_{\Lambda}$ assuming a $\Lambda$ CDM model $(w=-1)$ with statistical only and systematic + statistical uncertainties. For the PS1-lz only sample, the constraints on $\Omega_{\mathrm{M}}$ are not significant, we therefore present the constraints assuming flatness.

Table 13

Constraints on $\Omega_{\mathrm{M}}$ and $w$

\begin{tabular}{lcclcc}
\hline \hline & \multicolumn{2}{c}{ Stat Only } & & \multicolumn{2}{c}{ Stat+Sys } \\
\cline { 2 - 3 } \cline { 5 - 6 } & $\Omega_{\mathrm{M}}$ & $w$ & & $\Omega_{\mathrm{M}}$ & $w$ \\
\hline PS1-lz only & $0.223_{-0.221}^{+0.209}$ & $-1.010_{-0.206}^{+0.360}$ & & $0.256_{-0.174}^{+0.201}$ & $-1.120_{-0.357}^{+0.450}$ \\
PS1-lz+PL+BAO+ $H_{0}$ & $0.284_{-0.010}^{+0.010}$ & $-1.131_{-0.049}^{+0.049}$ & & $0.280_{-0.012}^{+0.013}$ & $-1.166_{-0.069}^{+0.072}$ \\
\hline
\end{tabular}

Notes. Constraints on $\Omega_{\mathrm{M}}$ and $w$ assuming a flat universe with statistical only and systematic + statistical uncertainties.

assume a $\Lambda \mathrm{CDM}$ model $(w=-1)$ and measure constraints on $\Omega_{\mathrm{M}}$ and $\Omega_{\Lambda}$. Using only the PS $1+\mathrm{lz}$ sample and assuming flatness, we find $\Omega_{\mathrm{M}}=0.226_{-0.061}^{+0.057}$ and $\Omega_{\Lambda}=0.774_{-0.061}^{+0.057}$ including systematic uncertainties. We present confidence contours for these parameters in the left panel of Figure 15. The evidence for dark energy when not assuming a flat universe, from the $\mathrm{SN}$-only sample, is $99.999 \%$ when including all systematic uncertainties. We also combine the SN constraints with BAO, $\mathrm{CMB}$, and $H_{0}$ constraints to find values of $\Omega_{\mathrm{M}}=0.308_{-0.030}^{+0.033}$ and $\Omega_{\Lambda}=0.693_{-0.025}^{+0.024}$ (see left panel of Figure 16). We summarize our constraints for $\Omega_{\mathrm{M}}$ and $\Omega_{\Lambda}$ under these different assumptions in Table 12.

Relaxing the assumption of a cosmological constant, we can attempt to measure $w$, the dark energy equation-of-state parameter. For these $w C D M$ models, we assume a flat universe $\left(\Omega_{k}=0\right)$. With only the PS $1+\mathrm{lz}$ SN sample, we measure $w=$ $-1.120_{-0.206}^{+0.360}(\text { Stat })_{-0.291}^{+0.269}$ (Sys) (see right panel of Figure 15). We also combine the SN constraints with $\mathrm{CMB}, \mathrm{BAO}$, and $H_{0}$ constraints to determine $\Omega_{\mathrm{M}}=0.280_{-0.012}^{+0.013}$ and $w=$ $-1.166_{-0.069}^{+0.072}$ (see right panel of Figure 16). The first number represents the mean, and the uncertainties represent the distance between the mean and the $1 \sigma$ limits. This formalism is similar to what Planck Collaboration et al. (2013) uses in its Markov Chain release.

In Tables 13 and 14, we compare how the different cosmological probes impact the constraints on $\Omega_{\mathrm{M}}$ and $w$. As shown 

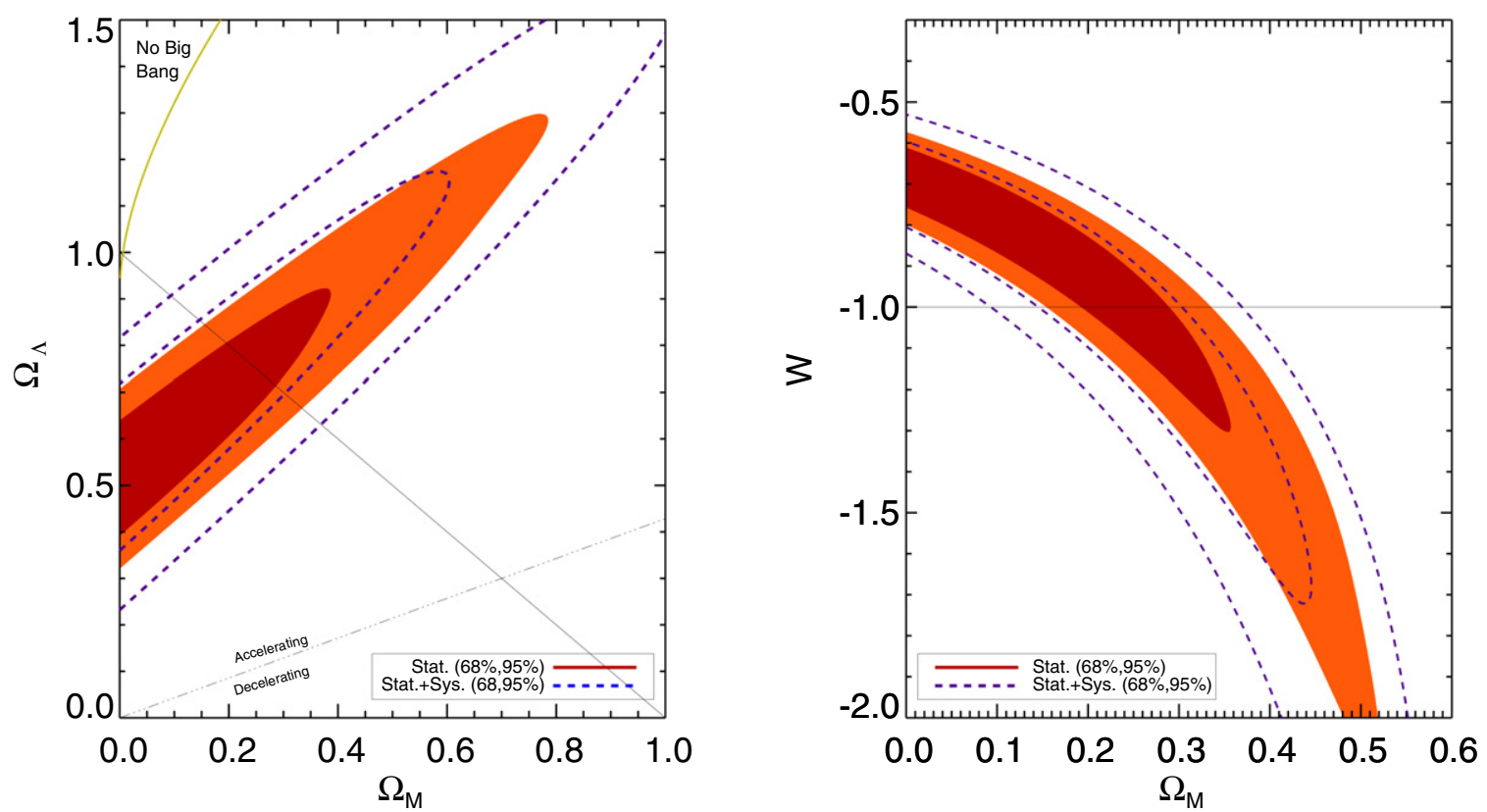

Figure 15. $1 \sigma$ and $2 \sigma$ cosmological constraints using the PS1-lz SNe sample only. The statistical constraints as well as when statistical and systematic uncertainties are combined are shown with the solid red and dashed blue lines, respectively. Left: constraints on $\Omega_{\mathrm{M}}$ and $\Omega_{\Lambda}$ assuming a cosmological constant $(w=-1)$. Right: constraints on $\Omega_{\mathrm{M}}$ and $w$ assuming a constant dark energy equation of state and flatness.

(A color version of this figure is available in the online journal.)
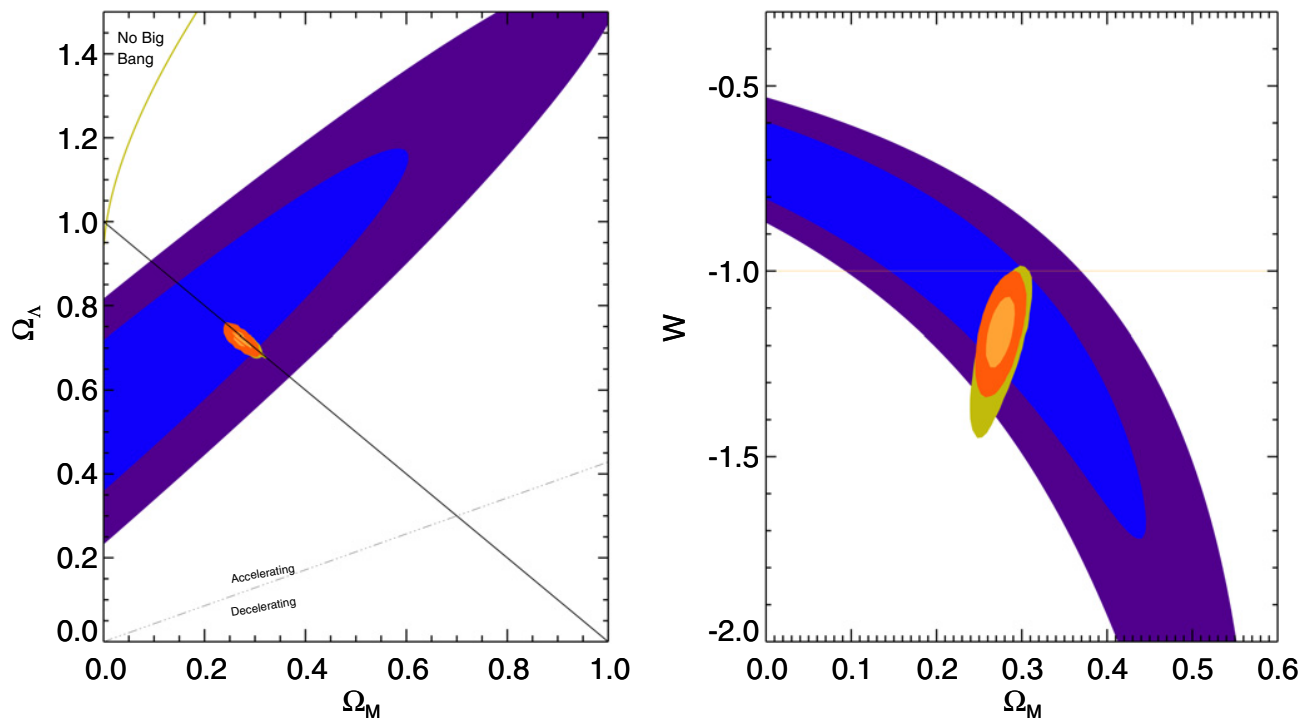

Figure 16. Cosmological constraints of $68 \%$ and $95 \%$ using PS1-1z, Planck, BAO and $H_{0}$ measurements. Here the statistical and systematic uncertainties are propagated. Left: constraints on $\Omega_{\mathrm{M}}$ and $\Omega_{\Lambda}$ assuming a cosmological constant $(w=-1)$. Right: constraints on $\Omega_{\mathrm{M}}$ and $w$ assuming a constant dark energy equation of state and flatness.

(A color version of this figure is available in the online journal.)

Table 14

Cosmological Parameter Constraints Using Different Cosmological Probe Combinations

\begin{tabular}{|c|c|c|c|c|c|c|}
\hline \multirow[t]{2}{*}{ Sample } & \multicolumn{2}{|c|}{ Without PS1-lz } & \multicolumn{2}{|c|}{ With PS1-lz (Stat. Only) } & \multicolumn{2}{|c|}{ With PS1-lz (Sys. and Stat.) } \\
\hline & $\Omega_{\mathrm{M}}$ & $w$ & $\Omega_{\mathrm{M}}$ & $w$ & $\Omega_{\mathrm{M}}$ & $w$ \\
\hline$\overline{\mathrm{PL}}$ & $0.218_{-0.079}^{+0.023}$ & $-1.485_{-0.426}^{+0.253}$ & $0.289_{-0.019}^{+0.015}$ & $-1.102_{-0.061}^{+0.058}$ & $0.281_{-0.022}^{+0.018}$ & $-1.136_{-0.079}^{+0.077}$ \\
\hline $\mathrm{PL}+\mathrm{BAO}$ & $0.287_{-0.020}^{+0.021}$ & $-1.133_{-0.104}^{+0.138}$ & $0.291_{-0.012}^{+0.010}$ & $-1.102_{-0.058}^{+0.055}$ & $0.288_{-0.014}^{+0.014}$ & $-1.124_{-0.066}^{+0.077}$ \\
\hline $\mathrm{PL}+H_{0}$ & $0.258_{-0.021}^{+0.016}$ & $-1.240_{-0.093}^{+0.095}$ & $0.277_{-0.015}^{+0.011}$ & $-1.131_{-0.052}^{+0.044}$ & $0.269_{-0.015}^{+0.016}$ & $-1.174_{-0.059}^{+0.064}$ \\
\hline $\mathrm{PL}+\mathrm{BAO}+H_{0}$ & $0.275_{-0.014}^{+0.014}$ & $-1.205_{-0.087}^{+0.102}$ & $0.284_{-0.010}^{+0.010}$ & $-1.131_{-0.049}^{+0.049}$ & $0.280_{-0.012}^{+0.013}$ & $-1.166_{-0.069}^{+0.072}$ \\
\hline
\end{tabular}

Notes. Comparison of the $\Omega_{\mathrm{M}}$ and $w$ constraints using different variations of external constraints of Planck (Planck Collaboration et al. 2013), BAO, and $H_{0}$ (Riess et al. 2011). 
in Figure 16, the constraints of $\mathrm{PL}+\mathrm{BAO}+H_{0}$ is within the $2 \sigma$ of our SN-only constraints. Using the Planck measurements alone leads to a very low value of $w=-1.485$, albeit still within $2 \sigma$ of -1 due to its uncertainties of 0.253 . Planck Collaboration et al. (2013) claim that there is significant tension between their measurements and measurements of $H_{0}$ and/or SNe (SNLS3), but not with the BAO measurements. We show in Table 14 that $\mathrm{BAO}, H_{0}$, and $\mathrm{SNe}$ measurements all have a similar pull toward a cosmological constant when combined with Planck. When combining our PS1+lz measurements with Planck, BAOs, and $H_{0}$, we find $w=-1.166_{-0.069}^{+0.072}$ inconsistent with -1 at the $2.3 \sigma$ level. We note that this is very similar to the $2.6 \sigma$ inconsistency found when substituting SNLS3 for PS1+lz (see Table 18.27 in Planck Collaboration et al. 2013). It is important to point out that a tension with a cosmological constant is greatest when $H_{0}$ and Planck constraints only are used. S14 analyzes the tension between SNe and the CMB using WMAP (Hinshaw et al. 2013) instead of Planck, and finds the tension to be smaller.

\section{DISCUSSION}

Using the first $1.5 \mathrm{yr}$ of the PS1 MDF survey, we have discovered thousands of transients, 146 of which we have spectroscopically confirmed to be $\mathrm{SNe}$ Ia. Combining novel calibration techniques with a well-tested photometric data reduction pipeline, we have obtained precise photometry for these $\mathrm{SNe}$. We estimate that the photometric uncertainty is $1.2 \%$, excluding the uncertainty in the HST Calspec definition of the AB system. Using the SALT2 light curve fitter, we have measured distances to a carefully selected sample of $113 \mathrm{SNe}$ Ia. After correcting for biases related to detection and spectroscopic follow-up efficiency, we used these $\mathrm{SNe}$ to constrain cosmological parameters.

\subsection{Comparison to Previous Work}

For our cosmological analysis, we used very recent constraints coming from BAO and CMB experiments. Previous $\mathrm{SN}$ analyses did not have access to those data, and thus a direct comparison is more difficult. Similarly, our low-redshift sample is larger than previous compilations, again, complicating any comparison. Nonetheless, we report previous results in an attempt to place the current PS1 analysis in context.

The ESSENCE survey, using 60 high-redshift SNe Ia, 45 lowredshift $\mathrm{SNe}$ Ia, and the initial SDSS BAO results (Eisenstein et al. 2005), found $w=-1.05$ with statistical and systematic uncertainties on the order of $13 \%$ (Wood-Vasey et al. 2007). The SDSS-II SN survey, using 103 high-redshift SNe Ia, 33 lowredshift $\mathrm{SNe}$ Ia, WMAP5, and the initial SDSS BAO results, found $w=-0.92 \pm 0.13$ (stat) (Kessler et al. 2009a). From combinations with SNLS and HST SNe (resulting in 288 total SNe Ia), their constraints decreased to $w=-0.96 \pm 0.06$ (stat) \pm 0.12 (sys). The 3 yr SNLS analysis used a combined sample of 242 high-redshift SNe Ia from SNLS, 93 SDSS, 14 HST, and 132 low-redshift SNe Ia (a total of $472 \mathrm{SNe}$ ), and when combined with the initial SDSS BAO results, WMAP5, and the $H_{0}$ constraint, they find $w=-1.061$ with both statistical and systematic uncertainties on the order of 5\% (Conley et al. 2011; Sullivan et al. 2011). The PS1 cosmological constraints are derived using the most recent external constraints from Planck, the Wiggle- $Z$ BAO results, and the $H_{0}$ constraint. Combining these external constraints with 113 high-redshift PS1 SNe Ia and 222 light curves from 197 low-redshift SNe Ia, we find $w=-1.166_{-0.069}^{+0.072}$, inconsistent with -1 at the $2.3 \sigma$ level. It is currently still unclear whether the tension with flat $\Lambda \mathrm{CDM}$ is a feature of the model or a combination of chance and systematic errors.

All the other SN Ia surveys of the past decade constrain $w$ to the cosmological constant value of -1 within $1 \sigma$. An outside observer might expect one-third of the results to lie outside this bound, and a confirmation bias could be in effect (Croft \& Dailey 2011). Superficially these may appear to be independent tests, providing mounting evidence for a $w=-1$ universe. However, there is significant overlap in the low-redshift SN data used in all analyses, and we should thus expect some degree of correlation in the results. Furthermore, most SN surveys also share the same calibration sources.

\subsection{Future Improvements to Photometric Calibration}

Our systematic uncertainties are dominated by the photometric calibration. Although our calibration effort is sufficient for our current analysis and comparable to other SN surveys, there are additional areas in which significant improvements can be made. Here, we identify areas for future improvement.

Since photometric calibration is currently the largest component of the systematic error budget, it should be the primary focus for future efforts. In order to reduce the systematic uncertainty of the calibration to the level of other systematic uncertainties, photometric accuracy must be significantly better than $1 \%$. For the current PS1 photometric system, all indications are that we have an accuracy of $1.2 \%$ before accounting for the HST Calspec uncertainty in the AB magnitude system. Over the coming years, we expect that the NIST-based tunable laser system will continue to improve, resulting in decreased ghosting and a more uniform illumination of the flat-field screen. The PS1 photometry is also still improving through changes in reduction software and the acquisition of new data (which will continue until early in 2014 for the MDF survey).

Recently, the SNLS and SDSS teams have undertaken an effort to precisely calibrate their photometric systems onto roughly a single system. They have reached a $0.5 \%$ consistency between their photometric systems, using methods similar to those employed in the PS1 analysis. Specifically, they used observations of the HST Calspec standard stars in both systems to provide consistency. An attempt of this analysis is done by S14, which finds discrepancies of up to $2 \%$ between the PS1 and SDSS AB systems. The large overlap between the SDSS photometric footprint and the PS $13 \pi$ survey has already been used in an übercalibration of the PS1 system (Schlafly et al. 2012). This type of analysis can provide further improvement. In addition, the ongoing HST program GO-12967 measures fluxes of DA white dwarf stars, one of which resides within a PS1 MDF. This will allow an in situ direct comparison of white dwarf colors with models that incorporate $\log (g)$ and temperature from ground-based spectroscopy.

We believe that we can also improve our estimation of flux uncertainties. Specifically, we know that there is a tail to the reduced $\chi^{2}$ distribution shown in Figure 7 to large $\chi^{2}$. We have shown that these outliers are SNe close to bright objects (usually a host galaxy nucleus), resulting in an underestimate of the uncertainty. We have performed preliminary tests that indicate that modeling the uncertainties with an additional term proportional to the distance to the nearest bright static object will remove this tail and provide a better estimate of the uncertainty. Only a small fraction of SNe are significantly affected by this bias, so it does not affect our current results, but should become more important as we reduce other systematic uncertainties. 


\subsection{Improvements to Cosmological Constraints}

The data presented here comprises the first $1.5 \mathrm{yr}$ of PS1. As is common with large projects, the data quality and cadence at the beginning of the survey were not as good as those achieved later in the survey. We expect that typical light curve coverage and $\mathrm{S} / \mathrm{N}$ for the full PS1 sample will be better than in the current sample. At the end of the survey, the total exposure time for the MDF survey will exceed the exposure time of the current sample by nearly a factor of three. In addition, our spectroscopic followup efficiency has increased; we expect a final spectroscopic sample will consist of $\gtrsim 400 \mathrm{SN}$ Ia. Thus we can expect that our current statistical uncertainties will improve by a factor of $\sim$ two, comparable to the best statistical uncertainties currently reported.

Along with the large spectroscopic sample, we will observe several times as many SNe Ia without spectra. We can generate a large photometrically classified sample of SNe Ia with relatively small contamination from other SN classes (Sako et al. 2011). The SDSS-II SN survey produced such a sample consisting of 752 photometrically classified SNe Ia and produced cosmological constraints with this sample (Campbell et al. 2013). Combining distance estimates of these $\mathrm{SNe}$ (and no other data sets, i.e., no low-redshift $\mathrm{SNe}$ ) with constraints on $H_{0}$, data from WMAP, and large-scale structure measurements, they find constraints on $w$ with statistical uncertainties of $10 \%$.

The advantage of this method is that the sample size is not limited by the availability of spectroscopic follow-up resources, which has not scaled with the number of SNe discovered. PS1 has discovered $\gtrsim 4500$ transients with SN-like light curves. This number will likely increase to $\gtrsim 6000$ by the end of the PS1 survey. In magnitude-limited surveys like PS1, 79\% of all SNe are $\mathrm{SNe} \mathrm{Ia}$ (Li et al. 2011), thus with a classification efficiency of $70 \%$, we can expect $\sim 3300$ photometrically classified SNe Ia from the PS1 survey.

The photometric classification of a transient is significantly improved with a host-galaxy spectroscopic redshift, which can be obtained after the SN has faded. Follow-up observations at the end of the survey with a large FOV multi-object spectrograph such as Hectospec on the MMT should provide redshifts for the vast majority of candidate SNe Ia (D. Jones et al. 2014, in preparation).

With this large set of new SNe Ia, more work can be done to identify a "third parameter" that correlates with luminosity independent of light curve shape and color. This would be particularly important if there is some redshift-dependent evolution of the SNe Ia properties. Candidates are, for example, ejecta velocity (Wang et al. 2009; Foley \& Kasen 2011; Foley et al. 2011), other spectral features (Foley et al. 2008; Bailey et al. 2009; Blondin et al. 2011; Silverman et al. 2012b; Foley \& Kirshner 2013), and host galaxy properties (Kelly et al. 2010; Lampeitl et al. 2010; Sullivan et al. 2010; Hayden et al. 2013).

We are also conducting a large (100 orbit) HST program, RAISIN (GO-13046; PI: Kirshner) to obtain rest-frame NIR photometry of PS1 SNe Ia $(0.2<z<0.4)$. Although calibration is currently the largest systematic uncertainty in our analysis, the largest astrophysical systematic uncertainty comes from the treatment of intrinsic SN colors and dust reddening, which directly affects distance measurements. When combining the HST and PS1 photometry, the large wavelength range (from UV to NIR) provides additional constraints on the reddening law, which should significantly improve our understanding of systematics related to dust extinction, reddening, and SN Ia colors. SNe Ia are more nearly standard candles when observed in rest-frame near-infrared bands and extinction is significantly lower at these wavelengths (Krisciunas et al. 2004; Wood-Vasey et al. 2008; Mandel et al. 2009; Folatelli et al. 2010; BaroneNugent et al. 2012; Kattner et al. 2012). The systematic errors that result from light curve shape corrections and inferences about extinction from color are distinctly smaller when using these bands (Mandel et al. 2011).

\section{CONCLUSION}

We have presented the light curves and analysis of 146 spectroscopically confirmed SNe Ia from the first $1.5 \mathrm{yr}$ of the PS1 Medium Deep Survey. We have described the SN discovery and spectroscopic followup of the survey. We analyze the relative and absolute photometric calibration in the PS1 natural system using on-site measurements of the instrument response function, spectro-photometric standard star observations, and übercalibration, with an emphasis on any systematic biases introduced. We find that the systematic uncertainties in the photometric calibration are currently $1.2 \%$ without accounting for the uncertainty in the HST Calspec definition of the AB system.

From the sample of $146 \mathrm{SNe}$ Ia in the redshift range $0.03<z<0.65,113$ passed the various quality cuts in our cosmological analysis. The spectral information and SALT2 light curve fit parameters of each $\mathrm{SN}$ are presented so that this sample can be used in joint analyses with other SN samples. When combining the PS1 sample with low- $z$ SNe Ia (113 PS1 SNe Ia +222 light curves from 197 low- $z$ SNe Ia), we find $w=-1.120_{-0.357}^{+0.450}$ assuming a flat universe. A universe devoid of dark energy is rejected at the at $99.999 \%$ level with the SN sample alone including all identified systematics. When combined with external constraints (BAOs, CMB, and $H_{0}$ ), our cosmological analysis yields $\Omega_{\mathrm{M}}=0.280_{-0.012}^{+0.013}$ and $w=$ $-1.166_{-0.069}^{+0.072}$ including all identified systematics, consistent with a cosmological constant. This is in agreement with the results from previous SN Ia surveys like SNLS and SDSS. We show the pull on the recovered cosmological parameters from the $\mathrm{SN}$ measurements. Compared to when $\mathrm{CMB}, \mathrm{BAO}$, and $H_{0}$ measurements are used to constrain $w$, including the SN measurements reduces the total uncertainty in $w$ by $\sim 40 \%$.

Further analysis of the systematic errors of this sample is presented in the companion paper by S14. In the future analysis of the full four yr PS1 sample, we can reduce the dominant systematic uncertainty in absolute photometric calibration with more PS1 observations, additional Calspec spectrophotometric standards, and improved reduction and analysis.

This paper and the companion paper by S14 represent the first in a series of cosmological analysis using the PS 1 sample. It is necessary to accurately measure SN photometry and quantify systematic uncertainties with the spectroscopically confirmed sample before doing so for the much larger photometric sample. The photometric sample is currently $>5$ times bigger than any published spectroscopic sample of SN Ia and will represent a significant step forward for constraining the nature of dark energy.

The Pan-STARRS1 (PS1) Surveys have been made possible through contributions of the Institute for Astronomy, the University of Hawaii, the Pan-STARRS Project Office, the Max-Planck Society and its participating institutes, the Max Planck Institute for Astronomy, Heidelberg and the Max Planck 
Institute for Extraterrestrial Physics, Garching, The Johns Hopkins University, Durham University, the University of Edinburgh, Queen's University Belfast, the Harvard-Smithsonian Center for Astrophysics, the Las Cumbres Observatory Global Telescope Network Incorporated, the National Central University of Taiwan, the Space Telescope Science Institute, the National Aeronautics and Space Administration under grant No. NNX08AR22G issued through the Planetary Science Division of the NASA Science Mission Directorate, the National Science Foundation under grant no. AST-1238877, the University of Maryland, and Eotvos Lorand University (ELTE). Some observations reported here were obtained at the MMT Observatory, a joint facility of the Smithsonian Institution and the University of Arizona. This paper is based on observations obtained at the Gemini Observatory, which is operated by the Association of Universities for Research in Astronomy, Inc., under a cooperative agreement with the NSF on behalf of the Gemini partnership: the National Science Foundation (United States), the National Research Council (Canada), CONICYT (Chile), the Australian Research Council (Australia), Ministério da Ciência, Tecnologia e Inovação (Brazil) and Ministerio de Ciencia, Tecnología e Innovación Productiva (Argentina). This paper includes data gathered with the $6.5 \mathrm{~m}$ Magellan Telescopes located at Las Campanas Observatory, Chile and observations obtained with the Apache Point Observatory $3.5 \mathrm{~m}$ telescope, which is owned and operated by the Astrophysical Research Consortium. C.W.S. and G.N. thank the DOE Office of Science for their support under grant ER41843. Partial support for this work was provided by National Science Foundation grant AST-1009749. The ESSENCE/SuperMACHO data reduction pipeline photpipe was developed with support from National Science Foundation grant AST-0507574, and HST programs GO-10583 and GO-10903. R.P.K.'s supernova research is supported in part by NSF grant AST-1211196 and HST program GO-13046. Some of the computations in this paper were run on the Odyssey cluster supported by the FAS Science Division Research Computing Group at Harvard University. This research has made use of the CfA Supernova Archive, which is funded in part by the National Science Foundation through grant AST 0907903. This research has made use of NASA's Astrophysics Data System.

Facilities: PS1 (GPC1), Gemini:South (GMOS), Gemini:Gillett (GMOS), MMT (Blue Channel spectrograph, Hectospec), Magellan:Baade (IMACS), Magellan:Clay (LDSS3).

\section{APPENDIX A}

\section{ASTROMETRIC ACCURACY}

Accurate astrometry is critical for the discovery and classification of $\mathrm{SNe}$ from a sequence of observations. In addition, forced photometry requires accurate positions as input (see Section 5.3.2), therefore it is crucial that the astrometry not exhibit any systematic biases. In this appendix, we assess the accuracy of our PS1 astrometry, and quantify its impact on our overall systematic error budget.

Starting with the reported WCS from the PS1 IPP, we finetune the linear terms that define the translation and rotation in $10^{\prime} \times 10^{\prime}$ cutouts centered on the $\mathrm{SN}$. The astrometric uncertainty $\sigma_{a}$ can be described by a systematic floor $\sigma_{a 1}$ and a Poissonian term $\sigma_{a 2}$ that scales with the FWHM and S/N:

$$
\sigma_{a}^{2}=\sigma_{a 1}^{2}+\sigma_{a 2}^{2}\left(\frac{\mathrm{FWHM}}{\mathrm{S} / \mathrm{N}}\right)^{2}
$$

The value of $\sigma_{a 1}$ is sensitive to the accuracy of the distortion terms, and the number of stars used to determine the WCS solution. If the PSF is undersampled, $\sigma_{a 1}$ is limited by the pixelation and can be as low as 0.05 pixels. For a given telescope/detector system, $\sigma_{a 1}$ and $\sigma_{a 2}$ are global parameters, and our goal is to determine them for the PS1 system. This allows us then to determine the astrometric uncertainty for a given detection depending on its FWHM and S/N.

We compare the detections from a nightly stack with the detections from a deep stack. The uncertainty $\sigma_{\Delta}$ in the difference of position between the detections in the nightly and the deep stack depends then on the S/N and FWHM of both, the deep and the nightly stacks. However, it can safely be assumed that $\mathrm{S} / \mathrm{N}_{\text {deep }} \gg \mathrm{S} / \mathrm{N}_{\text {nightly }}$, and also that its FWHM is similar or better. Therefore, the $\sigma_{a 2}$ term from the deep stack is negligible, and we can write

$$
\begin{gathered}
\sigma_{\Delta}^{2}=\sigma_{a, \text { nightly }}^{2}+\sigma_{a, \text { deep }}^{2} \\
=2 \sigma_{a 1}^{2}+\sigma_{a 2}^{2}\left(\frac{\mathrm{FWHM}_{\text {nightly }}}{\mathrm{S} / \mathrm{N}_{\text {nightly }}}\right)^{2}+\sigma_{a 2}^{2}\left(\frac{\mathrm{FWHM}_{\text {deep }}}{\mathrm{S} / \mathrm{N}_{\text {deep }}}\right)^{2} \\
\approx 2 \sigma_{a 1}^{2}+\sigma_{a 2}^{2}\left(\frac{\mathrm{FWHM}_{\text {nightly }}}{\mathrm{S} / \mathrm{N}_{\text {nightly }}}\right)^{2} .
\end{gathered}
$$

Figure 17 shows the pixel position difference $\Delta X$ (R.A. on the sky) and $\Delta Y$ (Decl. on the sky) between the nightly and deep stacks for the stars in our photometric standard star catalog (T12b) of 100 randomly selected images in the $g_{\mathrm{P} 1}$ band with a plate scale of 0.2 arcsec pixel $^{-1}$. We have done a similar fit for the other filters as well. The mean and standard deviation of the mean are shown with red symbols in appropriately spaced $(\mathrm{FWHM} / \mathrm{S} / \mathrm{N})^{2}$ bins. We fit a straight line to $\sigma_{\text {Delta }}^{2}$ versus $(\mathrm{FWHM} / \mathrm{S} / \mathrm{N})^{2}$ for all filters to determine $\sigma_{a 1}$ and $\sigma_{a 2}$ from the slope and intercept of the fit. The fit is very good, and we find

$$
\begin{gathered}
\sigma_{a 1, X}=0.135 \pm 0.010 \text { pixels }=27 \pm 2 \text { mas } \\
\sigma_{a 1, Y}=0.105 \pm 0.015 \text { pixels }=21 \pm 3 \text { mas }
\end{gathered}
$$

$$
\sigma_{a 2, X}=1.379 \pm 0.012
$$

$$
\sigma_{a 2, Y}=1.348 \pm 0.011
$$

We find no differences in a given direction for the different filters, however we note that $\sigma_{a 1}$ does differ at the $2 \sigma$ level between the $X$ and $Y$ direction. This discrepancy might be due to the Koppenhoefer effect, in which the positions of objects are biased in the $X$ direction by an amount that increases for brighter objects. This effect was present in half of the GPC1 chips from the start of the mission until 2011 May, at which point modifications to the camera voltages successfully removed the bias. For the affected chips, the maximum displacement (for objects approaching saturation) is in the range of $0.1-0.15$ arcsec (up to $\sim 0.6$ pixels), depending on the device. Objects with fewer than $\sim 25,000$ counts are essentially unaffected. As a result, the generally faint objects of interest in this article are not directly affected. The main effect comes from the astrometric 

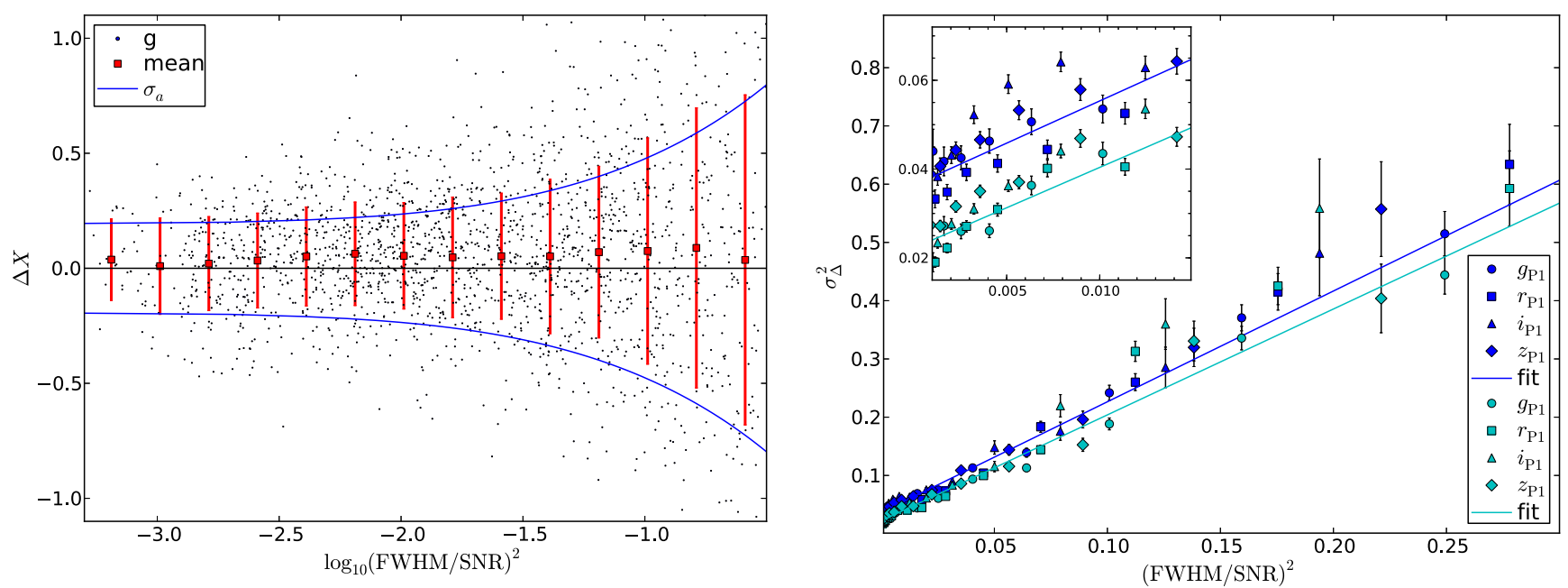

Figure 17. Left panel: the position difference $\Delta X$ in pixels of nightly and deep stacks for stars from 100 randomly selected $g_{\mathrm{P} 1}$ band images (black dots) vs. $\log _{10}(\mathrm{FWHM} /(\mathrm{S} / \mathrm{N}))^{2}$. The red circles and its error bars indicate the average $\Delta X$ in appropriate $(\mathrm{FWHM} /(\mathrm{S} / \mathrm{N}))^{2}$ bins and its standard deviation $\sigma_{\Delta X}$, respectively. The blue line is the fitted $\sigma_{\Delta X}$ from the fit shown in the right panel. Right panel: the measured variance $\sigma_{\Delta X}^{2}$ and $\sigma_{\Delta Y}^{2}$ in blue and cyan symbols, respectively, for all four bands vs. $(\mathrm{FWHM} /(\mathrm{S} / \mathrm{N}))^{2}$. The straight line fits are shown with the solid lines. The inset shows that for very small $(\mathrm{FWHM} /(\mathrm{S} / \mathrm{N}))^{2}$, there is a significant difference in $X$ and $Y$ direction.

(A color version of this figure is available in the online journal.)

calibration, which uses the brighter stars, and likely accounts for the enhanced astrometric scatter in the $X$ direction (E. A. Magnier et al., in preparation).

However, we find that this effect has only an insignificant impact on our photometry ( $<1 \mathrm{mmag}$ ). We find small average differences between the positions measured in the nightly and deep stacked images on the order of 10 mas, and we demonstrate that this also has negligible impact on our forced photometry in Section 5.3.2. Conservatively, we adopt

$$
\begin{gathered}
\sigma_{a 1}=0.2 \\
\sigma_{a 2}=1.5
\end{gathered}
$$

when we use Equation (A1) to determine the astrometric uncertainty in a given detections, e.g., when we cluster detections into objects.

\section{A.1. SN Centroids}

Accurate WCS is necessary, but not sufficient to ensure unbiased forced photometry: difference image artifacts from poor host-galaxy subtraction or other background sources, can introduce additional systematic biases. We found that that a value of $\sigma_{a 1}=0.2$ adequately takes into account these additional biases. For a given SN, we use all detections to re-determine the centroid, calculating the average, weighted by the astrometric errors determined using Equation (A1).

\section{APPENDIX B}

\section{PSF MODELING}

For identifying transients with photpipe, we use a customized version of DoPHOT, which is quick, robust, and produces adequate photometry. However, PS1 has a PSF with structure that cannot accurately fit with such an analytic model. This may cause systematic biases in the photometry between faint and bright stars of up to $1 \%$. The left of Figure 18 shows an example of the difference $\Delta g=g_{\text {PS1,DoPHOT }}-g_{\text {PS1,DAOPHOT }}$ between
DoPHOT and DAOPHOT photometry. There is a clear trend from bright to faint magnitudes. DAOPHOT fits an empirical correction in addition to the Gaussian model, which significantly better fits the PSF in the image.

The right side of Figure 18 shows the DAOPHOT flux (black symbols) and PSF fit (red line) for $r=17.5$ and $r=20.8$ example stars in the upper left and right panels, respectively. In the lower panels, the residual between the flux and the PSF model (black symbols), normalized by the uncertainty, is shown for 10 randomly selected stars in the magnitude bins $17.5<r<18.5$ and $20.5<r<21.5$. The red circles indicate the average normalized residual $\Delta f_{\text {norm }}$ for appropriate radial bins, and the error bars indicate its standard deviation. The standard deviation is $\sim 1$ for large radii, but increases to values on the order of up to 1.7 for radii closer to the core. This indicates that some of the PSF structure is still not perfectly fitted for. Most important though, there is no significant bias toward higher or lower fit values for any distance to the core for both the faint and bright stars. We therefore use DAOPHOT photometry for all the analysis in transphot.

\section{APPENDIX C}

\section{EMPIRICAL ADJUSTMENT OF UNCERTAINTIES}

The propagated uncertainties are underestimates, as they do not account for the pixel-pixel covariance introduced by warping, sub-sampling, stacking, and convolution of the images. In order to empirically determine by how much the uncertainties are underestimated, we measure the flux $f_{r}$ and its uncertainty $\sigma_{r}$ at random positions in a given difference image in exactly the same way we measure the SN flux. We calculate the weighted mean $\bar{f}_{r}$ of these flux measurements. In order to guard against reduction and image artifacts, we apply a $3 \sigma$ cut to the normalized flux distribution $\left(f_{r}-\bar{f}_{r}\right) /\left(s_{r} \sigma_{r}\right)$, rather than cutting on the underestimated errors, $\sigma_{r}$, for the following reason: let us assume that all uncertainties are underestimated by the same factor $s_{r}$. If we nominally apply an $N \sigma$ cut using these underestimated uncertainties, we effectively apply an 

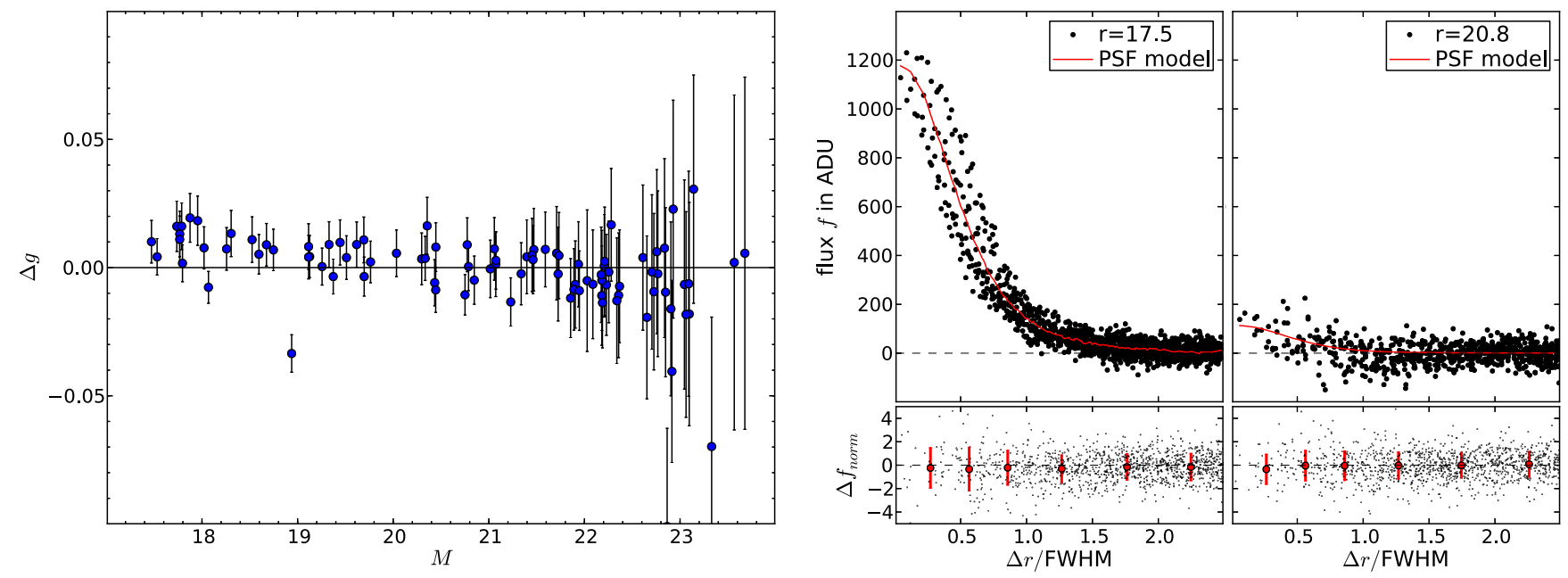

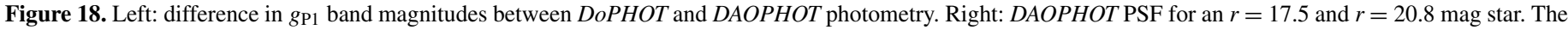

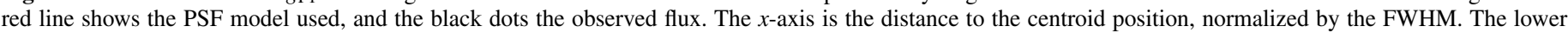

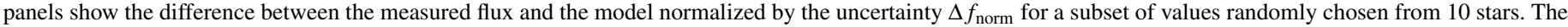
red circles indicate the average $\Delta f_{\text {norm }}$ for appropriate radial bins, and the error bars indicate the standard deviation.

(A color version of this figure is available in the online journal.)

$N / s_{r}$ cut, e.g., for a nominal $3 \sigma$ cut and $s_{r}=1.5$, the real cut-off is at $2 \sigma$. In order to avoid this, we determine the normalized flux distribution $\left(f_{r}-\bar{f}_{r}\right) / \sigma_{r}$, which has a standard deviation of $s_{r}$. The true $3 \sigma$ outliers can then be identified and removed by doing an $3 \sigma$ cut on the normalized flux distribution. Note that the standard deviation $s_{r}$ is equivalent to the square-root of the chi-square distribution

$$
s_{r}=\sqrt{\chi_{r}^{2}}=\frac{1}{N-2} \sum^{N}\left(\frac{f_{r}-\bar{f}_{r}}{\sigma_{r}}\right)^{2}
$$

We multiply all uncertainties by the factor $s_{r}$ in order to empirically correct the uncertainties. We find that it is imperative to employ this robust way of determining $s_{r}$ for the method to work correctly. The fact that the reduced chi-square of the baseline flux of the SN light curves peaks at 1.0 validates our method (see Section 5.3.4).

In addition, for a given difference image, $\bar{f}_{r}$ is an estimate of the bias in the flux measurements. The values of $\bar{f}_{r}$ are in general very small, much smaller than the typical uncertainties. Nevertheless, we adjust all fluxes by this value.

\section{APPENDIX D}

\section{CORRECTION OF SYSTEMATIC MAGNITUDE BIASES DUE TO CENTROIDING ERRORS}

We determine the position of a given $\mathrm{SN}$ as the weighted mean of all its detections. This SN centroid (R.A. 0 , Decl. ${ }_{0}$ ) has a nonnegligible astrometric uncertainty $\sigma_{\mathrm{SN} \text {,cent }}$, which introduces a bias in our recovered photometry and must be corrected. The recovered position of a detection can be different from the SN centroid for the following reasons:

1. Poisson noise, in particular positive noise peaks, in the background sky,

2. Poisson noise in the SN flux,

3. difference image artifacts,

4. the centroid accuracy of (R.A. ${ }_{0}$, Decl. $\left._{.0}\right)$, and

5. accuracy of the WCS for a given image.
The first three items in the list above introduce a Malmquist bias for regular photometry since the freedom in position will bias the fitted PSF to be centered toward the positive noise peaks. This effect is stronger for detections with low $\mathrm{S} / \mathrm{N}$. However, using forced photometry eliminates this bias (see also Section 5.3.2 and Figure 5). What is not corrected for is if the position used for forced photometry of the $\mathrm{SN}$ is offset from the true position of the $\mathrm{SN}$, either because the uncertainty in $\mathrm{SN}$ centroid or due to inaccuracies of the WCS for the given image. In this section, our goal is to characterize how the photometric bias depends on the astrometric offset, so that we can estimate the expectation value of the photometric bias for a given centroid accuracy and correct for it.

For our analysis, we use detections of PS1-10axx, a SN Ia at $z=0.027$ with a good selection of detections with both high and low S/N. As described in Section 5.2, the position (R.A.0, Decl. ${ }_{0}$ ) was determined by calculating the $3 \sigma$ clipped weighted mean of all detections. With forced photometry, (R.A. 0 , Decl. $\left.{ }_{0}\right)$ is translated into $\left(X_{0}, Y_{0}\right)$ for a given image, and the center of the PSF is forced to be at $\left(X_{0}, Y_{0}\right)$. We investigate how the photometry depends on the centroid position by measuring the photometry at position $(X, Y)=(X+\Delta X, Y+$ $\Delta Y)$. We define the change in photometry as

$$
\Delta m(\Delta X, \Delta Y)=m\left(X_{0}+\Delta X, Y_{0}+\Delta Y\right)-m\left(X_{0}, Y_{0}\right) .
$$

For simplicity, we concentrate on one dimension $(\Delta X)$, and later on apply it to two dimensions. In the right panel of Figure 19, we show $\Delta m(\Delta X)$ for detections of PS1-10axx high $\mathrm{S} / \mathrm{N}$. Without noise, $\Delta m(\Delta X)$ would be a parabola with a minimum at $\Delta X=0$, but due to a combination of the reasons listed above, the parabola is shifted. We fit these parabolas with

$$
\Delta m(\Delta X)=h\left(\Delta X-\Delta X_{\min }\right)^{2}+\Delta m_{\min },
$$

where $\Delta X_{\min }, \Delta m_{\min }$ are the coordinates of the minimum of the parabola for a given detection, and $h$ defines the width of the parabola.

If we assume that this shift in the position of the minimum is due to the error in the SN centroid, then the SN centroid used 

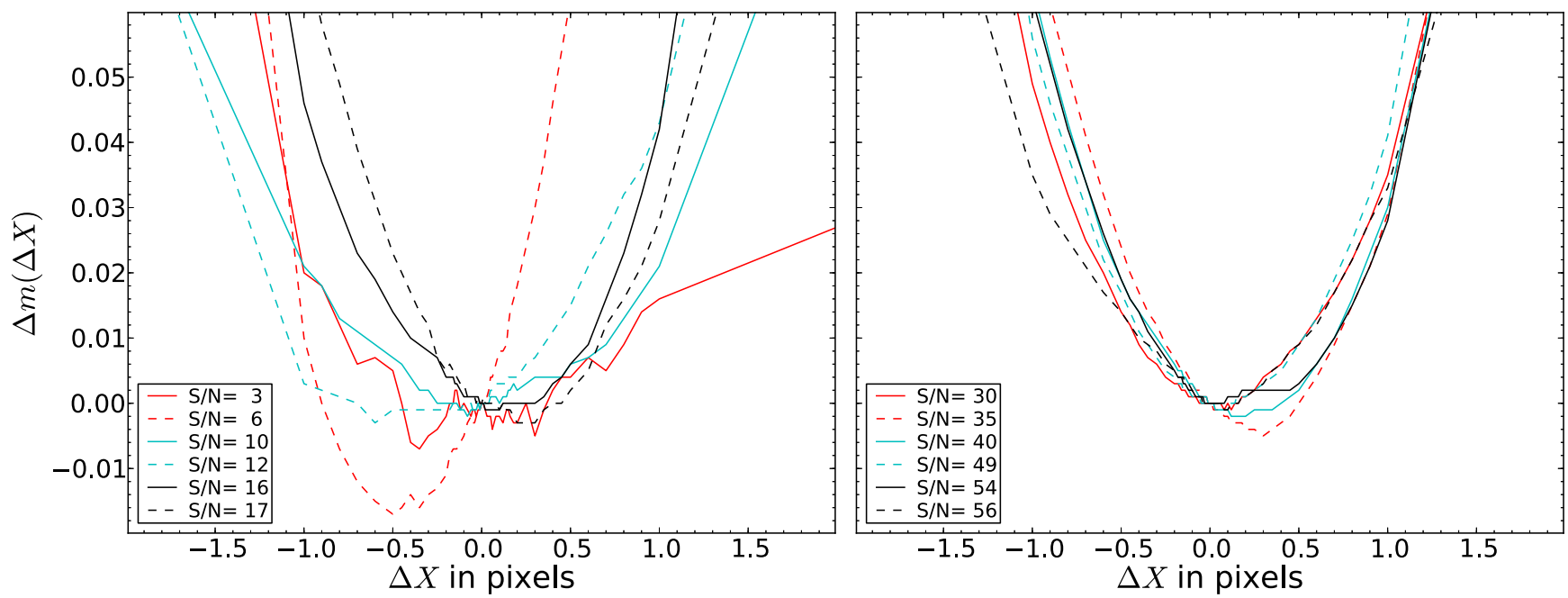

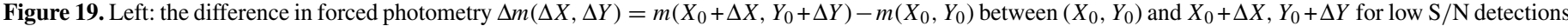
of PS1-10axx. Right: same as the left panel, but for high S/N detections of PS1-10axx.

(A color version of this figure is available in the online journal.)
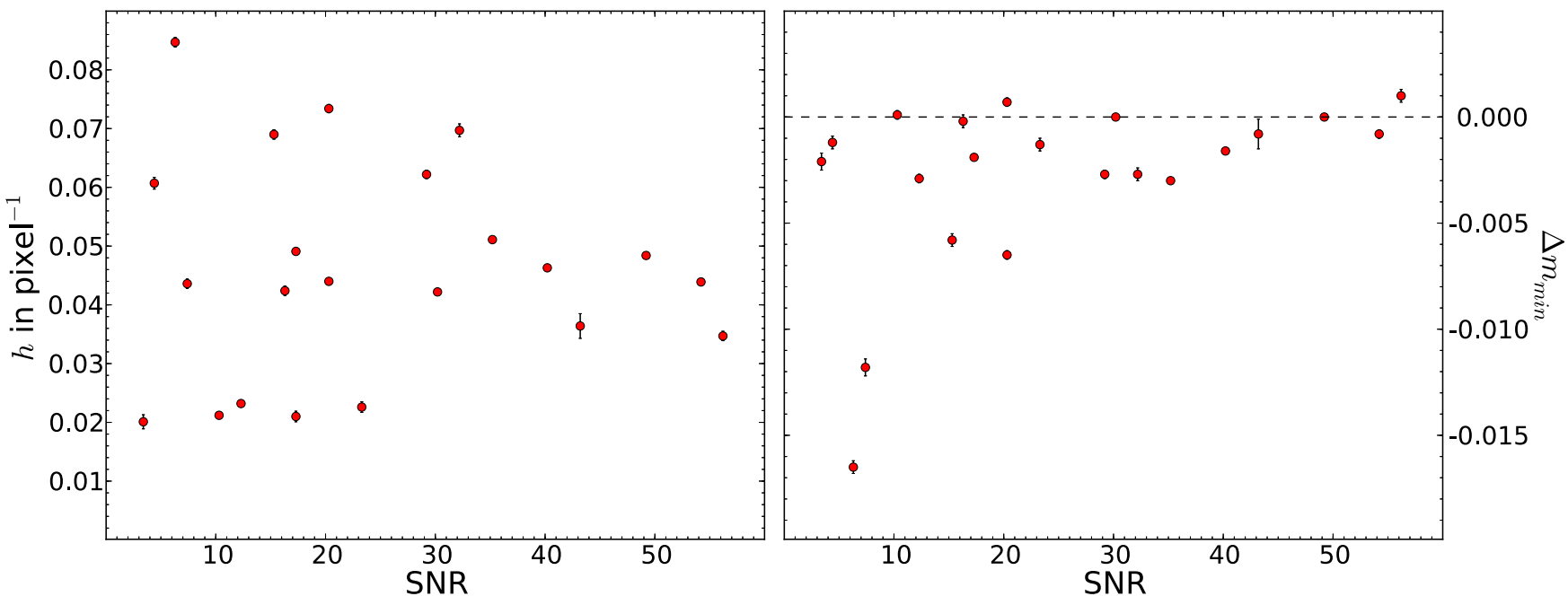

Figure 20. Left: parameter $h$ from Equation (D2) derived by fitting $\Delta m(\Delta X)$ with a parabola. Right: parameter $\Delta m_{\mathrm{min}}$ from Equation D2).

(A color version of this figure is available in the online journal.)

was off by $\Delta X_{\min }$ from the true centroid, and the photometry was biased by $\Delta m_{\min }$. Now we can turn around this argument. If we know $h$, the width of the parabola, and the centroid accuracy of a given $\mathrm{SN}$ centroid $\sigma_{\mathrm{SN}, \text { cent, } X}$, we can calculate the expectation value of the photometric bias $\Delta m_{\mathrm{SN}, \text { cent, } X}$ as

$$
\Delta m_{\mathrm{SN}, \text { cent }, X}=\int h t^{2} \operatorname{PDF}\left(\sigma_{\mathrm{SN}, \text { cent }, X}, t\right) d t,
$$

where $\operatorname{PDF}(\sigma, \mathrm{t})$ is the probability density function with sigma $\sigma$, and assuming that $h$ is constant and independent of $\mathrm{S} / \mathrm{N}$.

The assumption that the width of the parabola is constant and independent of $\mathrm{S} / \mathrm{N}$ appears to valid to the first order. For high $\mathrm{S} / \mathrm{N}$, the width of the parabola seems to be constant (see right panel of Figure 19). For low $\mathrm{S} / \mathrm{N}$ detections, however, the width of the parabola show a much larger variation (see left panel of Figure 19). Figure 20 shows the fitted $h$ and $\Delta m_{\min }$ for all detections of PS1-10axx in the left and right panels, respectively. For low $\mathrm{S} / \mathrm{N}$, the spread in $h$ significantly increases because of Poisson fluctuations in the flux and/or difference image artifacts. We therefore use the $h$ derived only from the $\mathrm{S} / \mathrm{N}>35$ detections:

$$
h=0.043 \pm 0.003 \mathrm{pixel}^{-2}
$$

with a standard deviation of 0.007 . Any effect of the spread in $h$ on $\Delta m_{\mathrm{SN} \text {, cent, } X}$ cancels out to the first order, as long the mean $h$ is independent of $\mathrm{S} / \mathrm{N}$.

For a given $\mathrm{SN}$, we estimate the uncertainty in the $\mathrm{SN}$ centroid $\sigma_{\mathrm{SN}, \text { cent }, X}$ and $\sigma_{\mathrm{SN}, \text { cent }, Y}$ when we calculate the $\mathrm{SN}$ position (R.A.0, Decl. 0 ) as the weighted mean of all the SN detections. Using the fitted $h$ and Equation (D3) we can now calculate the expectation value of the systematic bias $\Delta m_{\mathrm{SN} \text {, cent }}$ in our $\mathrm{SN}$ photometry:

$$
\Delta m_{\mathrm{SN}, \text { cent }}=\Delta m_{\mathrm{SN}, \text { cent }, X}+\Delta m_{\mathrm{SN}, \text { cent }, Y} .
$$

The left panel of Figure 21 shows $\sigma_{\mathrm{SN}, \mathrm{cent}}=$ $\sqrt{\sigma_{\mathrm{SN}, \text { cent }, X}^{2}+\sigma_{\mathrm{SN}, \text { cent }, X}^{2}}$ for all SNe versus the redshift, and the right panel shows $\Delta m_{\mathrm{SN} \text {,cent }}$. 

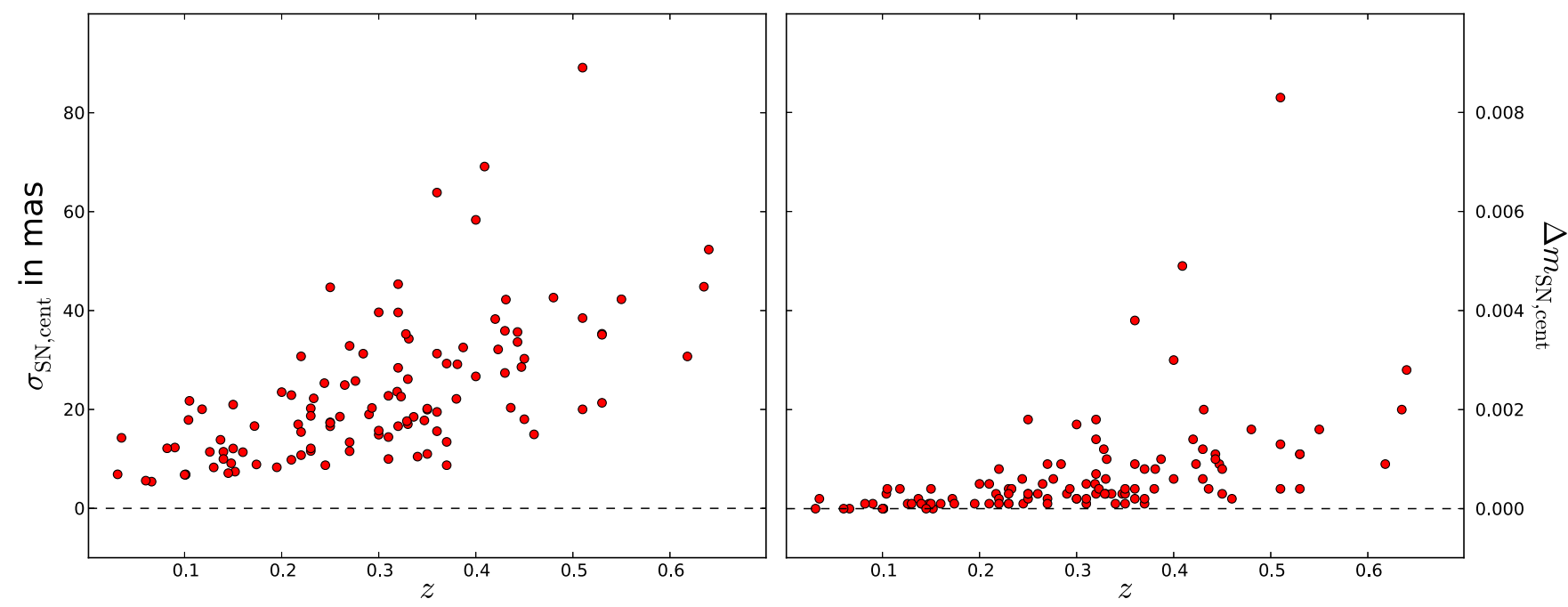

Figure 21. Left: the astrometric uncertainty in the SN centroid position determined by averaging the position of all detections vs. redshift. The uncertainty in the SN centroid increases with increasing redshift. Right: the expectation value of the photometric bias due to the centroid uncertainty calculated with Equation (D5). (A color version of this figure is available in the online journal.)

The photometric bias has a systematic trend with redshift on the order of $2 \mathrm{mmag}$, and these corrections are added to our final photometry.

\section{REFERENCES}

Anderson, L., Aubourg, E., Bailey, S., et al. 2012, MNRAS, 427, 3435 Astier, P., Guy, J., Regnault, N., et al. 2006, A\&A, 447, 31

Bailey, S., Aldering, G., Antilogus, P., et al. 2009, A\&A, 500, L17 Barone-Nugent, R. L., Lidman, C., Wyithe, J. S. B., et al. 2012, MNRAS, 425,1007

Berger, E., Chornock, R., Lunnan, R., et al. 2012, ApJL, 755, L29

Berger, E., Leibler, C. N., Chornock, R., et al. 2013, ApJ, 779, 18

Betoule, M., Marriner, J., Regnault, N., et al. 2013, A\&A, 552, A124

Beutler, F., Blake, C., Colless, M., et al. 2011, MNRAS, 416, 3017

Blake, C., Kazin, E. A., Beutler, F., et al. 2011, MNRAS, 418, 1707

Blondin, S., Mandel, K. S., \& Kirshner, R. P. 2011, A\&A, 526, A81

Blondin, S., \& Tonry, J. L. 2007, ApJ, 666, 1024

Bohlin, R. C. 1996, AJ, 111, 1743

Bohlin, R. C. 2007, in ASP Conf. Ser. 364, The Future of Photometric, Spectrophotometric and Polarimetric Standardization, ed. C. Sterken (San Francisco, CA: ASP), 315

Bohlin, R. C., Dickinson, M. E., \& Calzetti, D. 2001, AJ, 122, 2118

Bohlin, R. C., \& Gilliland, R. L. 2004, AJ, 128, 3053

Botticella, M. T., Trundle, C., Pastorello, A., et al. 2010, ApJL, 717, L52

Campbell, H., D'Andrea, C. B., Nichol, R. C., et al. 2013, ApJ, 763, 88

Cardelli, J. A., Clayton, G. C., \& Mathis, J. S. 1989, ApJ, 345, 245

Chomiuk, L., Chornock, R., Soderberg, A. M., et al. 2011, ApJ, 743, 114

Chornock, R., Berger, E., Rest, A., et al. 2013, ApJ, 767, 162

Colina, L., \& Bohlin, R. C. 1994, AJ, 108, 1931

Conley, A., Guy, J., Sullivan, M., et al. 2011, ApJS, 192, 1

Conley, A., Sullivan, M., Hsiao, E. Y., et al. 2008, ApJ, 681, 482

Contreras, C., Hamuy, M., Phillips, M. M., et al. 2010, AJ, 139, 519

Croft, R. A. C., \& Dailey, M. 2011, arXiv:1112.3108

Dressler, A., Bigelow, B., Hare, T., et al. 2011, PASP, 123, 288

Eisenstein, D. J., Zehavi, I., Hogg, D. W., et al. 2005, ApJ, 633, 560

Fabricant, D., Fata, R., Roll, J., et al. 2005, PASP, 117, 1411

Fitzpatrick, E. L. 1999, PASP, 111, 63

Flaugher, B. L., Abbott, T. M. C., Angstadt, R., et al. 2012, Proc. SPIE, 8446, 844611

Folatelli, G., Phillips, M. M., Burns, C. R., et al. 2010, AJ, 139, 120

Foley, R. J., Challis, P. J., Chornock, R., et al. 2013, ApJ, 767, 57

Foley, R. J., Chornock, R., Filippenko, A. V., et al. 2009a, AJ, 138, 376

Foley, R. J., Filippenko, A. V., \& Jha, S. W. 2008, ApJ, 686, 117

Foley, R. J., \& Kasen, D. 2011, ApJ, 729, 55

Foley, R. J., \& Kirshner, R. P. 2013, ApJL, 769, L1

Foley, R. J., Matheson, T., Blondin, S., et al. 2009b, AJ, 137, 3731

Foley, R. J., Papenkova, M. S., Swift, B. J., et al. 2003, PASP, 115, 1220
Foley, R. J., Sanders, N. E., \& Kirshner, R. P. 2011, ApJ, 742, 89 Frieman, J. A., Bassett, B., Becker, A., et al. 2008, AJ, 135, 338 Ganeshalingam, M., Li, W., \& Filippenko, A. V. 2013, MNRAS, 433, 2240

Garg, A., Stubbs, C. W., Challis, P., et al. 2007, AJ, 133, 403

Gezari, S., Chornock, R., Rest, A., et al. 2012, Natur, 485, 217

Gezari, S., Rest, A., Huber, M. E., et al. 2010, ApJL, 720, L77

Glazebrook, K., \& Bland-Hawthorn, J. 2001, PASP, 113, 197

Goldhaber, G., Groom, D. E., Kim, A., et al. 2001, ApJ, 558, 359

Guy, J., Baumont, S., Hardin, D., et al. 2007, A\&A, 466, 11

Guy, J., Sullivan, M., Conley, A., et al. 2010, A\&A, 523, A7

Hamuy, M., Phillips, M. M., Suntzeff, N. B., et al. 1996a, AJ, 112, 2408

Hamuy, M., Phillips, M. M., Suntzeff, N. B., et al. 1996b, AJ, 112, 2391

Hamuy, M., Phillips, M. M., Suntzeff, N. B., et al. 1996c, AJ, 112, 2438

Hayden, B. T., Gupta, R. R., Garnavich, P. M., et al. 2013, ApJ, 764, 191

Hicken, M., Challis, P., Jha, S., et al. 2009a, ApJ, 700, 331

Hicken, M., Challis, P., Kirshner, R. P., et al. 2012, ApJS, 200, 12

Hicken, M., Wood-Vasey, W. M., Blondin, S., et al. 2009b, ApJ, 700, 1097

Hinshaw, G., Larson, D., Komatsu, E., et al. 2013, ApJS, 208, 19

Hodapp, K. W., Kaiser, N., Aussel, H., et al. 2004, AN, 325, 636

Holtzman, J. A., Marriner, J., Kessler, R., et al. 2008, AJ, 136, 2306

Hook, I. M., Jørgensen, I., Allington-Smith, J. R., et al. 2004, PASP, 116, 425

Horne, K. 1986, PASP, 98, 609

Inserra, C., Smartt, S. J., Jerkstrand, A., et al. 2013, ApJ, 770, 128

Jha, S., Kirshner, R. P., Challis, P., et al. 2006, AJ, 131, 527

Jha, S., Riess, A. G., \& Kirshner, R. P. 2007, ApJ, 659, 122

Kaiser, N., Burgett, W., Chambers, K., et al. 2010, Proc. SPIE, 7733, 77330E

Kattner, S., Leonard, D. C., Burns, C. R., et al. 2012, PASP, 124, 114

Kelly, P. L., Hicken, M., Burke, D. L., Mandel, K. S., \& Kirshner, R. P. 2010, ApJ, 715,743

Kelson, D. D. 2003, PASP, 115, 688

Kessler, R., Becker, A. C., Cinabro, D., et al. 2009a, ApJS, 185, 32

Kessler, R., Bernstein, J. P., Cinabro, D., et al. 2009b, PASP, 121, 1028

Kessler, R., Guy, J., Marriner, J., et al. 2013, ApJ, 764, 48

Kirshner, R. P. 2010, in Foundations of Supernova Cosmology, ed. P. RuizLapuente (Cambridge: Cambridge Univ. Press), 151

Krisciunas, K., Phillips, M. M., \& Suntzeff, N. B. 2004, ApJL, 602, L81

Lampeitl, H., Smith, M., Nichol, R. C., et al. 2010, ApJ, 722, 566

Lee, C.-H., Riffeser, A., Koppenhoefer, J., et al. 2012, AJ, 143, 89

Li, W., Leaman, J., Chornock, R., et al. 2011, MNRAS, 412, 1441

Lunnan, R., Chornock, R., Berger, E., et al. 2013, ApJ, 771, 97

Magnier, E. 2006, in in The Advanced Maui Optical and Space Surveillance Technologies Conference, ed. S. Ryan (Maui, HI: Economic Development Board)

Magnier, E. A., Liu, M., Monet, D. G., \& Chambers, K. C. 2008, in IAU Symp. 248, A Giant Step: from Milli- to Micro-arcsecond Astrometry, ed. W. J. Jin, I. Platais, \& M. A. C. Perryman (Cambridge: Cambridge Univ. Press), 553

Mandel, K. S., Narayan, G., \& Kirshner, R. P. 2011, ApJ, 731, 120

Mandel, K. S., Wood-Vasey, W. M., Friedman, A. S., \& Kirshner, R. P. 2009, ApJ, 704, 629 
Marriner, J., Bernstein, J. P., Kessler, R., et al. 2011, ApJ, 740, 72 Marshall, J. L., Burles, S., Thompson, I. B., et al. 2008, Proc. SPIE, 7014, 701454

McCrum, M., Chornock, R., Berger, E., et al. 2014, MNRAS, 437, 656

Miknaitis, G., Pignata, G., Rest, A., et al. 2007, ApJ, 666, 674

Narayan, G., Foley, R. J., Berger, E., et al. 2011, ApJL, 731, L11

Neill, J. D., Hudson, M. J., \& Conley, A. 2007, ApJL, 661, L123

Onaka, P., Tonry, J. L., Isani, S., et al. 2008, Proc. SPIE, 7014, 70140D

Padmanabhan, N., Schlegel, D. J., Finkbeiner, D. P., et al. 2008, ApJ, 674, 1217

Padmanabhan, N., Xu, X., Eisenstein, D. J., et al. 2012, MNRAS, 427, 2132

Pastorello, A., Smartt, S. J., Botticella, M. T., et al. 2010, ApJL, 724, L16

Percival, W. J., Reid, B. A., Eisenstein, D. J., et al. 2010, MNRAS, 401, 2148

Perlmutter, S., Aldering, G., Goldhaber, G., et al. 1999, ApJ, 517, 565

Phillips, M. M. 1993, ApJL, 413, L105

Phillips, M. M., Lira, P., Suntzeff, N. B., et al. 1999, AJ, 118, 1766

Planck Collaboration, Ade, P. A. R., Aghanim, N., et al. 2013, arXiv:1303.5076

Rau, A., Kulkarni, S. R., Law, N. M., et al. 2009, PASP, 121, 1334

Rest, A., Stubbs, C., Becker, A. C., et al. 2005, ApJ, 634, 1103

Riess, A. G., Filippenko, A. V., Challis, P., et al. 1998, AJ, 116, 1009

Riess, A. G., Kirshner, R. P., Schmidt, B. P., et al. 1999, AJ, 117, 707

Riess, A. G., Macri, L., Casertano, S., et al. 2011, ApJ, 730, 119

Riess, A. G., Press, W. H., \& Kirshner, R. P. 1996, ApJ, 473, 88

Sako, M., Bassett, B., Connolly, B., et al. 2011, ApJ, 738, 162

Sanders, N. E., Soderberg, A. M., Foley, R. J., et al. 2013, ApJ, 769, 39

Sanders, N. E., Soderberg, A. M., Valenti, S., et al. 2012, ApJ, 756, 184

Schechter, P. L., Mateo, M., \& Saha, A. 1993, PASP, 105, 1342
Schlafly, E. F., \& Finkbeiner, D. P. 2011, ApJ, 737, 103

Schlafly, E. F., Finkbeiner, D. P., Juric, M., et al. 2012, ApJ, 756, 158

Schlegel, D. J., Finkbeiner, D. P., \& Davis, M. 1998, ApJ, 500, 525

Schmidt, G. D., Weymann, R. J., \& Foltz, C. B. 1989, PASP, 101, 713

Scolnic, D. M., Rest, A., Riess, A., et al. 2014b, ApJ, 795, 45

Scolnic, D. M., Riess, A. G., Foley, R. J., et al. 2014a, ApJ, 780, 37

Silverman, J. M., Foley, R. J., Filippenko, A. V., et al. 2012a, MNRAS, 425,1789

Silverman, J. M., Ganeshalingam, M., Li, W., \& Filippenko, A. V. 2012b, MNRAS, 425, 1889

Stetson, P. B. 1987, PASP, 99, 191

Stritzinger, M. D., Phillips, M. M., Boldt, L. N., et al. 2011, AJ, 142, 156

Stubbs, C. W., Doherty, P., Cramer, C., et al. 2010, ApJS, 191, 376

Stubbs, C. W., \& Tonry, J. L. 2006, ApJ, 646, 1436

Sullivan, M., Conley, A., Howell, D. A., et al. 2010, MNRAS, 406, 782

Sullivan, M., Guy, J., Conley, A., et al. 2011, ApJ, 737, 102

Taylor, J. 1997, Introduction to Error Analysis (2nd ed.; Mill Valley, CA: University Science Books)

Tonry, J. L., Stubbs, C. W., Kilic, M., et al. 2012a, ApJ, 745, 42

Tonry, J. L., Stubbs, C. W., Lykke, K. R., et al. 2012b, ApJ, 750, 99

Tripp, R. 1998, A\&A, 331, 815

Valenti, S., Taubenberger, S., Pastorello, A., et al. 2012, ApJL, 749, L28

Wade, R. A., \& Horne, K. 1988, ApJ, 324, 411

Wang, X., Filippenko, A. V., Ganeshalingam, M., et al. 2009, ApJL, 699, L139

Wood-Vasey, W. M., Friedman, A. S., Bloom, J. S., et al. 2008, ApJ, 689, 377

Wood-Vasey, W. M., Miknaitis, G., Stubbs, C. W., et al. 2007, ApJ, 666, 694 